\title{
A Single-Step Asymmetric Phosphodiester Synthesis from Alcohols with Phosphoenolpyruvate Phosphodiester
}

\author{
Kohei Fujiyoshi ${ }^{\mathrm{a}}$ \\ Shigehiro A. Kawashima ${ }^{a}$ \\ Kenzo Yamatsugu*a \\ Motomu Kanai*a \\ ${ }^{a}$ Graduate School of Pharmaceutical Sciences, The University \\ of Tokyo, Hongo, Bunkyo-ku, Tokyo 113-0033, Japan \\ yamatsugu@mol.f.u-tokyo.ac.jp \\ kanai@mol.f.u-tokyo.ac.jp
}
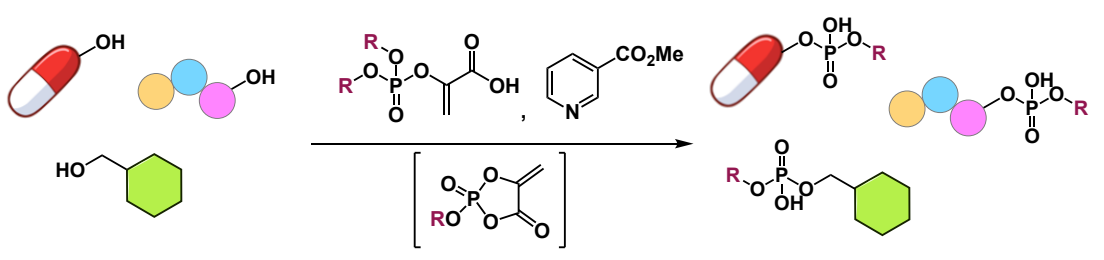

Single-step phosphodiester synthesis Simple operation. Eunctional group-tolerant.
Abstract Phosphodiesters are important structural motifs observed in a diverse field of molecular science. It is, thus, important to develop a simple and robust way to synthesize them from corresponding alcohols. Here we report a single-step asymmetric phosphodiester synthesis from alcohols with phosphoenolpyruvate phosphodiesters as phosphoryl donors. This transformation allows for the use of various functionalized alcohols as substrates, and would be useful for diverse fields including biology and medicine.

Key words Phosphorylation, alcohol, phosphodiester, phosphoenolpyruvate, pentacoordinate phosphorous

Phosphate esters play pivotal roles in the life science. ${ }^{1}$ They are divided into three classes; phosphomonoester, phosphodiester, and phosphotriester, each of which prevails among the molecules of life and shows distinct functions. ${ }^{2}$ Phosphodiesters are, especially, fundamental motifs observed in biomolecules. For example, DNA, which is a chain of deoxyribonucleic acids connected through phosphodiester linkages, encodes the genetic information, and a phospholipid, which has two hydrophobic fatty acid chains and one polar phosphodiester head group, separates the interior of the cell from the outside environment. Inspired by those examples in life, a lot of functional molecules containing phosphodiesters are designed and used for various purposes, such as a methyl phosphate as an abiotic lipid analog for liposome-mediated drug delivery system, ${ }^{3}$ a "PC-inverse" choline phosphate designed to strongly interact with natural phosphatidylcholine in the plasma membrane, ${ }^{4}$ lower alkyl phosphates as enzyme inhibitors through transition-state mimicry ${ }^{5}$ or increasing binding affinity to a target enzyme, ${ }^{6}$ a methyl phosphate metabolically stabilizing a nucleoside analog, 7 and lower alkyl phosphate prodrugs to increase water solubility of molecules (Figure 1). ${ }^{8}$

A straightforward way to access phosphodiesters is via phosphorylation of alcohol starting materials. There are mainly

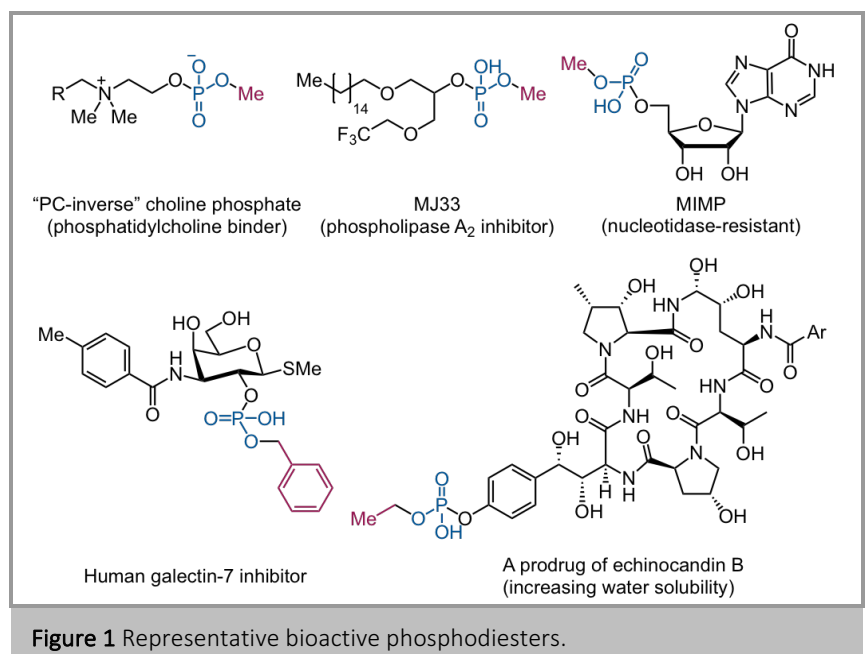

two ways to synthesizing asymmetric phosphodiesters from alcohols: (1) the formation of trivalent phosphite followed by oxidation, and (2) the reaction with a pentavalent phosphoryl donor (Scheme 1a,b). In a typical example of the first approach, two different alcohols are sequentially reacted with a trivalent chlorophosphoramidite reagent, the product of which is then oxidized to the protected pentavalent phosphate triester. ${ }^{9}$ Finally, the removal of the protecting group under acidic, reductive, or basic conditions (PG = alkyl, benzyl, or cyanoethyl, respectively) produces the desired phosphodiester. This method is quite robust, but requires four steps and is sometimes incompatible with substrates, which are sensitive to oxidation. In a typical example of the second approach, phosphoryl chloride and its derivatives are used as phosphoryl donors (Scheme 1b). ${ }^{10}$ Although reactions of two different alcohols and water with phosphoryl chloride can directly provide phosphodiesters, it is often difficult to control the reaction, resulting in complex mixtures with various substitution patterns. Although several masked phosphoryl donors with attenuated reactivity were developed to circumvent this issue, ${ }^{11}$ multiple steps are still 
required to synthesize asymmetric phosphodiesters. Thus, an alternative method for phosphodiester synthesis with a simple operation and with high functional group tolerance is still in high demand. Here we report a phosphodiester synthesis from alcohols using phosphoenolpyruvate phosphodiesters as phosphoryl donors. This transformation provides asymmetric phosphodiesters from various functionalized alcohols in a singlestep reaction.

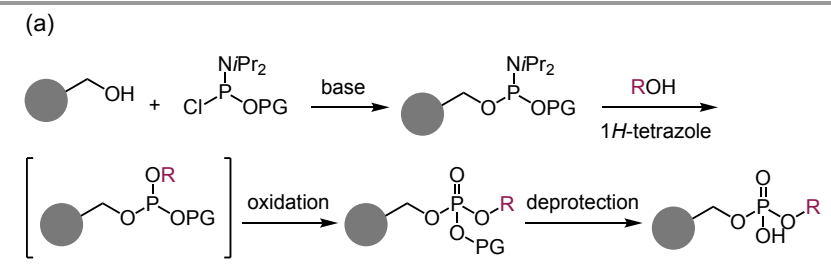

(b)

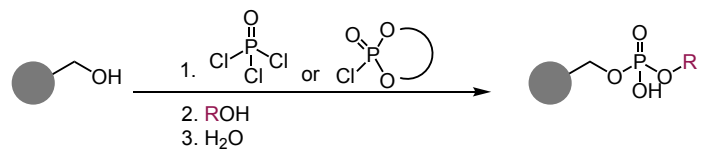

(c) Our previous work

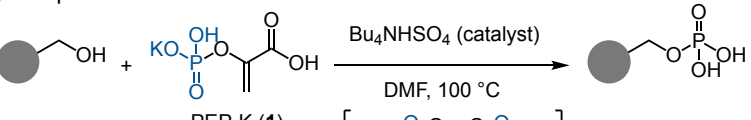

$$
\begin{aligned}
& \text { PEP-K (1) }
\end{aligned}
$$

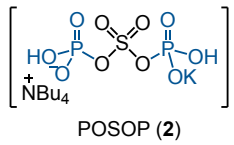

(d) Hypothesis

$$
\mathrm{CH}_{+}^{\text {(d) Hypothesis }}
$$

(e) Initial findings

$$
\begin{aligned}
& \text { (4:5equiv.) } \\
& \begin{array}{ccccc:c}
\hline \text { Entry } & \begin{array}{c}
\text { Phosphoryl } \\
\text { donor }
\end{array} & \mathrm{X} & \text { Yield of 7 (\%) } & \text { Yield of 8 (\%) } \\
\hline 1 & 1 & 30 & - & 88 & 0 \\
2 & 1 & 0 & - & 7 & \\
3 & 6 & 30 & 59 & 23 & \\
4 & 6 & 0 & \text { ND } & 25 \\
\hline
\end{array}
\end{aligned}
$$

Scheme 1 Phosphate ester synthesis from alcohols. ${ }^{a} A$ representative $\mathrm{P}(\mathrm{III})$ chemistry for phosphodiester synthesis. ${ }^{b} A$ representative $P(V)$ chemistry for

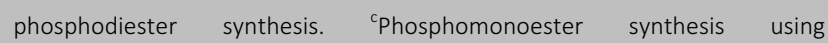
phosphoenolpyruvate monopotassium salt (1) and tetrabutylammonium hydrogen sulfate (ref. 12). ${ }^{\text {II }}$ itial hypothesis for phosphodiester synthesis with phosphoenolpyruvate phosphomonoester (3). EInitial findings in phosphodiester synthesis using monomethyl phorphoenolpyruvate sodium salt (6). ND denotes not detected.

We previously reported a catalytic chemoselective phosphorylation of alcohols affording phosphomonoesters in a single step with tetrabutylammonium hydrogen sulfate (TBAHS) and phosphoenolpyruvate monopotassium salt (PEP-K: 1) as a catalyst and a phosphoryl donor, respectively (Scheme 1c and 1e, entry 1).12 In this reaction, a mixed anhydride species, POSOP (2), which is catalytically generated from two molecules of phosphoenolpyruvate and one molecule of hydrogen sulfate ion, serves as a reactive phosphorylation species. We hypothesized that if we use phosphoenolpyruvate phosphomonoester (3) as a starting phosphoryl donor, an analogous phosphorylation species (4) would be formed, affording an asymmetric phosphodiester (Scheme 1d).

Based on this hypothesis, we reacted 3-phenyl-1-propanol (5) with monomethyl phosphoenolpyruvate sodium salt (6) ${ }^{13}$ in the presence of $30 \mathrm{~mol} \%$ of TBAHS in DMF at $100{ }^{\circ} \mathrm{C}$ for 5 hours (Scheme 1e). On the contrary to our hypothesis, however, phosphodiester 7 was produced in 59\% yield concomitant with phosphomonoester 8 obtained in 23\% yield (entry 3). Intriguingly, the formation of phosphomonoester $\mathbf{8}$ was not TBAHS-dependent, and $\mathbf{8}$ was obtained in 25\% yield by simply reacting substrate 5 with phosphoryl donor 6 at $100{ }^{\circ} \mathrm{C}$ in the absence of TBAHS (Scheme 1e, entry 4). Since a brief optimization of the reaction conditions of entry 3 for suppressing the generation of $\mathbf{8}$ did not result in success, and PEP-K (1) afforded the phosphomonoester $\mathbf{8}$ only in $7 \%$ yield in the absence of TBAHS on the contrary to 6 (entry 2 vs. entry 4), we turned our attention to this interesting catalyst-independent high reactivity of 6 and decided to explore the reactivity of phosphoenolpyruvate phophoesters.

Clark and Kirby reported that phosphoenolpyruvate phosphoesters were hydrolyzed rapidly through an intermediate of cyclic acyl phosphate (e.g., 9 in Scheme 1e), which is generated via the intramolecular nucleophilic attack of the carboxylic acid moiety to the phosphorous atom followed by the elimination of the alcohol. ${ }^{14}$ The formation of phosphomonoester 8 with 6 in Scheme 1e could be explained by the attack of the substrate hydroxy group to the cyclic acyl phosphate $\mathbf{9}$ followed by elimination of the enol oxygen and methanolysis of the resulting anhydride. Higher reactivity of $\mathbf{6}$ than $\mathbf{1}$ in the absence of TBAHS (i.e., Scheme 1e, entry 2 vs. entry 4 ) was likely due to smaller negative charge at the phosphate group, thus facilitating the formation of putative intermediate 9. Based on this idea, we envisioned that phosphoenolpyruvate phosphodiester would serve as a phosphoryl donor for the synthesis of asymmetric phosphodiesters of various alcohols.

First, we reacted $\mathbf{5}$ with three equivalents of dimethyl phosphoenolpyruvate (10), which was prepared just before the phosphorylation reaction with Perkow reaction using bromopyruvic acid and trimethyl phosphite, in acetonitrile at $60{ }^{\circ} \mathrm{C}$ for 12 hours without any additive (Table 1 , entry 1 ). The substrate $\mathbf{5}$ was nearly consumed, and methyl phosphate $\mathbf{7}$ was obtained in $9 \%$ yield as we expected. Since the product phosphate diester could be unstable in the protonated state under acidic conditions on heating, we examined addition of one equivalent of basic additives to neutralize the product phosphate in the reaction (entries 2-4). Among examined, pyridine worked the best to afford 7 in $67 \%$ yield (entry 4). Next, we examined several substituted pyridine derivatives (entries 5-7), and found that methyl nicotinate (11) increased the yield and 7 was obtained in $73 \%$ yield (entry 7 ). The methoxycarbonyl group at 
the 3-position of the pyridine may attenuate nucleophilicity to suppress undesired reactions while maintaining its ability to neutralize the product in the reaction. The reaction completed within 6 hours to afford 7 in $72 \%$ yield (entry 8). Neither increasing the amount of phosphoryl donor 10 to 4.5 equivalents (entry 9) nor raising temperature to $80^{\circ} \mathrm{C}$ (entry 10) improved the yield, while conducting the reaction at room temperature significantly decreased the yield and the most of the substrate was recovered unchanged (entry 11).

Table 1 Optimization of the Reaction Conditions

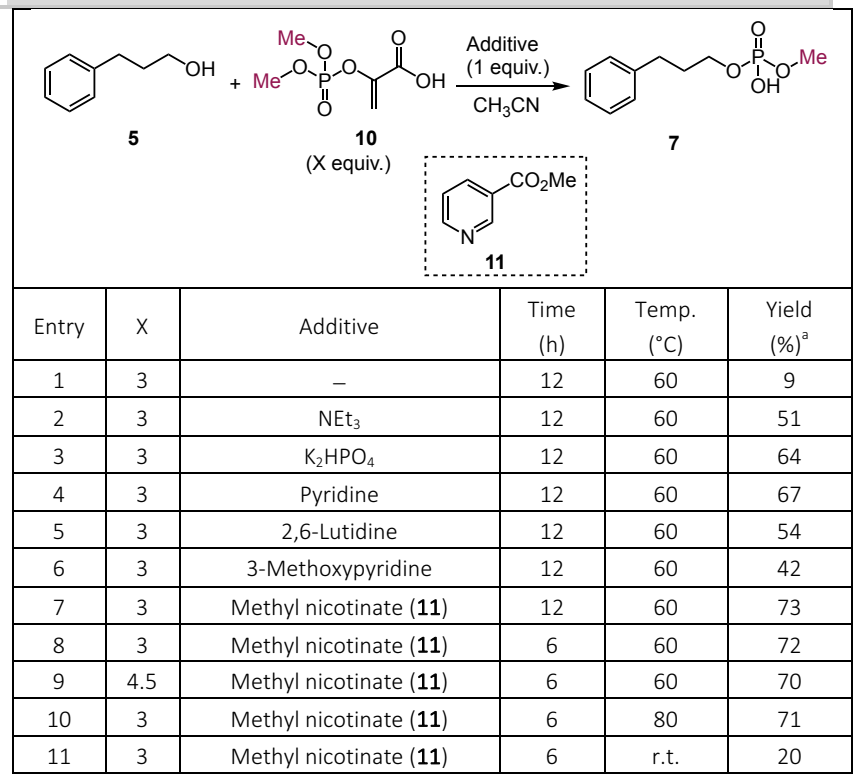

${ }^{a}$ Determined by ${ }^{1} \mathrm{H}$ NMR

Having identified the optimized reaction conditions, we next investigated the substrate scope of this transformation. As shown in Scheme 2, a secondary alcohol is a competent substrate and underwent the phosphorylation to afford 12 in $49 \%$ yield. Substrates with a range of functional groups including fluoro(15: y. 38\%), chloro- (16, y. 45\%), bromo- (13: y. 42\%, 17: y. 40\%), alkoxy (14: y. 46\%, 18: 37\%), nitro (19: y. 34\%), and thiomethyl (20: y. 47\%)-substituted phenyl groups afforded the product phosphodiesters in moderate yields. It was noteworthy that the phosphodiester $\mathbf{2 0}$ was directly obtained considering that the thiomethyl group is prone to oxidation in a phophoramidite chemistry (Scheme 1a). The reaction conditions were mild, and styrene byproducts derived from the $\beta$ elimination of the product phosphates were not observed in the transformation to 15-20. An aldehyde (21: y. 48\%), a carboxylic acid (22: y. 49\%), an ester (23: y. 52\%) and a ketone (24: 49\%) were accommodated in this transformation. A chemoselective phosphorylation of an alcohol in the presence of a phenol was possible, and $\mathbf{2 5}$ was obtained in $32 \%$ yield, where we did not observe phenol-phosphorylated products in the crude mixture. An acid-labile glycosidic linkage was tolerated, and a phosphorylated carbohydrate (26) was obtained in 30\% yield. Interestingly, when a glucose derivative with its 4,6-hydroxy groups unprotected, was used as a substrate, cyclic phosphate (27: y. 37\%) was obtained, which could be generated via first methyl phosphate formation either on 4- or 6-hydroxy group followed by an intramolecular attack of the remaining hydroxy group to the methyl phosphate. Amino acid serine (28: y. 45\%) and threonine (29: y. 41\%) were also good substrates for this transformation. Finally, synthesis of phosphate esters other than methyl were possible, and ethyl (30: y. 49\%) and benzyl (31: y. $43 \%$ ) phosphates were obtained in a single step reaction from an alcohol starting material (For the preparation of diethyl/dibenzyl phosphoenolpyruvate, see Supporting Information). Although the yield was moderate in most of the cases due to concomitant formation of small amounts of several unidentified byproducts, which could be mostly removed through the acid-base extraction (see Supporting Information), the concise operation to directly access phosphodiesters is an advantage over existing methods (e.g., Scheme 1a,1b).

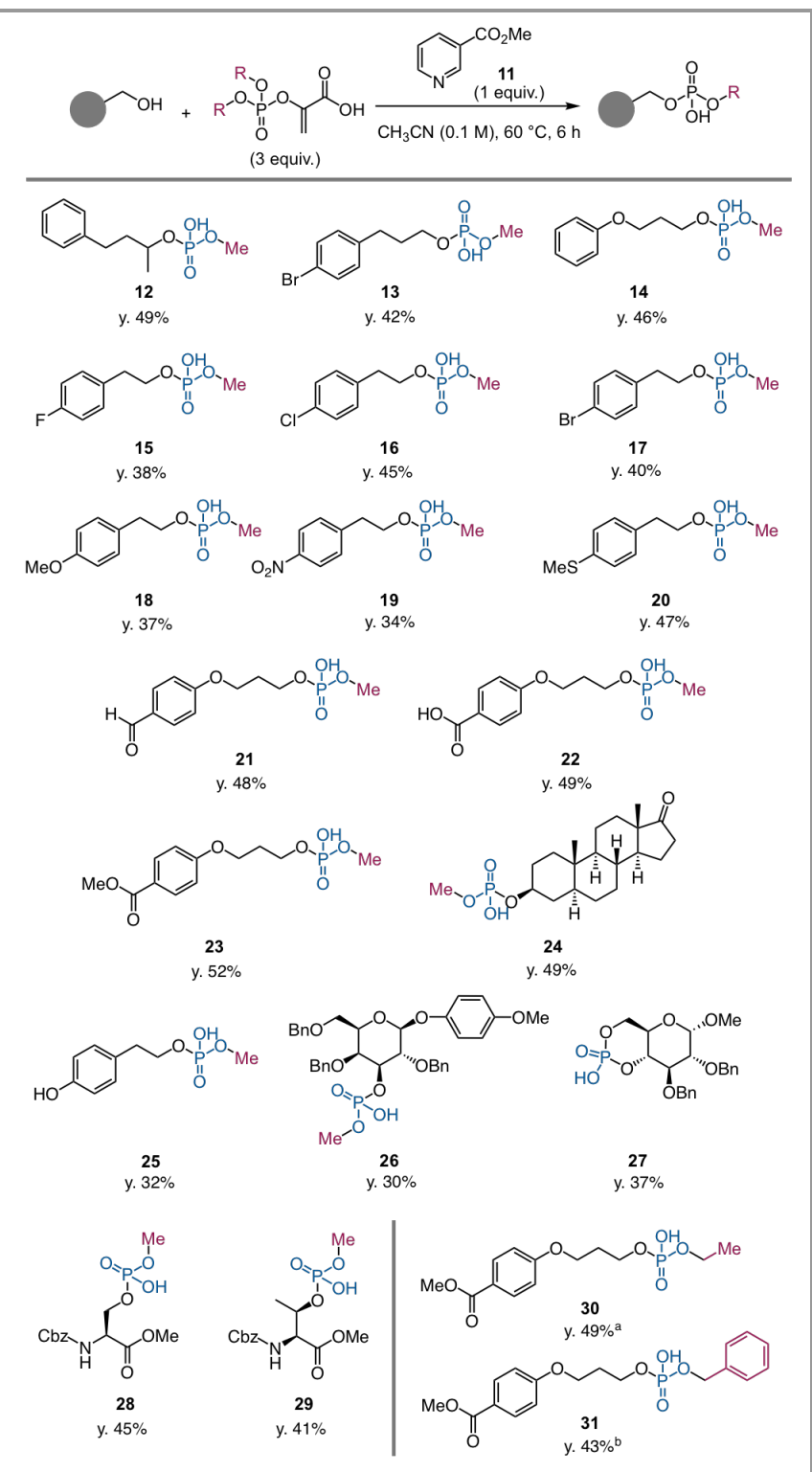

Scheme 2 Substrate scopes. The yields are isolated yield. ${ }^{\text {a }} 6$ equivalents of the phosphoryl donor, 2 equivalents of methyl nicotinate, $80{ }^{\circ} \mathrm{C}, 13$ hours. ${ }^{b} 13$ hours.

To get insights into the reaction mechanisms, we did several investigations. First, we conducted a reaction using a carboxy ester derivative of phosphoenolpyruvate phosphodiester as a phosphoryl donor. When $\mathbf{5}$ was treated with 3 equivalents of phosphoenolpyruvate triester $\mathbf{3 2}$ and one equivalent of methyl 
nicotinate (11) under the optimized conditions, phosphorylation product $\mathbf{7}$ was not obtained at all, and the starting material $\mathbf{5}$ and 32 were recovered (Scheme $3 a$ ). This result is consistent with our idea that cyclic acyl phosphate, which results from intramolecular attack of the carboxylic acid moiety to the phosphorous atom of phosphoenolpyruvate phosphodiester, is an actual phosphorylation species. Second, to detect reaction intermediates, we conducted mass spectrometry analysis of the reaction mixture. When we analyzed the reaction mixture of alcohol $\mathbf{5}$ and dimethyl phosphoenolpyruvate $\mathbf{1 0}$ before the completion of the reaction (15 $\mathrm{min}$ ) with electrospray ionization mass spectrometry (ESI-MS), we observed a species of $m / z 301.1$, which corresponds to the adduct of alcohol $\mathbf{5}$ to the cyclic acyl phosphate generated from 10, such as $\mathbf{3 3}$ (Scheme $3 \mathrm{~b}$ and Figure S1). This result also indicates that the cyclic acyl phosphate is the phosphorylating species.

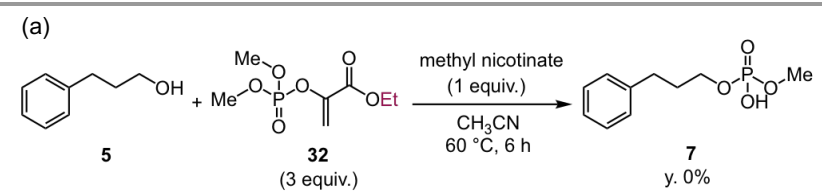

(b)

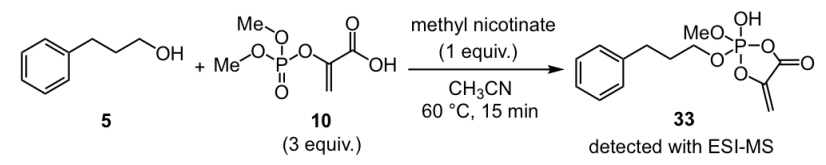

Scheme 3 Investigations of the Reaction Mechanisms. ${ }^{a} A$ control reaction using carboxy-ethylated phosphoryl donor $32 .{ }^{b}$ Detection of a reaction intermediate with ESI-MS

Based on these results, a proposed reaction mechanism is shown in Scheme 4. Phosphoenolpyruvate phosphodiester $\mathbf{3 4}$ is in equilibrium with cyclized pentacoordinate phosphorous species 35. ${ }^{15}$ After pseudorotation to place the alkoxy group into an apical position (36), one of the alcohol group ( $\mathrm{ROH}$ ) is released to generate a reactive cyclic acyl phosphate $\mathbf{3 7}$ in a reversible manner. ${ }^{14}$ The substrate alcohol $\left(\mathrm{R}^{\prime} \mathrm{OH}\right)$, then, attacks 37 to generate another pentacoordinate phosphorous species $\mathbf{3 8 . 1 6}$ These elimination and addition of alcohols are all reversible. When 38 undergoes pseudorotation to place the enol oxygen into the apical position (39), the enol group can be irreversibly eliminated to generate acyclic acyl phosphate 40. Acyclic acyl phosphate are known to prefer nucleophilic attack at the carbonyl carbon to at the phosphorous atom, serving as a good acylating agent. ${ }^{17}$ Thus, $\mathbf{4 0}$ reacts with the leaving group alcohol (ROH) to generate the protonated from of phosphodiester (42) and pyruvate ester of the alcohol (41). Methyl nicotinate stabilizes the product phosphodiester $\mathbf{4 2}$ by neutralizing to generate pyridinium phosphate salt $\mathbf{4 3}$ in the reaction mixture at $60^{\circ} \mathrm{C}$, which in turn provides protonated 42 after acidic work-up.

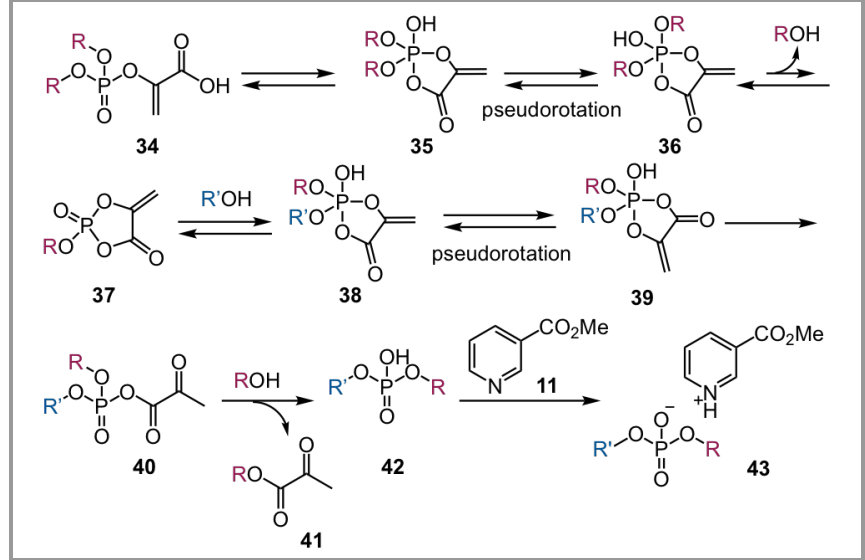

Scheme $4 \mathrm{~A}$ proposed mechanism of the phosphodiester synthesis with phosphoenolpyruvate phosphodiester

In conclusion, we developed a single step asymmetric phosphodiester synthesis from alcohols and phosphoenolpyruvate phosphodiesters. This reaction provides a variety of functionalized asymmetric phosphodiesters in a short and concise procedure, which would be useful in diverse field including biology and medicine.

\section{Funding Information}

This work was supported from JSPS KAKENHI Grant Number JP17K15420 (K.Y.), and JP20H00489 (M.K.), and SUNBOR Grant (K.Y.).

\section{References and Notes}

(1) Hunter, T. Philos. Trans. R. Soc. B 2012, 367, 2513.

(2) Kirby, A. J.; Nome, F. Acc. Chem. Res. 2015, 48, 1806.

(3) Oumzil, K.; Benizri, S.; Tonelli, G.; Staedel, C.; Appavoo, A.; Chaffanet, M.; Navailles, L.; Barthélémy, P. ChemMedChem 2015, 10, 1797.

(4) Yu, X.; Liu, Z.; Janzen, J.; Chafeeva, I.; Horte, S.; Chen, W.; Kainthan, R. K.; Kizhakkedathu, J. N.; Brooks, D. E. Nat. Mater. 2012, 11, 468.

(5) Jain, M. K.; Tao, W.; Rogers, J.; Arenson, C.; Eibl, H.; Yu, B. Z. Biochemistry 1991, 30, 10256.

(6) Masuyer, G.; Jabeen, T.; Öberg, C. T.; Leffler, H.; Nilsson, U. J.; Acharya, K. R. FEBS J. 2012, 279, 193.

(7) Sosa, M.; Saha, A.; Giner - Sorolla, A.; Hadden, E.; Hadden, J. W. Ann. N. Y. Acad. Sci. 1993, 685, 458.

(8) Rodriguez, M. J.; Vasudevan, V.; Jamison, J. A.; Borromeo, P. S.; Turner, W. W. Bioorg. Med. Chem. Lett. 1999, 9, 1863.

(9) Marugg, J. E.; Burik, A.; Tromp, M.; van der Marel, G. A.; van Boom, J. H. Tetrahedron Lett. 1986, 27, 2271.

(10) Bonnet, H. Justus Liebigs Ann. Chem. 1857, 104, 337.

(11) Mushika, Y.; Hata, T.; Mukaiyama, T. Bull. Chem. Soc.Jpn. 1971, 44, 232; Ramirez, F.; Glaser, S. L.; Stern, P.; Ugi, I.; Lemmen, P. Tetrahedron 1973, 29, 3741; Ramirez, F.; Marecek, J. F.; Okazaki, H. J. Am. Chem. Soc. 1976, 98, 5310.

(12) Domon, K.; Puripat, M.; Fujiyoshi, K.; Hatanaka, M.; Kawashima, S. A.; Yamatsugu, K.; Kanai, M. ACS Cent. Sci. 2020, 6, 283.

(13) Hamasaki, N.; Kawamura, H.; Ohtsu, N.; Nakakoshi, I.; Ataka, K.; Oomori, K.; Kouno, M. Preparation of monosodium phosphoenolpyruvate and its use as a visceral function improver, especially for treatment of ischemia. EP239357A2, 1987.

(14) Clark, V. M.; Kirby, A. J. J. Am. Chem. Soc. 1963, 85, 3705.

(15) Benkovic, S. J.; Schray, K. J. J. Am. Chem. Soc. 1969, 91, 5653.

(16) Baccolini, G.; Boga, C.; Micheletti, G. Phosphorus, Sulfur, Silicon Relat. Elem. 2010, 185, 2303. 
Supporting Information for

\title{
A Single-Step Asymmetric Phosphodiester Synthesis from Alcohols with Phosphoenolpyruvate Phosphodiester
}

\author{
K. Fujiyoshi, S. A. Kawashima, K. Yamatsugu*, and M. Kanai*
}

Correspondence to: kanai@mol.f.u-tokyo.ac.jp (M.K.), yamatsugu@mol.f.u-tokyo.ac.jp (K.Y.) 


\section{Table of contents}

\section{Materials and Methods}

Preparation of dimethyl phosphoenolpyruvate

General procedures for phosphorylation of alcohols

Reaction of 32 with 3-phenyl-1-propanol

Detection of 33 with LC-MS.

Characterization of phosphorylated products

References 


\section{Materials and Methods}

\section{General}

NMR spectra were recorded on JEOL ECS400 (400 MHz for ${ }^{1} \mathrm{H}$ NMR, $100 \mathrm{MHz}$ for ${ }^{13} \mathrm{C} \mathrm{NMR}$ and $159 \mathrm{MHz}$ for ${ }^{31} \mathrm{P}$ NMR) spectrometers. Chemical shifts were reported in ppm on the $\delta$ scale relative to residual $\mathrm{CHCl}_{3}(\delta$ $=7.24$ for ${ }^{1} \mathrm{H}$ NMR and $\delta=77.0$ for $\left.{ }^{13} \mathrm{C} \mathrm{NMR}\right), \mathrm{CHD}_{2} \mathrm{OD}\left(\delta=3.31\right.$ for ${ }^{1} \mathrm{H} \mathrm{NMR}$ and $\delta=49.0$ for $\left.{ }^{13} \mathrm{C} \mathrm{NMR}\right)$, or $\mathrm{CHD}_{2} \mathrm{CN}\left(\delta=1.94\right.$ for ${ }^{1} \mathrm{H}$ NMR and $\delta=118.2$ for $\left.{ }^{13} \mathrm{C} \mathrm{NMR}\right)$ as an internal reference, and $85 \% \mathrm{H}_{3} \mathrm{PO}_{4}$ aq. $(\delta=$ 0 for $\left.{ }^{31} \mathrm{P} \mathrm{NMR}\right)$ as an external reference, respectively.

Preparative HPLC was conducted by using a JASCO HPLC system equipped with a UV-2075 spectrometer, PU4086 pumps, a DG-4580 degasser, and an MX-2080-32 mixer, and a CO-4065 column oven. General eluent was linear gradient of MeCN in 0.1\% TFA (LC-MS grade) aq. (10\% acetonitrile for 5 min, followed by a linear gradient of 10-100\% acetonitrile over $60 \mathrm{~min}$. YMC-Triart C18, $254 \mathrm{~nm}$ ), unless otherwise stated. C18 reverse phase column was used at $40{ }^{\circ} \mathrm{C}$, unless otherwise stated.

MQ means distilled water purified with a Millipore Milli-Q water purification system (Merck K. Ga. Co., Darmstadt, Germany).

LC-MS analysis was conducted by using an Agilent Technologies LC-MS (ESI) system equipped with a 1260 Infinity High Performance Degasser, an Agilent 1260 Infinity Binary Pump, a 1260 Infinity Standard Autosampler, a 1290 Infinity Thermostatted Column Compartment, a 1260 Infinity Variable Wavelength Detector, and an Agilent 6120 Single Quadrupole LC-MS or Shimadzu LCMS-2020. Retention times (Rt/min) were recorded using a gradient elution method of $2-90 \% \mathrm{~B}$ over $9 \mathrm{~min}$, where solution A consisted of water (buffered with $0.1 \% \mathrm{HCO}_{2} \mathrm{H}$ ) and solution $\mathrm{B}$ consisted of acetonitrile (LC-MS grade) unless otherwise stated. C18 reverse phase column $(2.0 \times 50 \mathrm{~mm}$; YMC-Triart C18; YMC Co., Ltd. $)$ was used at $40{ }^{\circ} \mathrm{C}$ at a flow rate of $0.2 \mathrm{~mL} / \mathrm{min}$. The eluent was monitored by absorbance at $230 \mathrm{~nm}$, unless otherwise stated.

Reactions were carried out in dry solvents under an argon atmosphere, unless otherwise stated. Other reagents were used as received from commercial sources (Aldrich, TCI, or FUJIFILM Wako), unless otherwise stated. 


\section{Preparation of dimethyl phosphoenolpyruvate (10)}

Dimethyl phosphoenolpyruvate was prepared according to the literature. ${ }^{1}$ A heat-gun dried and argon-flushed test tube equipped with a magnetic stirrer bar was charged with 3-bromopyruvic acid (1.0 equiv.) and diethyl ether $(1.66 \mathrm{M})$. Then, a solution of trimethyl phosphite (1.0 equiv.) in diethyl ether $(1.66 \mathrm{M})$ was added dropwise at a gently reflux rate, and the reaction mixture was stirred at r.t. After $1 \mathrm{~h}$, the solvent was removed under high vacuum, and the resulting dimethyl phosphoenolpyruvate was used without further purification.

\section{General procedures for phosphorylation of alcohols \\ Procedure S (Standard)}

To the freshly-prepared dimethyl phosphoenolpyruvate (300 $\mu$ mol, 3.0 equiv.), were added sequentially acetonitrile $(0.10 \mathrm{M})$, substrate $(100 \mu \mathrm{mol}, 1.0$ equiv.), and methyl nicotinate (13.7 mg, $100 \mu \mathrm{mol}, 1.0$ equiv.). The mixture was warmed to $60{ }^{\circ} \mathrm{C}$ and stirred for $6 \mathrm{~h}$. Then the reaction mixture was cooled to r.t. and concentrated. After adding saturated $\mathrm{Na}_{2} \mathrm{CO}_{3}$ aq., the mixture was washed three times with diethyl ether. The aqueous layer was acidified with concentrated $\mathrm{HCl}$ to $\mathrm{pH} 1$, and extracted five times with dichloromethane. The combined organic layers were dried over with $\mathrm{Na}_{2} \mathrm{SO}_{4}$, filtered and concentrated to afford crude product, which was purified by preparative HPLC to afford the corresponding phosphorylated product.

\section{Procedure A}

To the freshly-prepared dimethyl phosphoenolpyruvate (300 $\mu$ mol, 3.0 equiv.), were added sequentially acetonitrile $(1.0 \mathrm{~mL}, 0.10 \mathrm{M})$, substrate $(100 \mu \mathrm{mol}, 1.0$ equiv.), and methyl nicotinate $(13.7 \mathrm{mg}, 100 \mu \mathrm{mol}, 1.0$ equiv.). The mixture was warmed to $60{ }^{\circ} \mathrm{C}$ and stirred for $6 \mathrm{~h}$. Then the reaction mixture was cooled to r.t. and concentrated. After adding $1 \mathrm{~N} \mathrm{HCl}$, the mixture was extracted five times with dichloromethane. The combined organic layers were dried over with $\mathrm{Na}_{2} \mathrm{SO}_{4}$, filtered and concentrated to afford crude product, which was purified by preparative HPLC to afford the corresponding phosphorylated product.

\section{Procedure B}

To the freshly-prepared dimethyl phosphoenolpyruvate (3.0 equiv.), were added sequentially acetonitrile (1.0 $\mathrm{mL}, 0.10 \mathrm{M}$ ), substrate (100 $\mathrm{mol}, 1.0$ equiv.), and methyl nicotinate (13.7 mg, $100 \mu \mathrm{mol}, 1.0$ equiv.). The mixture was warmed to $60{ }^{\circ} \mathrm{C}$ and stirred for $6 \mathrm{~h}$. Then the reaction mixture was cooled to r.t., concentrated and purified by preparative HPLC to give the corresponding phosphorylated product.

\section{Procedure C}

A heat-gun dried and argon-flushed test tube equipped with a magnetic stirrer bar was charged with 3bromopyruvic acid (600 $\mu \mathrm{mol}, 6.0$ equiv.) and diethyl ether $(1.66 \mathrm{M})$. Then, a solution of the triethyl phosphite (600 $\mu \mathrm{mol}, 6.0$ equiv.) in diethyl ether $(1.66 \mathrm{M})$ was added dropwise at a gently reflux rate, and the reaction mixture was stirred at r.t. for $2 \mathrm{~h}$. The solvent was removed under high vacuum, and the resulting diethyl 
phosphoenolpyruvate was used without further purification.

To the freshly-prepared diethyl phosphoenolpyruvate (6.0 equiv.), were added sequentially acetonitrile ( $1.0 \mathrm{~mL}$, $0.10 \mathrm{M})$, substrate ( $100 \mu \mathrm{mol}, 1.0$ equiv.), and methyl nicotinate (200 $\mu \mathrm{mol}, 2.0$ equiv.). The mixture was warmed to $80^{\circ} \mathrm{C}$ and stirred for $13 \mathrm{~h}$. Then the reaction mixture was cooled to r.t. and concentrated. After adding saturated $\mathrm{Na}_{2} \mathrm{CO}_{3}$ aq, the mixture was washed three times with diethyl ether. The aqueous layer was acidified with concentrated $\mathrm{HCl}$ to $\mathrm{pH} \mathrm{1}$, and extracted five times with dichloromethane. The combined organic layers were dried over $\mathrm{Na}_{2} \mathrm{SO}_{4}$, filtered and concentrated to afford crude product, which was purified by preparative HPLC to afford the corresponding phosphorylated product.

\section{Procedure D}

A heat-gun dried and argon-flushed test tube equipped with a magnetic stirrer bar was charged with 3bromopyruvic acid (3.0 equiv.) and diethyl ether (1.66M). Then, a solution of the tribenzyl phosphite (3.0 equiv.) in diethyl ether $(1.66 \mathrm{M})$ was added dropwise at a gently reflux rate, and the reaction mixture was stirred at r.t. for $2 \mathrm{~h}$. The solvent was removed under high vacuum, and the resulting residue was washed with anhydrous hexane three times. Remained hexane was removed under high vacuum, and the resulting dibenzyl phosphoenolpyruvate was used without further purification.

To the freshly-prepared dibenzyl phosphoenolpyruvate (3.0 equiv.), were added sequentially acetonitrile (1.0 $\mathrm{mL}, 0.10 \mathrm{M}$ ), substrate (100 $\mu \mathrm{mol}, 1.0$ equiv.), and methyl nicotinate (13.7 mg, $100 \mu \mathrm{mol}, 1.0$ equiv.). The mixture was warmed to $60{ }^{\circ} \mathrm{C}$ and stirred for $13 \mathrm{~h}$. Then the reaction mixture was cooled to r.t. and concentrated. After adding saturated $\mathrm{Na}_{2} \mathrm{CO}_{3}$ aq, the reaction mixture was washed three times with diethyl ether. The aqueous layer was acidified with concentrated $\mathrm{HCl}$ to $\mathrm{pH} 1$, and extracted five times with dichloromethane. The combined organic layers were dried over $\mathrm{Na}_{2} \mathrm{SO}_{4}$, filtered and concentrated to afford crude product, which was purified by preparative HPLC to afford the corresponding phosphorylated product.

\section{Reaction of 32 with 3-phenyl-1-propanol (Scheme 3a)}

A heat-gun dried and argon-flushed test tube equipped with a magnetic stirrer bar was charged with ethyl 3bromo-2-oxopropanoate ( $58.5 \mathrm{mg}, 300 \mu \mathrm{mol}, 3.0$ equiv.) and diethyl ether $(181 \mu \mathrm{L})$. Then, a solution of trimethyl phosphite $(37.2 \mathrm{mg}, 300 \mu \mathrm{mol}, 3.0$ equiv.) in diethyl ether $(181 \mu \mathrm{L})$ was added dropwise at a gently reflux rate, and the reaction mixture was stirred at r.t. for $1 \mathrm{~h}$. The solvent was removed under high vacuum, and the resulting 32 was used without further purification.

To the freshly-prepared 32, were added sequentially acetonitrile (1.0 mL, $0.10 \mathrm{M})$, 3-phenyl-1-propanol (13.2 $\mathrm{mg}, 100 \mu \mathrm{mol}, 1.0$ equiv.), and methyl nicotinate ( $13.7 \mathrm{mg}, 100 \mu \mathrm{mol}, 1.0$ equiv.). The mixture was warmed to $60{ }^{\circ} \mathrm{C}$ and stirred for $6 \mathrm{~h}$. Then the reaction mixture was cooled to r.t. and concentrated. After diluting adding 
saturated $\mathrm{Na}_{2} \mathrm{CO}_{3}$ aq, the mixture was extracted three times with diethyl ether (organic layer 1). The aqueous layer was acidified with concentrated $\mathrm{HCl}$ to $\mathrm{pH} 1$, and extracted five times with dichloromethane (organic layer 2). The two organic layers ( 1 and 2 ) were dried over $\mathrm{Na}_{2} \mathrm{SO}_{4}$, filtered, concentrated, and analyzed with ${ }^{1} \mathrm{H} \mathrm{NMR}$, respectively.

\section{Detection of 33 with LC-MS (Scheme 3b).}

To the freshly-prepared dimethyl phosphoenolpyruvate (3.0 equiv.), were added sequentially acetonitrile (1.0 $\mathrm{mL}, 0.10 \mathrm{M}), 3$-phenyl-1-propanol (13.2 mg, $100 \mu \mathrm{mol}, 1.0$ equiv.), and methyl nicotinate (13.7 mg, $100 \mu \mathrm{mol}$, 1.0 equiv.). The mixture was warmed to $60^{\circ} \mathrm{C}$ and stirred for $15 \mathrm{~min}$. Then $1.0 \mu \mathrm{L}$ of the reaction mixture was diluted with $99.0 \mu \mathrm{L}$ acetonitrile, and was analyzed by LC-MS (ESI-MS) using a gradient elution method of 2 $-90 \%$ acetonitrile over $9 \min$. 


\section{Characterization of phosphorylated products}<smiles>COP(=O)(O)OCCCc1ccccc1</smiles>

\section{Methyl 3-phenylpropyl hydrogen phosphate (7):}

General procedure $\mathbf{S}(9.64 \mathrm{mg}, 0.0418 \mathrm{mmol}, 42 \%$ isolated yield $)$

${ }^{1} \mathrm{H}$ NMR $\left(\mathrm{CDCl}_{3}, 400 \mathrm{MHz}\right) \delta$ 7.34-7.31 (m, 2H), 7.25-7.22 (m, 3H), 4.08 (dt, $J$ $=6.3 \mathrm{~Hz}, 6.4 \mathrm{~Hz}, 2 \mathrm{H}), 3.78(\mathrm{~d}, J=11.4 \mathrm{~Hz}, 3 \mathrm{H}), 2.76(\mathrm{t}, J=7.9 \mathrm{~Hz}, 2 \mathrm{H}), 2.07-2.00(\mathrm{~m}, 2 \mathrm{H}) ;{ }^{13} \mathrm{C} \mathrm{NMR}\left(\mathrm{CDCl}_{3}\right.$, $100 \mathrm{MHz}) \delta 141.0,128.5,128.4,126.0,66.8(\mathrm{~d}, J=5.7 \mathrm{~Hz}), 53.9(\mathrm{~d}, J=5.7 \mathrm{~Hz}), 31.8(\mathrm{~d}, J=7.6 \mathrm{~Hz}), 31.6 ;{ }^{31} \mathrm{P}$ NMR $\left(\mathrm{CDCl}_{3}, 159 \mathrm{MHz}\right) \delta 1.9$; ESI-MS $\mathrm{m} / \mathrm{z} 229.1[\mathrm{M}-\mathrm{H}]^{-}$; HRMS calcd for $\left[\mathrm{C}_{10} \mathrm{H}_{14} \mathrm{O}_{4} \mathrm{P}\right]^{-}$requires $\mathrm{m} / \mathrm{z}$ 229.0635; found 229.0635.

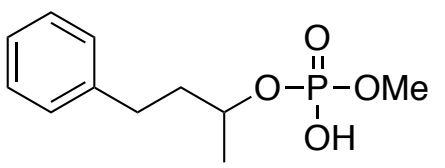

\section{Methyl (4-phenylbutan-2-yl) hydrogen phosphate (12):}

General procedure $\mathbf{S}(11.9 \mathrm{mg}, 0.0488 \mathrm{mmol}, 49 \%$ yield $)$

${ }^{1} \mathrm{H}$ NMR $\left(\mathrm{CDCl}_{3}, 400 \mathrm{MHz}\right) \delta$ 7.34-7.30 (m, 2H), 7.24-7.20 (m, 3H), 4.56-4.49 $(\mathrm{m}, 1 \mathrm{H}), 3.78(\mathrm{~d}, J=11.4 \mathrm{~Hz}, 3 \mathrm{H}), 2.84-2.77(\mathrm{~m}, 1 \mathrm{H}), 2.74-2.66(\mathrm{~m}, 1 \mathrm{H}), 2.06-1.97(\mathrm{~m}, 1 \mathrm{H}), 1.91-1.82(\mathrm{~m}, 1 \mathrm{H})$, $1.41(\mathrm{~d}, J=6.4 \mathrm{~Hz}, 3 \mathrm{H}) ;{ }^{13} \mathrm{C} \mathrm{NMR}\left(\mathrm{CDCl}_{3}, 100 \mathrm{MHz}\right) \delta 141.5,128.4,128.4,125.9,75.5(\mathrm{~d}, J=5.7 \mathrm{~Hz}), 53.8$ $(\mathrm{d}, J=5.7 \mathrm{~Hz}), 39.2(\mathrm{~d}, J=6.7 \mathrm{~Hz}), 31.4,21.5(\mathrm{~d}, J=2.9 \mathrm{~Hz}) ;{ }^{31} \mathrm{P} \mathrm{NMR}\left(\mathrm{CDCl}_{3}, 159 \mathrm{MHz}\right) \delta 1.3$; ESI-MS $m / z$ $243.1[\mathrm{M}-\mathrm{H}]^{-}$; HRMS calcd for $\left[\mathrm{C}_{11} \mathrm{H}_{16} \mathrm{O}_{4} \mathrm{P}\right]^{-}$requires $m / z$ 243.0792; found 243.0792 .

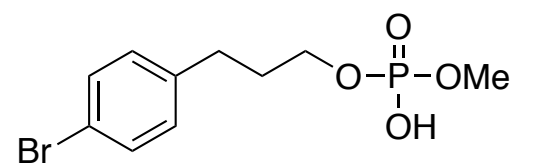

Methyl 3-(4-bromophenylpropyl) hydrogen phosphate (13):

General procedure $\mathbf{S}(13.0 \mathrm{mg}, 0.0421 \mathrm{mmol}, 42 \%$ yield $)$

${ }^{1} \mathrm{H}$ NMR $\left(\mathrm{CDCl}_{3}, 400 \mathrm{MHz}\right) \delta 7.37(\mathrm{~d}, J=8.5 \mathrm{~Hz}, 2 \mathrm{H}), 7.04(\mathrm{~d}, J=8.5 \mathrm{~Hz}$, 2H), $3.99(\mathrm{dt}, J=6.6 \mathrm{~Hz}, 6.6 \mathrm{~Hz}, 2 \mathrm{H}), 3.72(\mathrm{~d}, J=11.4 \mathrm{~Hz}, 3 \mathrm{H}), 2.65(\mathrm{t}, J=7.8 \mathrm{~Hz}, 2 \mathrm{H}), 1.97-1.90(\mathrm{~m}, 2 \mathrm{H}) ;{ }^{13} \mathrm{C}$ $\operatorname{NMR}\left(\mathrm{CDCl}_{3}, 100 \mathrm{MHz}\right) \delta 139.9,131.5,130.2,119.8,66.5(\mathrm{~d}, J=5.7 \mathrm{~Hz}), 54.0(\mathrm{~d}, J=5.7 \mathrm{~Hz}), 31.6(\mathrm{~d}, J=7.6$ $\mathrm{Hz}), 31.0 ;{ }^{31} \mathrm{P}$ NMR $\left(\mathrm{CDCl}_{3}, 159 \mathrm{MHz}\right) \delta 2.0$; ESI-MS $m / z 307.0[\mathrm{M}-\mathrm{H}]^{-}$; HRMS calcd for $\left[\mathrm{C}_{10} \mathrm{H}_{13} \mathrm{BrO}_{4} \mathrm{P}\right]^{-}$ requires $m / z 306.9740$; found 306.9740 .<smiles>COP(=O)(O)OCCCOc1ccccc1</smiles>

Methyl (3-phenoxypropyl) hydrogen phosphate (14):

General procedure $S$ (11.3 mg, $0.0459 \mathrm{mmol}, 46 \%$ yield)

${ }^{1} \mathrm{H} \mathrm{NMR}\left(\mathrm{CDCl}_{3}, 400 \mathrm{MHz}\right) \delta$ 7.26-7.23 (dd, $\left.J=7.3 \mathrm{~Hz}, 8.9 \mathrm{~Hz}, 2 \mathrm{H}\right), 6.91$ (t, $J=7.3 \mathrm{~Hz}, 1 \mathrm{H}), 6.86(\mathrm{~d}, J=8.9 \mathrm{~Hz}, 2 \mathrm{H}), 4.19(\mathrm{dt}, J=6.4 \mathrm{~Hz}, 6.4 \mathrm{~Hz}, 2 \mathrm{H}), 4.04(\mathrm{t}, J=6.0 \mathrm{~Hz}, 2 \mathrm{H}), 3.67$ (d, $J=11.4 \mathrm{~Hz}, 3 \mathrm{H}), 2.14-2.08(\mathrm{~m}, 2 \mathrm{H}) ;{ }^{13} \mathrm{C} \mathrm{NMR}\left(\mathrm{CDCl}_{3}, 100 \mathrm{MHz}\right) \delta 158.7,129.4,120.8,114.4,64.2(\mathrm{~d}, J=5.7$ $\mathrm{Hz}), 63.5,53.9$ (d, $J=5.7 \mathrm{~Hz}), 30.1(\mathrm{~d}, J=7.6 \mathrm{~Hz}) ;{ }^{31} \mathrm{P}$ NMR $\left(\mathrm{CD}_{3} \mathrm{OD}, 159 \mathrm{MHz}\right) \delta 0.2$; ESI-MS $m / z 245.1$ $[\mathrm{M}-\mathrm{H}]^{-}$; HRMS calcd for $\left[\mathrm{C}_{10} \mathrm{H}_{14} \mathrm{O}_{5} \mathrm{P}\right]^{-}$requires $\mathrm{m} / \mathrm{z} 245.0584$; found 245.0584 . 
<smiles>COP(=O)(O)OCCc1ccc(F)cc1</smiles>

$8.8 \mathrm{~Hz}, 8.8 \mathrm{~Hz}, 2 \mathrm{H}), 4.14(\mathrm{dt}, J=7.2 \mathrm{~Hz}, 7.2 \mathrm{~Hz}, 2 \mathrm{H}), 3.60(\mathrm{~d}, J=11.4 \mathrm{~Hz}, 3 \mathrm{H}), 2.92(\mathrm{t}, J=7.2 \mathrm{~Hz}, 2 \mathrm{H}) ;{ }^{13} \mathrm{C}$ $\operatorname{NMR}\left(\mathrm{CDCl}_{3}, 100 \mathrm{MHz}\right) \delta 161.7(\mathrm{~d}, J=248.9 \mathrm{~Hz}), 132.8(\mathrm{~d}, J=2.9 \mathrm{~Hz}), 130.4(\mathrm{~d}, J=7.6 \mathrm{~Hz}), 115.3(\mathrm{~d}, J=$ 21.9 Hz), $67.7(\mathrm{~d}, J=6.7 \mathrm{~Hz}), 53.9(\mathrm{~d}, J=6.7 \mathrm{~Hz}), 35.8(\mathrm{~d}, J=6.7 \mathrm{~Hz}) ;{ }^{31} \mathrm{P} \mathrm{NMR}\left(\mathrm{CDCl}_{3}, 159 \mathrm{MHz}\right) \delta 1.5$; ESIMS $m / z 233.1[\mathrm{M}-\mathrm{H}]^{-}$; HRMS calcd for $\left[\mathrm{C}_{9} \mathrm{H}_{11} \mathrm{FO}_{4} \mathrm{P}\right]^{-}$requires $m / z$ 233.0385; found 233.0385.<smiles>COP(=O)(O)OCCc1ccc(Cl)cc1</smiles>

Methyl (4-chlorophenethyl) hydrogen phosphate (16):

General procedure $\mathbf{S}(11.3 \mathrm{mg}, 0.0451 \mathrm{mmol}, 45 \%$ yield $)$

${ }^{1} \mathrm{H}$ NMR $\left(\mathrm{CDCl}_{3}, 400 \mathrm{MHz}\right) \delta 7.24(\mathrm{~d}, J=8.7 \mathrm{~Hz}, 2 \mathrm{H}), 7.13(\mathrm{~d}, J=8.7 \mathrm{~Hz}$, $2 \mathrm{H}), 4.14(\mathrm{dt}, J=6.9 \mathrm{~Hz}, 6.9 \mathrm{~Hz}, 2 \mathrm{H}), 3.61(\mathrm{~d}, J=11.4 \mathrm{~Hz}, 3 \mathrm{H}), 2.92(\mathrm{t}, J=6.9 \mathrm{~Hz}, 2 \mathrm{H}) ;{ }^{13} \mathrm{C} \mathrm{NMR}_{(\mathrm{CDCl}}, 100$ $\mathrm{MHz}) \delta 135.6,132.5,130.3,128.6,67.5(\mathrm{~d}, J=5.7 \mathrm{~Hz}), 53.9(\mathrm{~d}, J=5.7 \mathrm{~Hz}), 35.9(\mathrm{~d}, J=6.7 \mathrm{~Hz}) ;{ }^{31} \mathrm{P} \mathrm{NMR}$ $\left(\mathrm{CDCl}_{3}, 159 \mathrm{MHz}\right) \delta 1.4 ;$ ESI-MS $m / z 249.1[\mathrm{M}-\mathrm{H}]^{-} ; \mathrm{HRMS}$ calcd for $\left[\mathrm{C}_{9} \mathrm{H}_{11} \mathrm{ClO}_{4} \mathrm{P}\right]^{-}$requires $m / z 249.0089$; found 249.0089 .

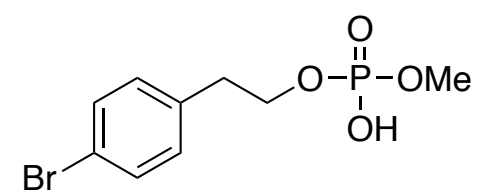

Methyl (4-bromophenethyl) hydrogen phosphate (17):

General procedure $\mathbf{S}(11.9 \mathrm{mg}, 0.0403 \mathrm{mmol}, 40 \%$ yield $)$

${ }^{1} \mathrm{H}$ NMR $\left(\mathrm{CDCl}_{3}, 400 \mathrm{MHz}\right) \delta 7.33(\mathrm{~d}, J=8.5 \mathrm{~Hz}, 2 \mathrm{H}), 7.02(\mathrm{~d}, J=8.5 \mathrm{~Hz}$, 2H), $4.07(\mathrm{dt}, J=6.9 \mathrm{~Hz}, 6.9 \mathrm{~Hz}, 2 \mathrm{H}), 3.54(\mathrm{~d}, J=11.0 \mathrm{~Hz}, 2 \mathrm{H}), 2.84(\mathrm{t}, J=6.9 \mathrm{~Hz}, 2 \mathrm{H}) ;{ }^{13} \mathrm{C} \mathrm{NMR}\left(\mathrm{CDCl}_{3}, 100\right.$ $\mathrm{MHz}) \delta 136.2,131.5,130.7,120.5,67.3(\mathrm{~d}, J=5.7 \mathrm{~Hz}), 53.9(\mathrm{~d}, J=5.7 \mathrm{~Hz}), 36.0(\mathrm{~d}, J=7.6 \mathrm{~Hz}) ;{ }^{31} \mathrm{P} \mathrm{NMR}$ $\left(\mathrm{CDCl}_{3}, 159 \mathrm{MHz}\right) \delta$ 1.2; ESI-MS $m / z 294.1[\mathrm{M}-\mathrm{H}]^{-}$; HRMS calcd for $\left[\mathrm{C}_{9} \mathrm{H}_{11} \mathrm{BrO}_{4} \mathrm{P}\right]^{-}$requires $m / z$ 292.9584; found 292.9584 .

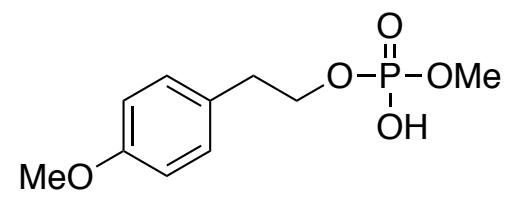

\section{Methyl (4-methoxyphenethyl) hydrogen phosphate (18):}

General procedure $\mathbf{S}(9.08 \mathrm{mg}, 0.0368 \mathrm{mmol}, 37 \%$ yield $)$

${ }^{1} \mathrm{H} \mathrm{NMR}\left(\mathrm{CDCl}_{3}, 400 \mathrm{MHz}\right) \delta 7.10(\mathrm{~d}, J=8.7 \mathrm{~Hz}, 2 \mathrm{H}), 6.80(\mathrm{~d}, J=8.7 \mathrm{~Hz}$, 2H), $4.12(\mathrm{dt}, J=7.1 \mathrm{~Hz}, 7.1 \mathrm{~Hz}, 2 \mathrm{H}), 3.75(\mathrm{~s}, 3 \mathrm{H}), 3.60(\mathrm{~d}, J=11.0,3 \mathrm{H}), 2.89(\mathrm{t}, J=7.1 \mathrm{~Hz}, 2 \mathrm{H}) ;{ }^{13} \mathrm{C} \mathrm{NMR}$ $\left(\mathrm{CDCl}_{3}, 100 \mathrm{MHz}\right) \delta 158.3,130.0,129.2,113.9,68.0(\mathrm{~d}, J=5.7 \mathrm{~Hz}), 55.2,53.8(\mathrm{~d}, J=4.8 \mathrm{~Hz}), 35.8(\mathrm{~d}, J=6.7$ $\mathrm{Hz}) ;{ }^{31} \mathrm{P} \mathrm{NMR}\left(\mathrm{CDCl}_{3}, 159 \mathrm{MHz}\right) \delta 1.6$; ESI-MS $m / z 245.1[\mathrm{M}-\mathrm{H}]^{-}$; HRMS calcd for $\left[\mathrm{C}_{10} \mathrm{H}_{14} \mathrm{O}_{5} \mathrm{P}\right]^{-}$requires $m / z$ 245.0584 ; found 245.0584 . 
<smiles>COP(=O)(O)OCCc1ccc([N+](=O)[O-])cc1</smiles>

Methyl (4-nitrophenethyl) hydrogen phosphate (19):

General procedure $\mathbf{S}$ ( $8.98 \mathrm{mg}, 0.0344 \mathrm{mmol}, 34 \%$ yield)

${ }^{1} \mathrm{H} \mathrm{NMR}\left(\mathrm{CDCl}_{3}, 400 \mathrm{MHz}\right) \delta 8.08(\mathrm{~d}, J=8.5 \mathrm{~Hz}, 2 \mathrm{H}), 7.32(\mathrm{~d}, J=8.5$

$\mathrm{Hz}, 2 \mathrm{H}), 4.16$ (dt, $J=6.9 \mathrm{~Hz}, 6.9 \mathrm{~Hz}, 2 \mathrm{H}), 3.55$, (d, $J=11.4 \mathrm{~Hz}, 3 \mathrm{H}), 3.00$

$(\mathrm{t}, J=6.9 \mathrm{~Hz}, 2 \mathrm{H}) ;{ }^{13} \mathrm{C} \mathrm{NMR}\left(\mathrm{CDCl}_{3}, 100 \mathrm{MHz}\right) \delta 146.9,145.0,129.9,123.7,66.8(\mathrm{~d}, J=5.7 \mathrm{~Hz}), 54.0(\mathrm{~d}, J=$ $5.7 \mathrm{~Hz}), 36.3(\mathrm{~d}, J=7.6 \mathrm{~Hz}) ;{ }^{31} \mathrm{P}$ NMR $\left(\mathrm{CDCl}_{3}, 159 \mathrm{MHz}\right) \delta 1.2$; ESI-MS $m / z 260.0[\mathrm{M}-\mathrm{H}]^{-}$; HRMS calcd for $\left[\mathrm{C}_{9} \mathrm{H}_{11} \mathrm{NO}_{6} \mathrm{P}\right]^{-}$requires $\mathrm{m} / z$ 260.0330; found 260.0330 .

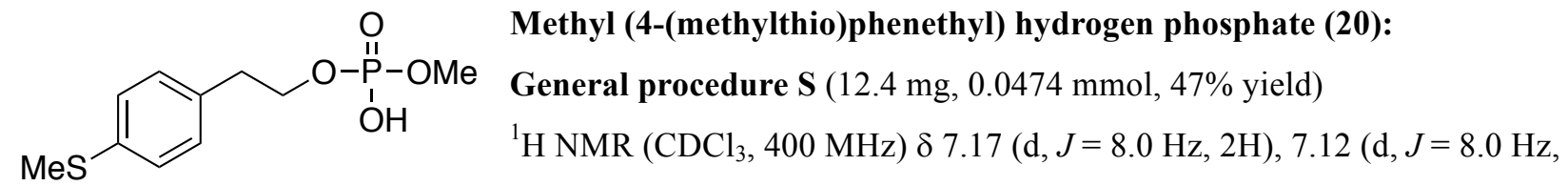
2H), 4.17 (dt, $J=7.3 \mathrm{~Hz}, 7.3 \mathrm{~Hz}, 2 \mathrm{H}), 3.63$ (d, $J=11.4 \mathrm{~Hz}, 3 \mathrm{H}), 2.93(\mathrm{t}, J=4.7 \mathrm{~Hz}, 2 \mathrm{H}), 2.44(\mathrm{~s}, 3 \mathrm{H}) ;{ }^{13} \mathrm{C} \mathrm{NMR}$ $\left(\mathrm{CDCl}_{3}, 100 \mathrm{MHz}\right) \delta 136.6,133.9,129.5,126.9,67.8(\mathrm{~d}, J=6.7 \mathrm{~Hz}), 54.0(\mathrm{~d}, J=5.7 \mathrm{~Hz}), 36.0(\mathrm{~d}, J=7.6 \mathrm{~Hz})$, $16.0 ;{ }^{31} \mathrm{P} \mathrm{NMR}\left(\mathrm{CDCl}_{3}, 159 \mathrm{MHz}\right) \delta 1.5$; ESI-MS $m / z 261.1[\mathrm{M}-\mathrm{H}]^{-}$; HRMS calcd for $\left[\mathrm{C}_{10} \mathrm{H}_{14} \mathrm{O}_{4} \mathrm{PS}\right]^{-}$requires $\mathrm{m} / \mathrm{z} 261.0356$; found 261.0356 .

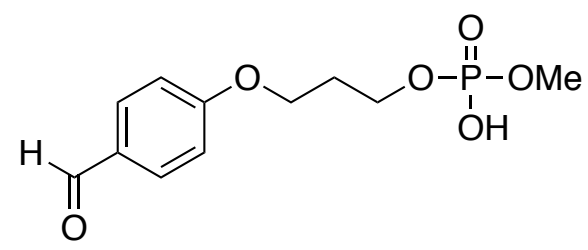

3-(4-Formylphenoxy)propyl methyl hydrogen phosphate (21):

General procedure $\mathbf{S}(13.2 \mathrm{mg}, 0.0482 \mathrm{mmol}, 48 \%$ yield)

${ }^{1} \mathrm{H} \mathrm{NMR}\left(\mathrm{CDCl}_{3}, 400 \mathrm{MHz}\right) \delta 9.85(\mathrm{~s}, 1 \mathrm{H}), 7.80(\mathrm{~d}, J=8.5 \mathrm{~Hz}, 2 \mathrm{H})$, $6.97(\mathrm{~d}, J=8.5 \mathrm{~Hz}, 2 \mathrm{H}), 4.24-4.19(\mathrm{~m}, 2 \mathrm{H}), 4.14(\mathrm{t}, J=5.7 \mathrm{~Hz}, 2 \mathrm{H})$, $3.69(\mathrm{~d}, J=11.0 \mathrm{~Hz}, 3 \mathrm{H}), 2.17-2.14(\mathrm{~m}, 2 \mathrm{H}) ;{ }^{13} \mathrm{C} \mathrm{NMR}\left(\mathrm{CDCl}_{3}, 100 \mathrm{MHz}\right) \delta 190.9,163.7,132.0,130.0,114.7$, $64.0(\mathrm{~d}, J=4.8 \mathrm{~Hz}), 64.0,54.1(\mathrm{~d}, J=3.8 \mathrm{~Hz}), 29.8(\mathrm{~d}, J=6.7 \mathrm{~Hz}) ;{ }^{31} \mathrm{P} \mathrm{NMR}\left(\mathrm{CDCl}_{3}, 159 \mathrm{MHz}\right) \delta 1.6$; ESI-MS $\mathrm{m} / z 273.1[\mathrm{M}-\mathrm{H}]^{-} ; \mathrm{HRMS}$ calcd for $\left[\mathrm{C}_{11} \mathrm{H}_{14} \mathrm{O}_{6} \mathrm{P}\right]^{-}$requires $\mathrm{m} / z$ 273.0534; found 273.0534.

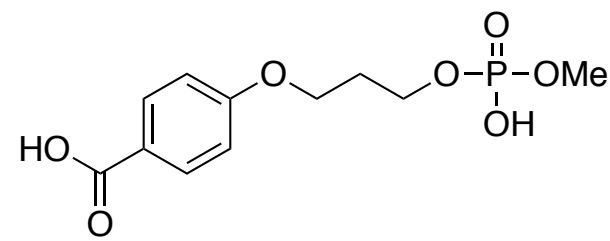

4-(3-((Hydroxy(methoxy)phosphoryl)oxy)propoxy)benzoic acid (22):

General procedure $\mathbf{S}(14.2 \mathrm{mg}, 0.0489 \mathrm{mmol}, 49 \%$ yield)

${ }^{1} \mathrm{H}$ NMR $\left(\mathrm{CD}_{3} \mathrm{OD}, 400 \mathrm{MHz}\right) \delta 7.87(\mathrm{~d}, J=8.9 \mathrm{~Hz}, 2 \mathrm{H}), 6.90(\mathrm{~d}, 8.9$ $\mathrm{Hz}, 2 \mathrm{H}), 4.11-4.07(\mathrm{~m}, 4 \mathrm{H}), 3.57(\mathrm{~d}, J=11.4 \mathrm{~Hz}, 3 \mathrm{H}), 2.09-2.03(\mathrm{~m}, 2 \mathrm{H}) ;{ }^{13} \mathrm{C} \mathrm{NMR}\left(\mathrm{CD}_{3} \mathrm{OD}, 100 \mathrm{MHz}\right) \delta 169.8$, 164.3, 132.9, 124.1, 115.2, 65.2, $64.7(\mathrm{~d}, J=5.7 \mathrm{~Hz}), 54.2(\mathrm{~d}, J=5.7 \mathrm{~Hz}), 31.2(\mathrm{~d}, J=6.7 \mathrm{~Hz}) ;{ }^{31} \mathrm{P} \mathrm{NMR}\left(\mathrm{CD}_{3} \mathrm{OD}\right.$, $159 \mathrm{MHz}) \delta 0.7$; ESI-MS $\mathrm{m} / \mathrm{z} 289.1[\mathrm{M}-\mathrm{H}]^{-}$; HRMS calcd for $\left[\mathrm{C}_{11} \mathrm{H}_{14} \mathrm{O}_{7} \mathrm{P}\right]^{-}$requires $\mathrm{m} / z$ 289.0483; found 289.0483 . 
<smiles>COC(=O)c1ccc(OCCCOP(=O)(O)OC)cc1</smiles>

Methyl 4-(3-((hydroxy(methoxy)phosphoryl)oxy)propoxy) benzoate (23):

General procedure $\mathbf{S}(15.7 \mathrm{mg}, 0.0516 \mathrm{mmol}, 52 \%$ yield $)$

${ }^{1} \mathrm{H} \mathrm{NMR}\left(\mathrm{CDCl}_{3}, 400 \mathrm{MHz}\right) \delta 7.95(\mathrm{~d}, J=8.7 \mathrm{~Hz}, 2 \mathrm{H}), 6.88(\mathrm{~d}, J$ $=8.7 \mathrm{~Hz}, 2 \mathrm{H}), 4.21(\mathrm{dt}, J=6.4 \mathrm{~Hz}, 6.4 \mathrm{~Hz}, 2 \mathrm{H}), 4.10(\mathrm{t}, J=6.4 \mathrm{~Hz}, 2 \mathrm{H}), 3.86(\mathrm{~s}, 3 \mathrm{H}), 3.68(\mathrm{~d}, J=11.4 \mathrm{~Hz}, 3 \mathrm{H})$, 2.17-2.11 (m, 2H); ${ }^{13} \mathrm{C} \mathrm{NMR}\left(\mathrm{CDCl}_{3}, 100 \mathrm{MHz}\right) \delta 166.9,162.4,131.6,122.7,114.0,64.1(\mathrm{~d}, J=4.8 \mathrm{~Hz}), 63.7$, $54.0(\mathrm{~d}, J=5.7 \mathrm{~Hz}), 51.9,29.9(\mathrm{~d}, J=6.7 \mathrm{~Hz}) ;{ }^{31} \mathrm{P} \mathrm{NMR}\left(\mathrm{CDCl}_{3}, 159 \mathrm{MHz}\right) \delta 1.6$; ESI-MS m/z $303.1[\mathrm{M}-\mathrm{H}]^{-}$; HRMS calcd for $\left[\mathrm{C}_{12} \mathrm{H}_{16} \mathrm{O}_{7} \mathrm{P}\right]^{-}$requires $m / z$ 303.0639; found 303.0639 .

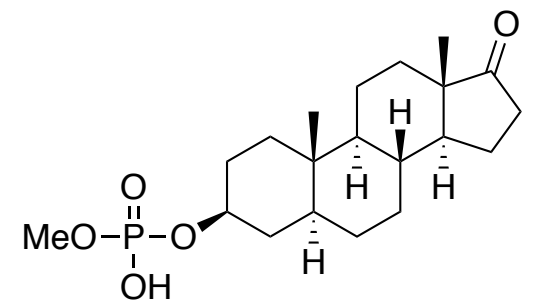

$(3 S, 5 S, 8 R, 9 S, 10 S, 13 S, 14 S)$-10,13-Dimethyl-17-oxohexadecahydro-1 $H$ cyclopenta[a]phenanthren-3-yl methyl hydrogen phosphate (24):

General procedure $\mathbf{S}$ (19.0 mg, $0.0494 \mathrm{mmol}, 49 \%$ yield)

${ }^{1} \mathrm{H} \mathrm{NMR}\left(\mathrm{CDCl}_{3}, 400 \mathrm{MHz}\right) \delta 4.24-4.21(\mathrm{~m}, 1 \mathrm{H}), 3.70$ (d, $\left.J=11.0 \mathrm{~Hz}, 3 \mathrm{H}\right)$, $2.41(\mathrm{dd}, J=9.2 \mathrm{~Hz}, 19.6 \mathrm{~Hz}, 1 \mathrm{H}), 2.08-1.85(\mathrm{~m}, 3 \mathrm{H}), 1.78-1.71(\mathrm{~m}, 4 \mathrm{H})$, $1.62-1.58(\mathrm{~m}, 2 \mathrm{H}), 1.53-1.41(\mathrm{~m}, 3 \mathrm{H}), 1.33-1.08(\mathrm{~m}, 6 \mathrm{H}), 1.00-0.89(\mathrm{~m}, 2 \mathrm{H}), 0.83(\mathrm{~s}, 3 \mathrm{H}), 0.81(\mathrm{~s}, 3 \mathrm{H}), 0.68-$ $0.62(\mathrm{~m}, 1 \mathrm{H}) ;{ }^{13} \mathrm{C} \mathrm{NMR}\left(\mathrm{CDCl}_{3}, 100 \mathrm{MHz}\right) \delta 221.2,78.1(\mathrm{~d}, J=5.7 \mathrm{~Hz}), 54 . .3,53.9(\mathrm{~d}, J=5.7 \mathrm{~Hz}), 51.3,47.7$, 44.6, 36.7, 35.8, 35.6 (d, $J=3.8 \mathrm{~Hz}), 35.4,34.9,31.5,30.8,29.1$ (d, $J=4.8 \mathrm{~Hz}), 28.2,21.7,20.4,13.8,12.2 ;{ }^{31} \mathrm{P}$ NMR $\left(\mathrm{CD}_{3} \mathrm{OD}, 159 \mathrm{MHz}\right) \delta 1.2$; ESI-MS $\mathrm{m} / z 383.3[\mathrm{M}-\mathrm{H}]^{-}$; HRMS calcd for $\left[\mathrm{C}_{20} \mathrm{H}_{32} \mathrm{O}_{5} \mathrm{P}\right]^{-}$requires $\mathrm{m} / z$ 383.1993; found 383.1993.

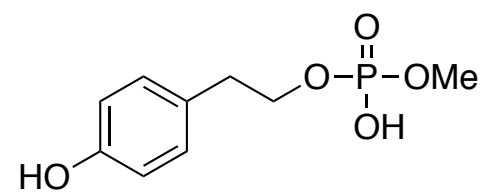

4-Hydroxyphenethyl methyl hydrogen phosphate (25):

General procedure $\mathbf{S}(7.49 \mathrm{mg}, 0.0323 \mathrm{mmol}, 32 \%$ yield $)$

${ }^{1} \mathrm{H}$ NMR $\left(\mathrm{CD}_{3} \mathrm{OD}, 400 \mathrm{MHz}\right) \delta 6.97(\mathrm{~d}, J=8.7 \mathrm{~Hz}, 2 \mathrm{H}), 6.62(\mathrm{~d}, J=8.7 \mathrm{~Hz}$, 2H), $4.01(\mathrm{dt}, J=7.1 \mathrm{~Hz}, 7.1 \mathrm{~Hz}, 2 \mathrm{H}), 3.49$ (d, $J=11.0 \mathrm{~Hz}, 3 \mathrm{H}), 2.77(\mathrm{t}, J=7.1 \mathrm{~Hz}, 2 \mathrm{H}) ;{ }^{13} \mathrm{C} \mathrm{NMR}\left(\mathrm{CD}_{3} \mathrm{OD}\right.$, $100 \mathrm{MHz}) \delta 157.2,131.1,129.6116 .2,69.2$ (d, $J=5.7 \mathrm{~Hz}), 54.2$ (d, $J=5.7 \mathrm{~Hz}), 36.9$ (d, $J=6.7 \mathrm{~Hz}) ;{ }^{31} \mathrm{P} \mathrm{NMR}$ $\left(\mathrm{CD}_{3} \mathrm{OD}, 159 \mathrm{MHz}\right) \delta$ 0.4; ESI-MS $\mathrm{m} / z 231.1[\mathrm{M}-\mathrm{H}]^{-}$; HRMS calcd for $\left[\mathrm{C}_{9} \mathrm{H}_{12} \mathrm{O}_{5} \mathrm{P}\right]^{-}$requires $\mathrm{m} / z$ 231.0428; found 231.0428 .

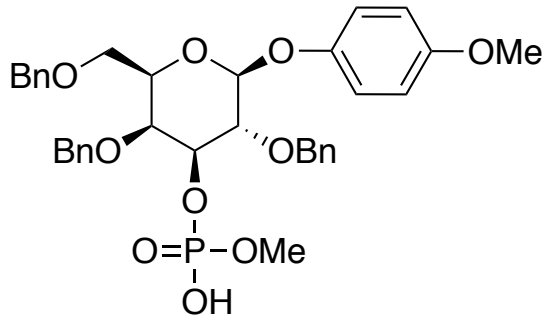

$(2 R, 3 S, 4 S, 5 R, 6 S)-3,5-B i s(b e n z y l o x y)-2-((b e n z y l o x y) m e t h y l)-6-(4-$ methoxyphenoxy)tetrahydro-2H-pyran-4-yl methyl hydrogen phosphate (26):

General procedure A (19.8 $\mathrm{mg}, 0.0304 \mathrm{mmol}, 30 \%$ yield $)$ ${ }^{1} \mathrm{H} \mathrm{NMR}\left(\mathrm{CDCl}_{3}, 400 \mathrm{MHz}\right) \delta$ 7.35-7.18 (m, 15H), $6.92(\mathrm{~d}, J=7.8,2 \mathrm{H})$, $6.71(\mathrm{~d}, J=7.8,2 \mathrm{H}), 4.92(\mathrm{~d}, J=11.4 \mathrm{~Hz}, 1 \mathrm{H}), 4.88(\mathrm{~d}, J=11.9 \mathrm{~Hz}, 1 \mathrm{H})$, 4.78-4.74 (m, 2H), 4.53 (d, $J=11.9 \mathrm{~Hz}), 4.28-4.29(\mathrm{~m}, 3 \mathrm{H}), 4.03(\mathrm{~d}, J=2.7 \mathrm{~Hz}, 1 \mathrm{H}), 3.98$ (dd, $J=8.7 \mathrm{~Hz}, 8.7$ 
$\mathrm{Hz}, \quad 1 \mathrm{H}), \quad 3.70(\mathrm{~s}, \quad 3 \mathrm{H}), \quad 3.56-3.49(\mathrm{~m}, 5 \mathrm{H}), \quad 3.43-3.41(\mathrm{~m}, 1 \mathrm{H}) ;{ }^{13} \mathrm{C} \quad \mathrm{NMR}\left(\mathrm{CDCl}_{3}, 100 \mathrm{MHz}\right)$ $\delta 155.2,151.3,138.1,137.9,137.8,128.4,128.3,128.3,128.2,127.8,127.7,127.7,118.4,114.4,102.6,79.7$ ( $\mathrm{d}, J=5.7 \mathrm{~Hz}), 77.4(\mathrm{~d}, J=6.7 \mathrm{~Hz}), 77.2,75.1$ (d, $J=7.6 \mathrm{~Hz}), 74.7,73.5,73.2,68.6,55.6,54.3(\mathrm{~d}, J=6.7 \mathrm{~Hz})$; ${ }^{31} \mathrm{P}$ NMR $\left(\mathrm{CDCl}_{3}, 159 \mathrm{MHz}\right) \delta 1.2$; ESI-MS $\mathrm{m} / z 325.2[\mathrm{M}-2 \mathrm{H}]^{2-}$; HRMS calcd for $\left[\mathrm{C}_{35} \mathrm{H}_{38} \mathrm{O}_{10} \mathrm{P}\right]^{-}$requires $m / z$ 649.2208; found 649.2208.

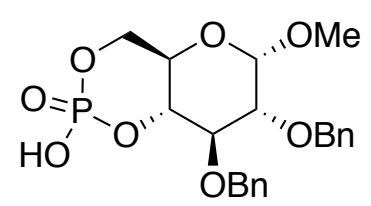

$(4 \mathrm{a} R, 6 S, 7 R, 8 R, 8 \mathrm{a} R)-7,8-\mathrm{Bis}(\mathrm{benzyloxy})-2-h y d r o x y-6-m e t h o x y h e x a h y d r o p y r a n o$ $[3,2-d][1,3,2]$ dioxaphosphinine 2-oxide (27):

General procedure A $(16.1 \mathrm{mg}, 0.0368 \mathrm{mmol}, 37 \%$ yield $)$

${ }^{1} \mathrm{H}$ NMR $\left(\mathrm{CDCl}_{3}, 400 \mathrm{MHz}\right) \delta$ 7.33-7.19 (m, 10H), $4.82(\mathrm{~d}, J=11.4 \mathrm{~Hz}, 1 \mathrm{H}), 4.76(\mathrm{~d}$, $J=12.4 \mathrm{~Hz}, 1 \mathrm{H}), 4.70(\mathrm{~d}, J=11.4 \mathrm{~Hz}, 1 \mathrm{H}), 4.56(\mathrm{~d}, J=12.4 \mathrm{~Hz}, 1 \mathrm{H}), 4.46(\mathrm{~d}, J=3.2 \mathrm{~Hz}, 1 \mathrm{H}), 4.23-4.10$ (m, $3 \mathrm{H}), 3.97-3.90(\mathrm{~m}, 2 \mathrm{H}), 3.41-3.39(\mathrm{~m}, 1 \mathrm{H}), 3.34(\mathrm{~s}, 3 \mathrm{H}) ;{ }^{13} \mathrm{C} \mathrm{NMR}\left(\mathrm{CDCl}_{3}, 100 \mathrm{MHz}\right) \delta 138.2,137.8,128.5$, 128.3, 128.1, 128.1, 128.0, 127.7, 98.9, $80.5(\mathrm{~d}, J=5.7 \mathrm{~Hz}), 78.6$ (d, $J=8.6 \mathrm{~Hz}), 78.4,75.5,74.0,68.4(J=6.8$ $\mathrm{Hz}), 62.3(\mathrm{~d}, J=5.7 \mathrm{~Hz}), 55.7 ;{ }^{31} \mathrm{P}$ NMR $\left(\mathrm{CDCl}_{3}, 159 \mathrm{MHz}\right) \delta-3.8$; ESI-MS $m / z 435.1[\mathrm{M}-\mathrm{H}]^{-}$; HRMS calcd for $\left[\mathrm{C}_{21} \mathrm{H}_{24} \mathrm{O}_{8} \mathrm{P}\right]^{-}$requires $m / z$ 435.1214; found 435.1214.<smiles>COC(=O)[C@H](COP(=O)(O)OC)NC(=O)OCc1ccccc1</smiles>

Methyl $N$-((benzyloxy)carbonyl)-O-(hydroxy(methoxy)phosphoryl)- $L$-serinate (28):

General procedure B (15.4 mg, $0.0445 \mathrm{mmol}, 45 \%$ yield)

${ }^{1} \mathrm{H}$ NMR $\left(\mathrm{CD}_{3} \mathrm{OD}, 400 \mathrm{MHz}\right) \delta$ 7.37-7.28 (m, 5H), $5.12(\mathrm{~s}, 2 \mathrm{H}), 4.54-4.52(\mathrm{~m}, 1 \mathrm{H}), 4.30-$ $4.24(\mathrm{~m}, 2 \mathrm{H}), 3.75(\mathrm{~s}, 3 \mathrm{H}), 3.65(\mathrm{~d}, J=11.4 \mathrm{~Hz}, 3 \mathrm{H}) ;{ }^{13} \mathrm{C} \mathrm{NMR}\left(\mathrm{CD}_{3} \mathrm{OD}, 100 \mathrm{MHz}\right)$ $\delta 171.2,158.4,138.1,129.5,129.1,128.9,67.8,67.2(\mathrm{~d}, J=5.7 \mathrm{~Hz}), 55.9(\mathrm{~d}, J=8.6 \mathrm{~Hz})$, $54.4(\mathrm{~d}, J=5.7 \mathrm{~Hz}), 53.1 ;{ }^{31} \mathrm{P}$ NMR $\left(\mathrm{CD}_{3} \mathrm{OD}, 159 \mathrm{MHz}\right) \delta 0.2$; ESI-MS $m / z 346.1[\mathrm{M}-\mathrm{H}]^{-}$; HRMS calcd for $\left[\mathrm{C}_{13} \mathrm{H}_{17} \mathrm{NO}_{8} \mathrm{P}\right]^{-}$requires $\mathrm{m} / \mathrm{z} 346.0697$; found 346.0697 .<smiles>COC(=O)[C@H](NC(=O)OCc1ccccc1)[C@H](C)OP(=O)(O)OC</smiles>

Methyl $N$-((benzyloxy)carbonyl)-O-(hydroxy(methoxy)phosphoryl)- $L$-threoninate (29):

General procedure B (15.0 $\mathrm{mg}, 0.0414 \mathrm{mmol}, 41 \%$ yield $)$

${ }^{1} \mathrm{H} \mathrm{NMR}\left(\mathrm{CDCl}_{3}, 400 \mathrm{MHz}\right) \delta$ 7.34-7.29 (m, 5H), $5.69(\mathrm{~d}, J=9.2 \mathrm{~Hz}, 1 \mathrm{H}), 5.14-5.08(\mathrm{~m}$, 2H), 4.96-4.93 (m, 1H), 4.47-4.44 (m, 1H), $3.74(\mathrm{~s}, 3 \mathrm{H}), 3.67$ (d, $J=11.4 \mathrm{~Hz}, 3 \mathrm{H}), 1.38$ $(\mathrm{d}, J=6.0 \mathrm{~Hz}, 3 \mathrm{H}) ;{ }^{13} \mathrm{C} \mathrm{NMR}\left(\mathrm{CDCl}_{3}, 100 \mathrm{MHz}\right) \delta 170.0,156.6,136.0,128.5,128.2,128.0,75.3(\mathrm{~d}, J=4.8$ $\mathrm{Hz}), 67.3,58.3(\mathrm{~d}, J=6.7 \mathrm{~Hz}), 54.3(\mathrm{~d}, J=6.5 \mathrm{~Hz}), 52.8,18.4(\mathrm{~d}, J=1.9 \mathrm{~Hz}) ;{ }^{31} \mathrm{P} \mathrm{NMR}\left(\mathrm{CDCl}_{3}, 159 \mathrm{MHz}\right)$ $\delta$ 0.2; ESI-MS $m / z 360.1[\mathrm{M}-\mathrm{H}]^{-}$; HRMS calcd for $\left[\mathrm{C}_{14} \mathrm{H}_{19} \mathrm{NO}_{8} \mathrm{P}\right]^{-}$requires $m / z$ 360.0854; found 360.0854 . 


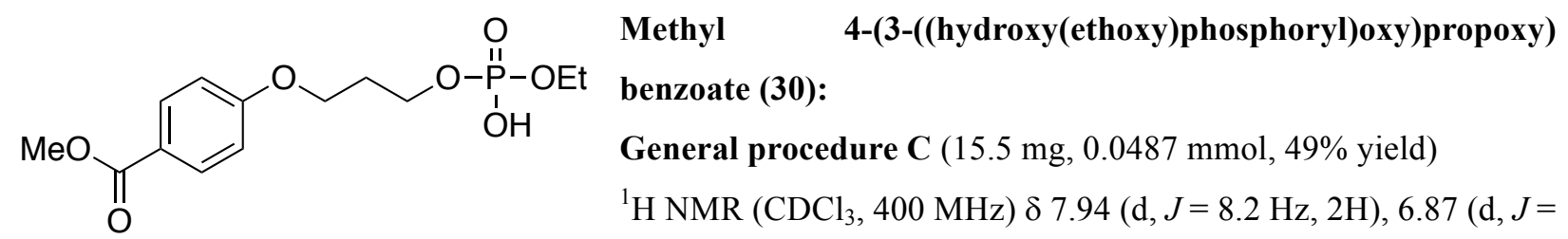
$8.2 \mathrm{~Hz}, 2 \mathrm{H}), 4.18(\mathrm{dt}, J=6.4 \mathrm{~Hz}, 6.4 \mathrm{~Hz}, 2 \mathrm{H}), 4.08(\mathrm{t}, J=6.0 \mathrm{~Hz}, 2 \mathrm{H}), 4.04-4.00$ (m, 2H), 3.85 (s, 3H), 2.15$2.09(\mathrm{~m}, 2 \mathrm{H}), 1.26(\mathrm{t}, J=7.3 \mathrm{~Hz}, 3 \mathrm{H}) ;{ }^{13} \mathrm{C} \mathrm{NMR}\left(\mathrm{CDCl}_{3}, 100 \mathrm{MHz}\right) \delta 166.8,162.4,131.5,122.6,114.0,63.9$ (d $=5.7 \mathrm{~Hz}), 63.8,63.7(\mathrm{~d}, J=5.7 \mathrm{~Hz}), 51.9,29.9(\mathrm{~d}, J=7.6 \mathrm{~Hz}), 16.0(\mathrm{~d}, J=6.7 \mathrm{~Hz}) ;{ }^{31} \mathrm{P} \mathrm{NMR}\left(\mathrm{CDCl}_{3}, 159 \mathrm{MHz}\right)$ $\delta$ 0.5; ESI-MS m/z $317.2[\mathrm{M}-\mathrm{H}]^{-}$; HRMS calcd for $\left[\mathrm{C}_{13} \mathrm{H}_{18} \mathrm{O}_{7} \mathrm{P}\right]^{-}$requires $m / z$ 317.0796; found 317.0796.

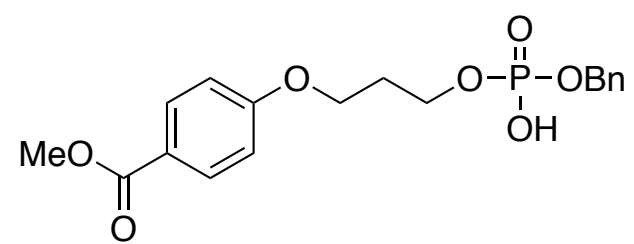

\section{Methyl 4-(3-((hydroxy(benzyloxy)phosphoryl)oxy)propoxy)} benzoate (31):

General procedure D $(16.2 \mathrm{mg}, 0.0426 \mathrm{mmol}, 43 \%$ yield $)$

${ }^{1} \mathrm{H} \mathrm{NMR}\left(\mathrm{CDCl}_{3}, 400 \mathrm{MHz}\right) \delta 7.88(\mathrm{~d}, J=7.3 \mathrm{~Hz}, 2 \mathrm{H}), 7.24-7.17$ (m, 5H), $6.77(\mathrm{~d}, J=7.3 \mathrm{~Hz}, 2 \mathrm{H}), 4.94(\mathrm{~d}, J=9.6 \mathrm{~Hz}, 2 \mathrm{H}), 4.10(\mathrm{dt}, J=6.3 \mathrm{~Hz}, 6.3 \mathrm{~Hz}, 2 \mathrm{H}), 3.95(\mathrm{t}, J=6.2 \mathrm{~Hz}$, 2H), 3.82 (s, 3H), 2.03-1.97 (m, 2H); ${ }^{13} \mathrm{C} \mathrm{NMR}\left(\mathrm{CDCl}_{3}, 100 \mathrm{MHz}\right) \delta 166.8,162.4,135.7$ (d, $\left.J=7.6 \mathrm{~Hz}\right), 131.5$, 128.5, 128.4, 127.7, 122.6, 114.0, 69.0 (d, $J=5.7 \mathrm{~Hz}), 64.1$ (d, $J=5.7 \mathrm{~Hz}), 63.8,51.8,29.8(\mathrm{~d}, J=7.6 \mathrm{~Hz}) ;{ }^{31} \mathrm{P}$ NMR $\left(\mathrm{CDCl}_{3}, 159 \mathrm{MHz}\right) \delta 0.6$; ESI-MS $\mathrm{m} / \mathrm{z} 379.1[\mathrm{M}-\mathrm{H}]^{-}$; HRMS calcd for $\left[\mathrm{C}_{18} \mathrm{H}_{20} \mathrm{O}_{7} \mathrm{P}\right]^{-}$requires $\mathrm{m} / \mathrm{z}$ 379.0952; found 379.0952. 

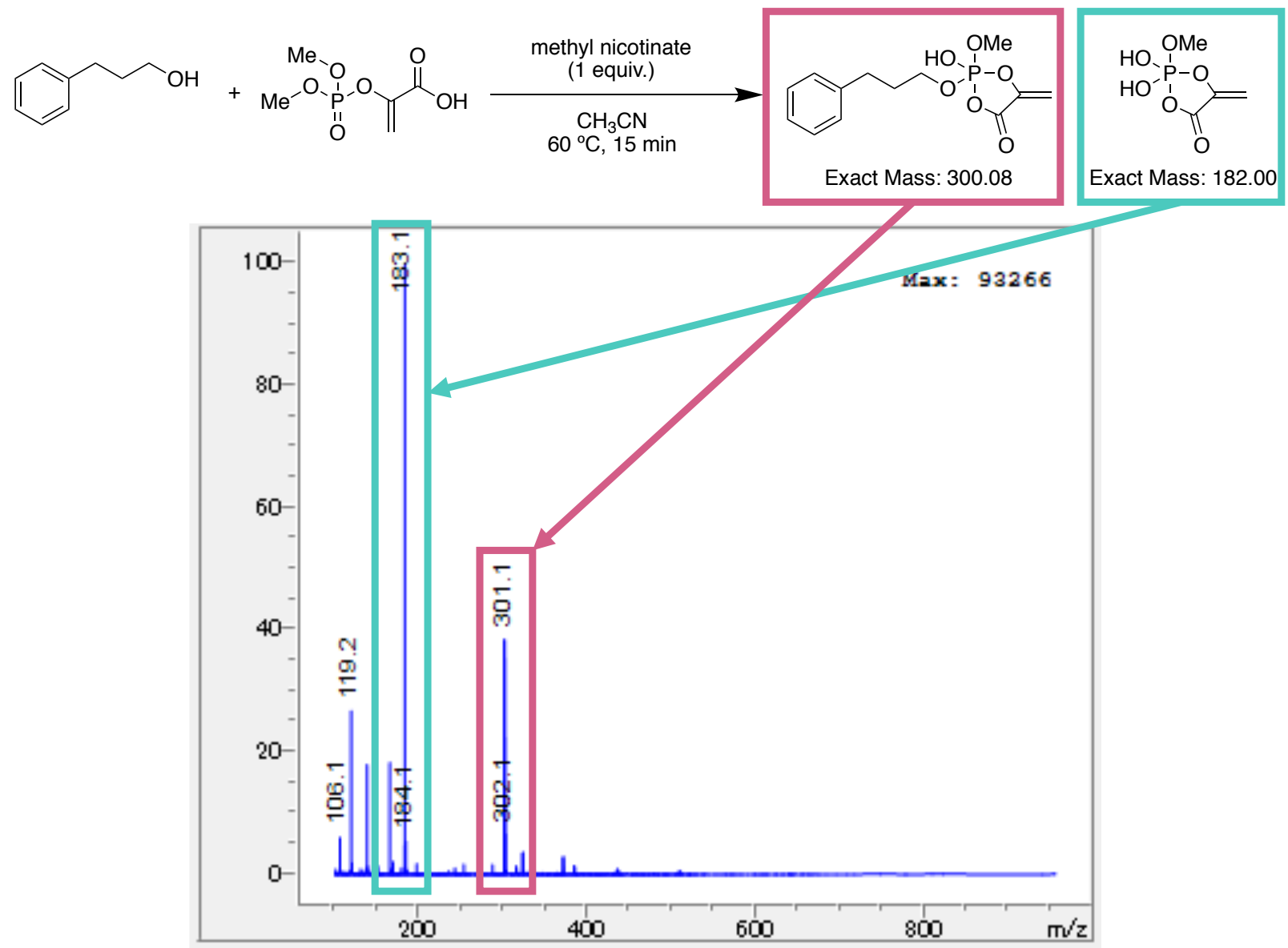

Figure S1. A MS spectrum after the reaction between 3-phenyl-1-propanol and dimethyl phosphoenolpyruvate at $60^{\circ} \mathbf{C}$ for 15 min. The MS spectrum showed peaks of $\mathrm{m} / \mathrm{z} 301.1$ and 183.1 , which correspond to pentacoordinate phosphorus intermediates shown in the scheme. 


\section{References}

1. Bernard L. Hirschbein; Francois P. Mazenod; and George M. Whitesides; Synthesis of Phosphoenolpyruvate and Its Use in Adenosine Triphosphate Cofactor Regeneration, $J$. Org. Chem. 1982, 47, 3765-3766. 

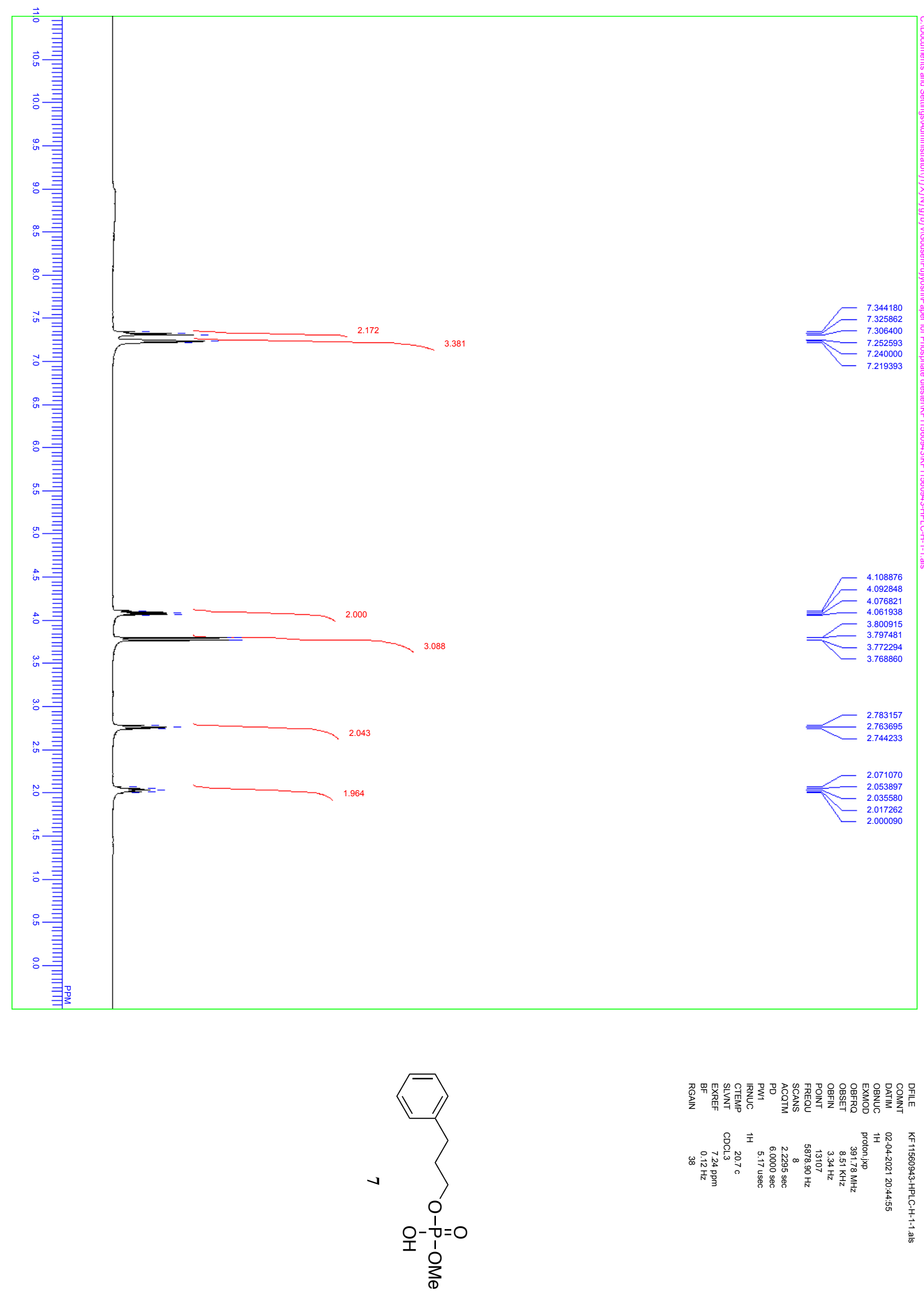

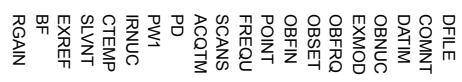

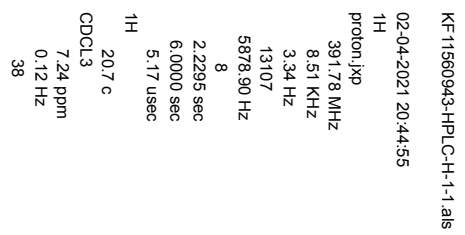



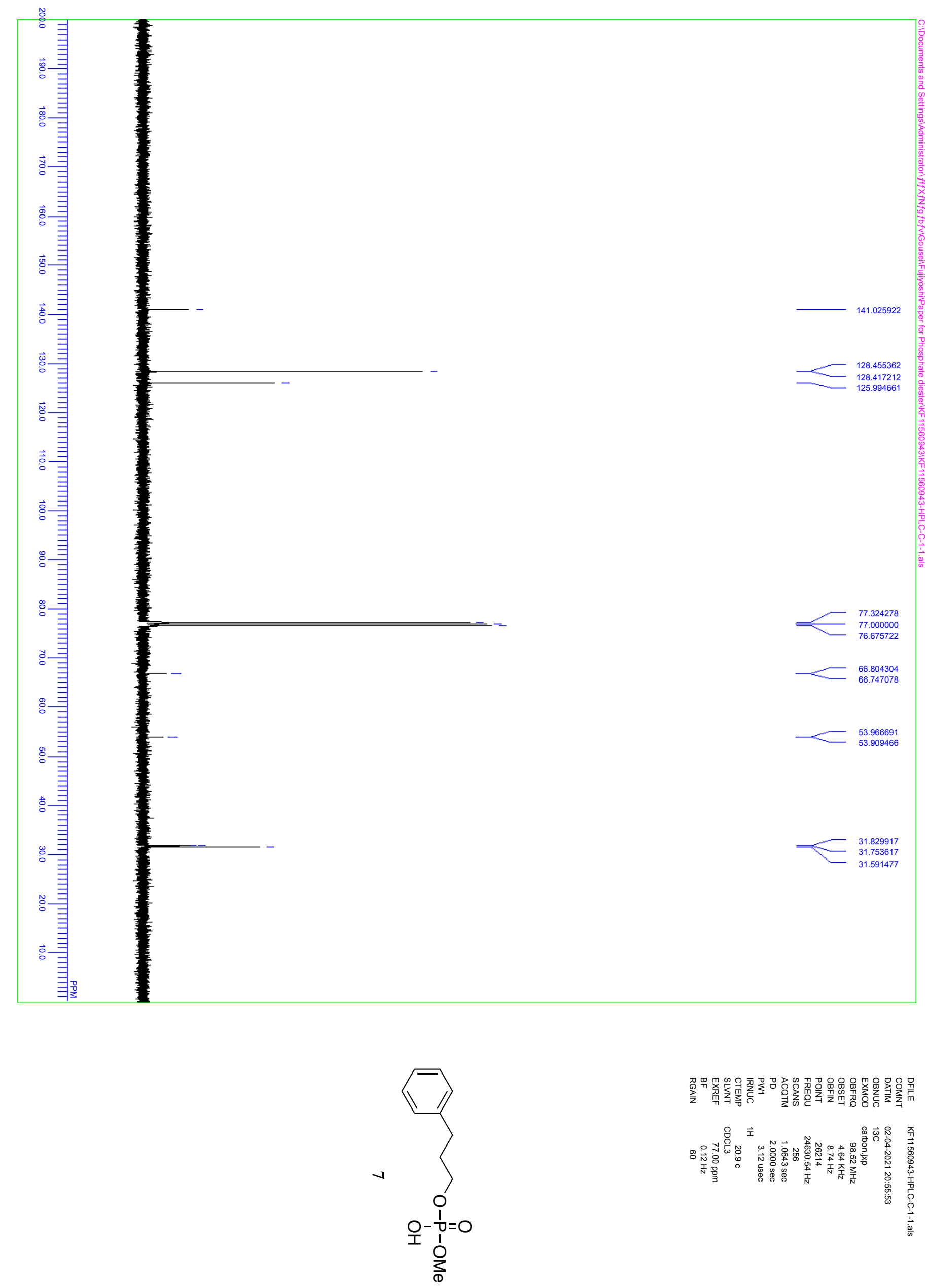

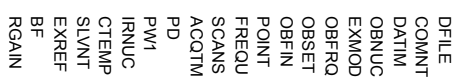

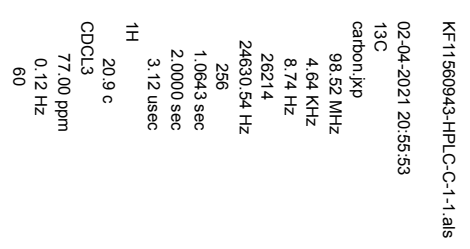



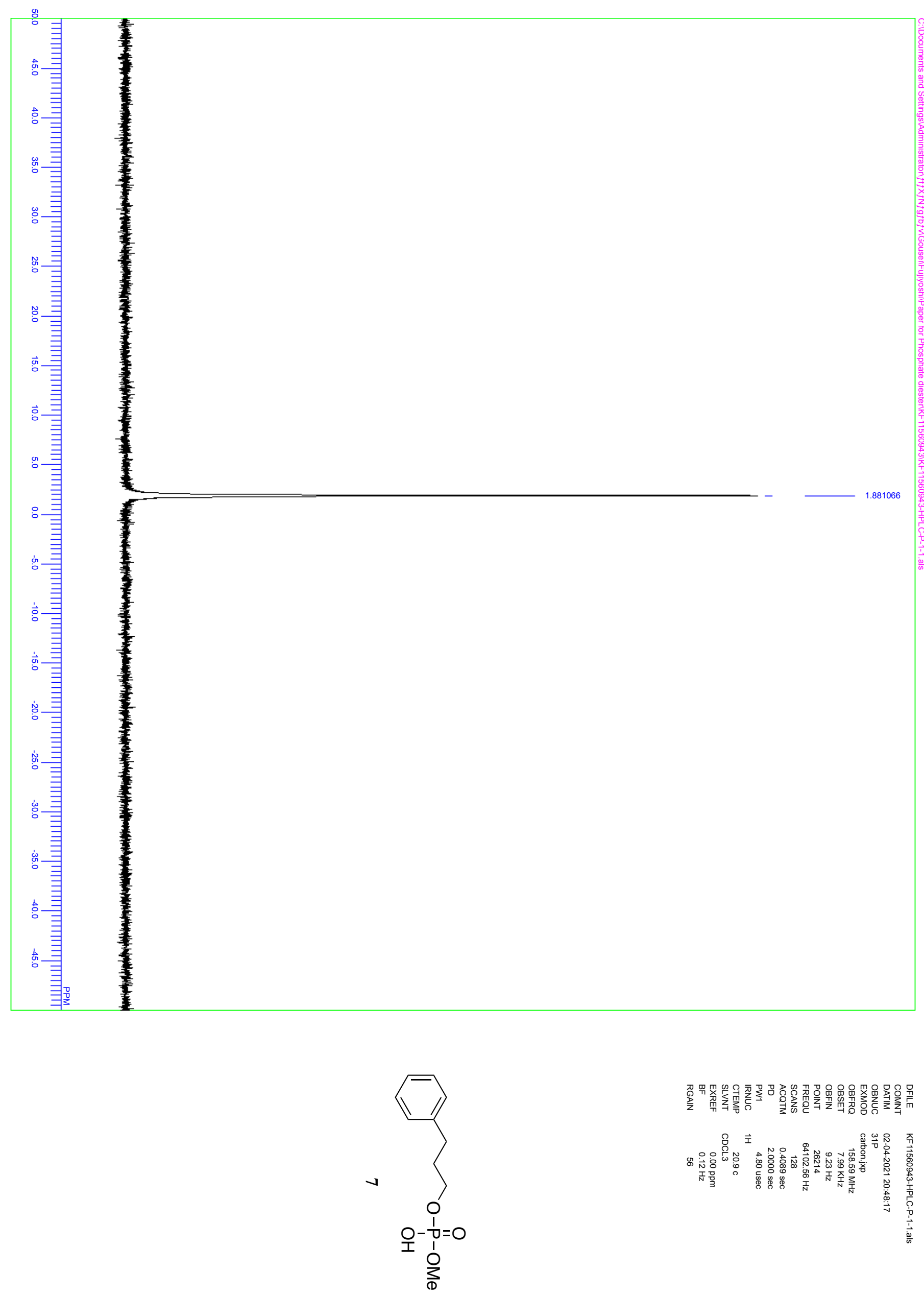

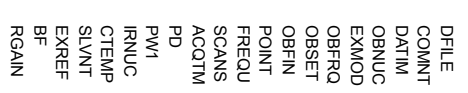

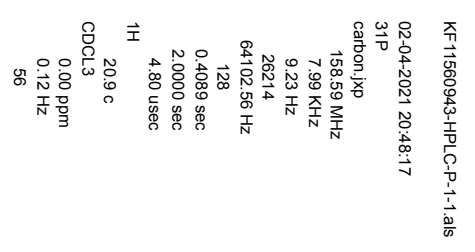



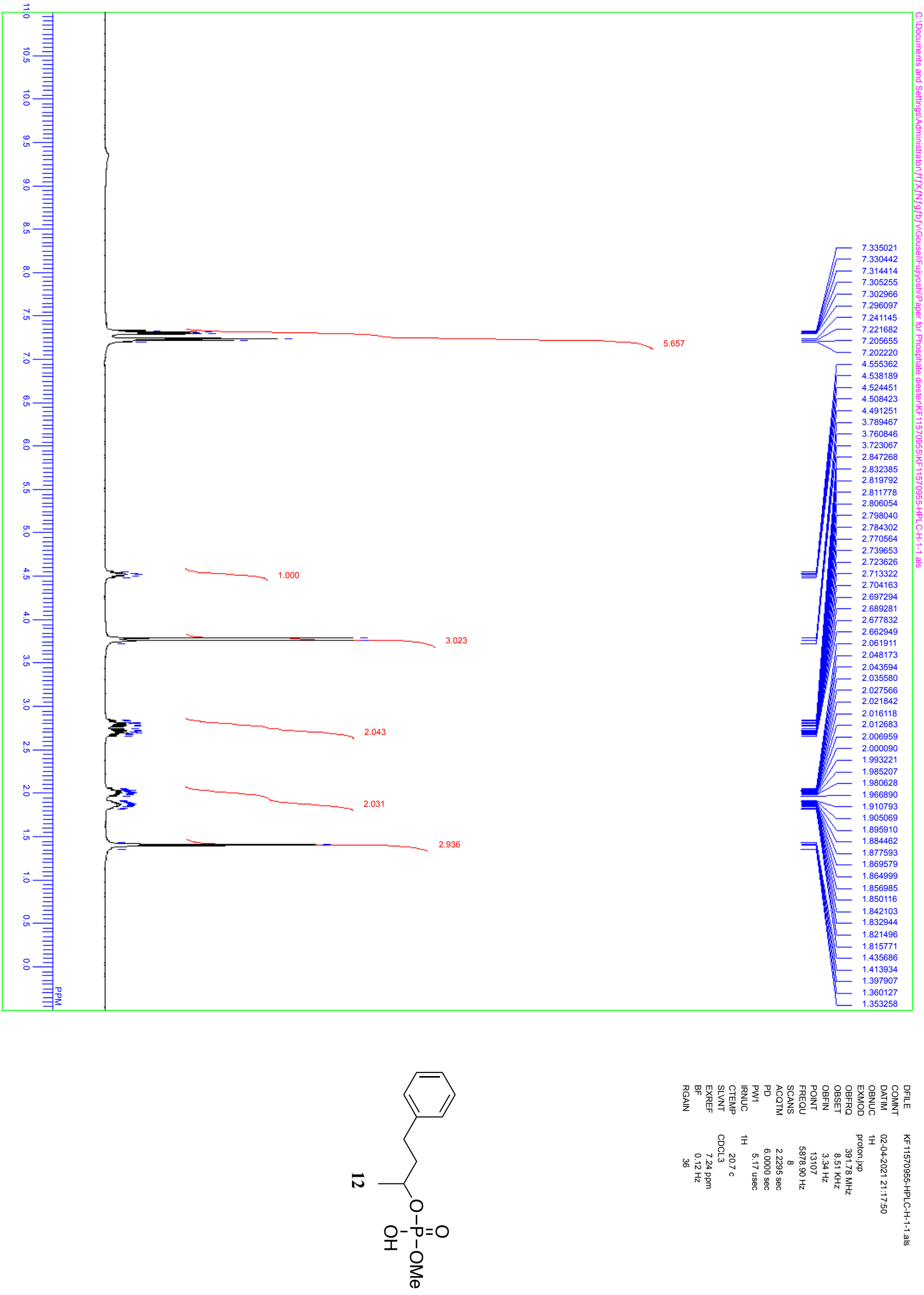

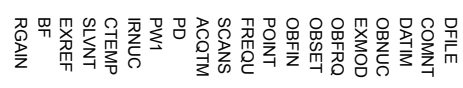

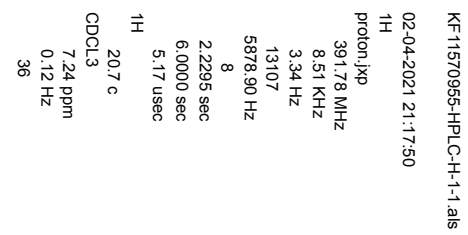



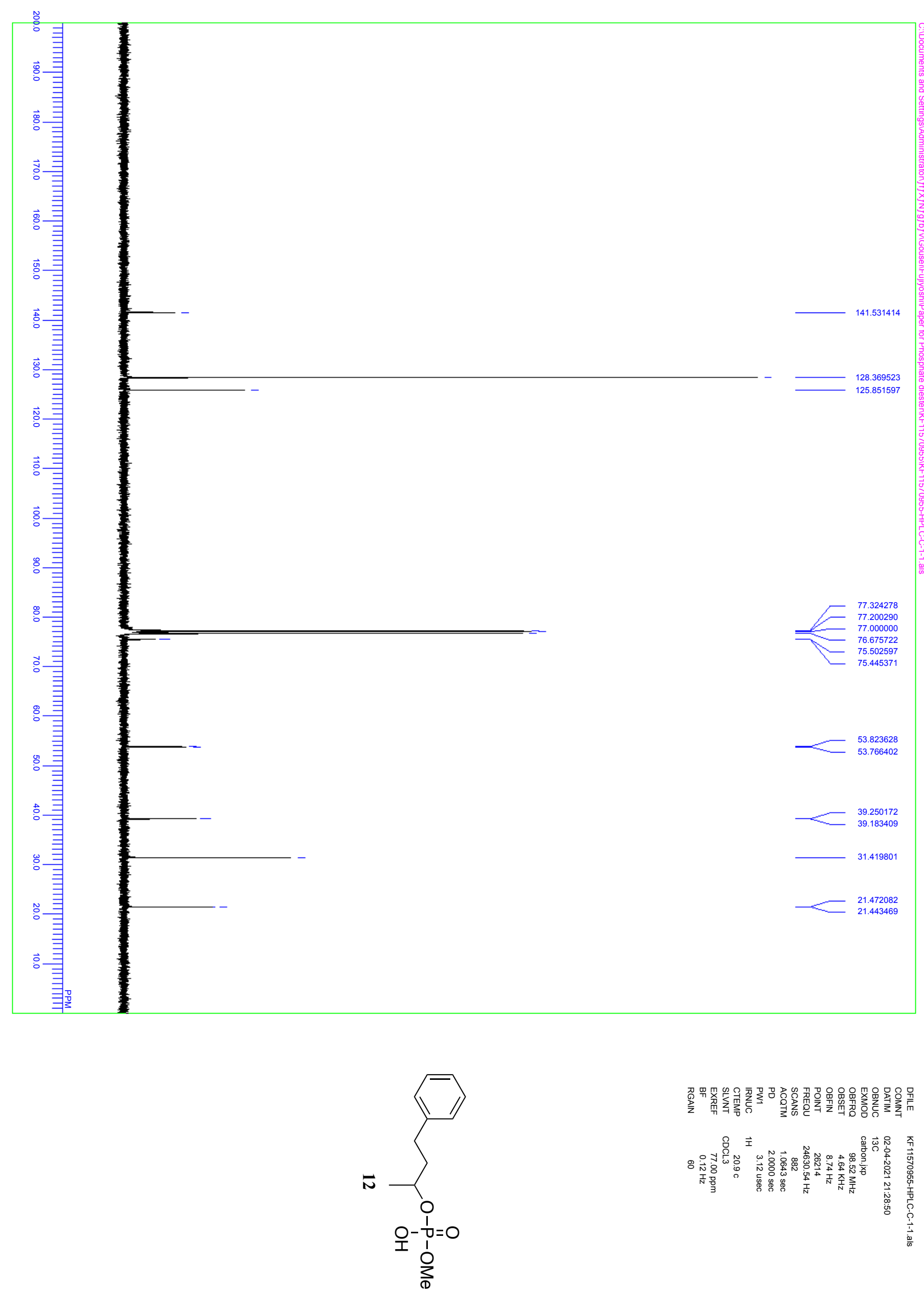

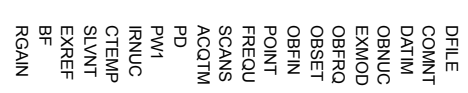

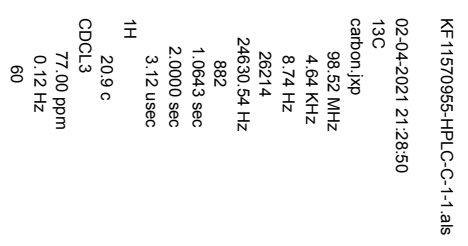



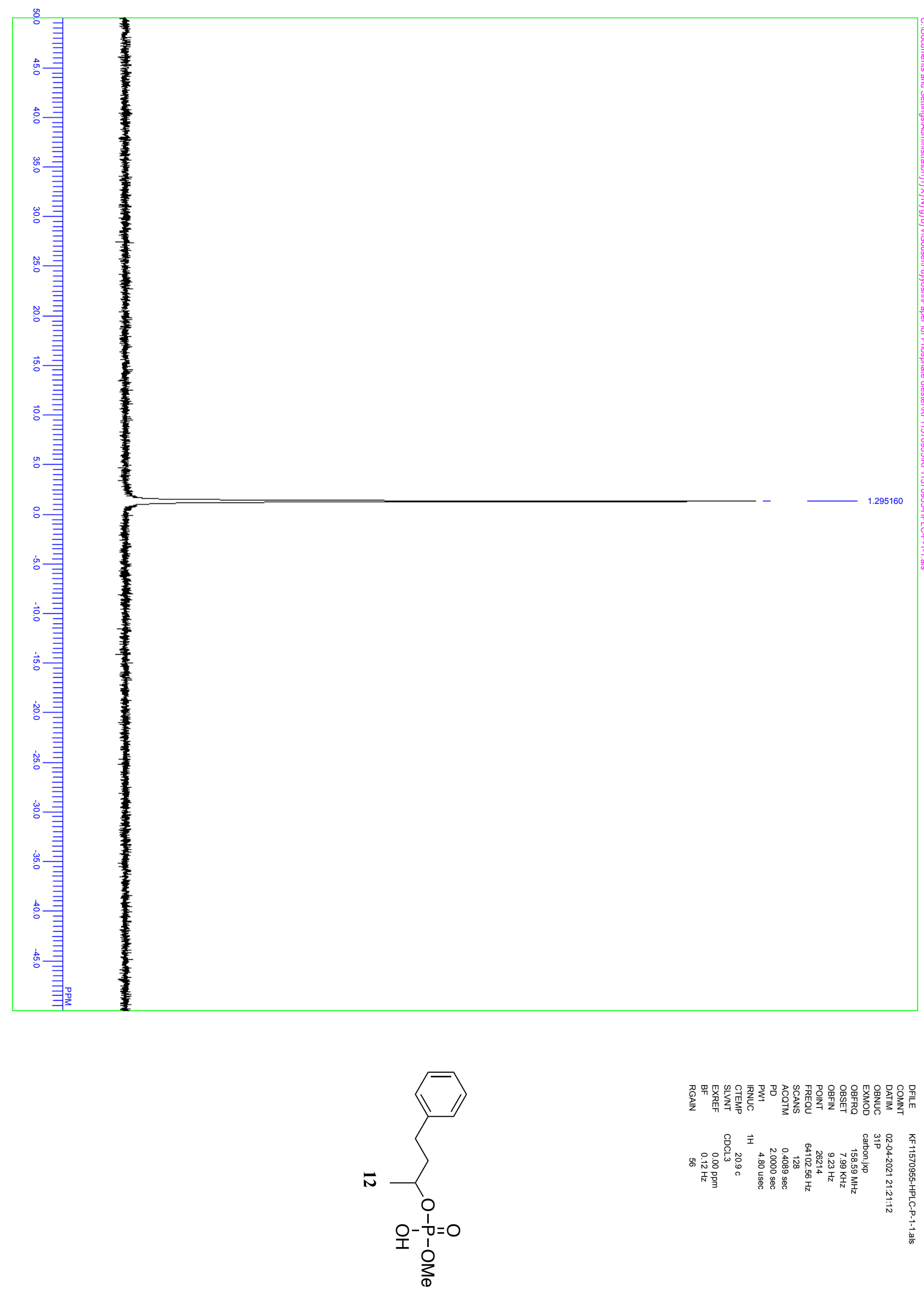

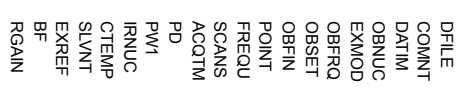

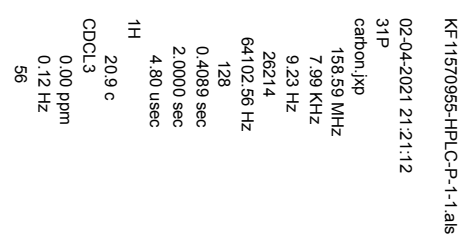



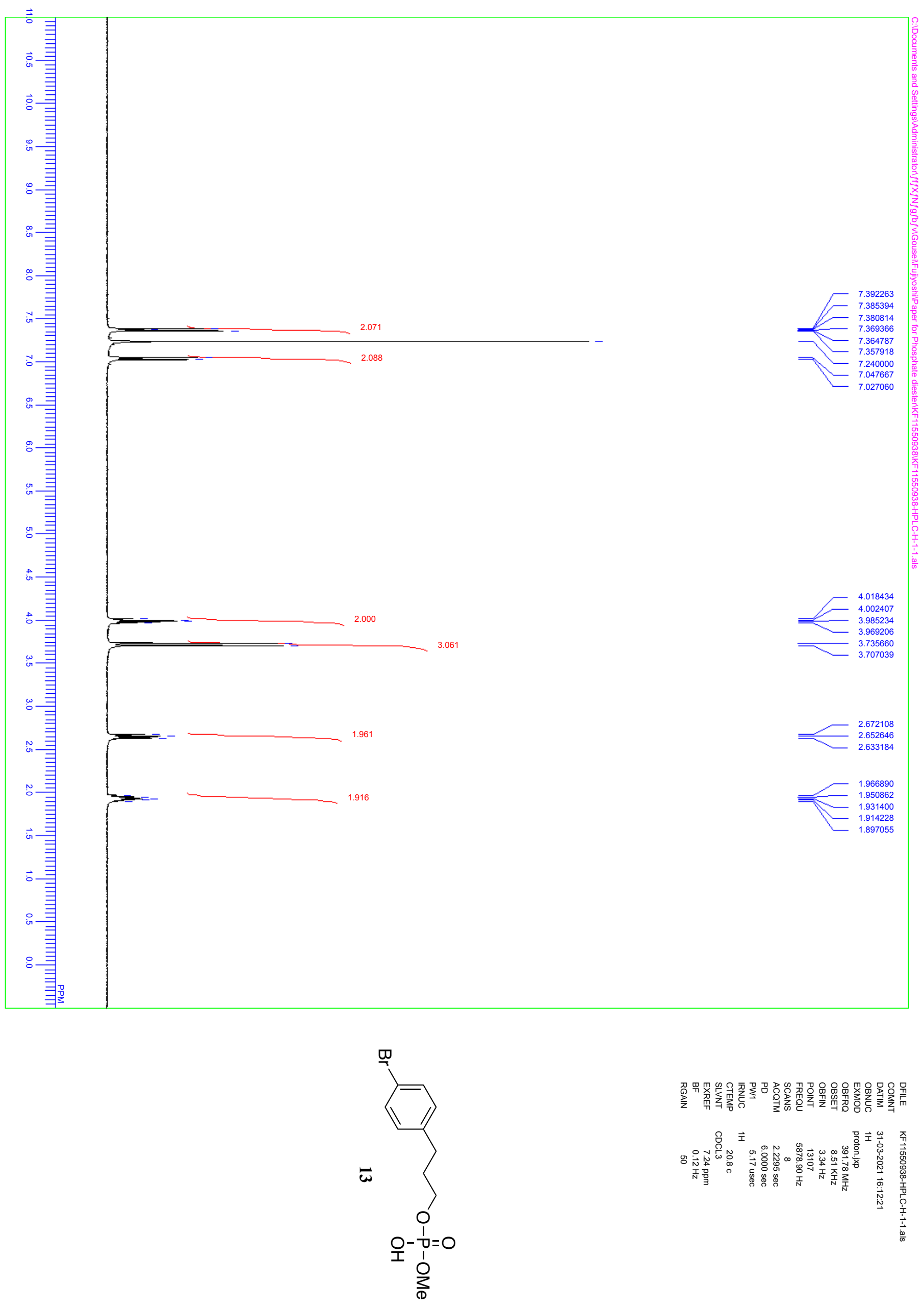

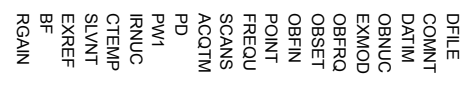

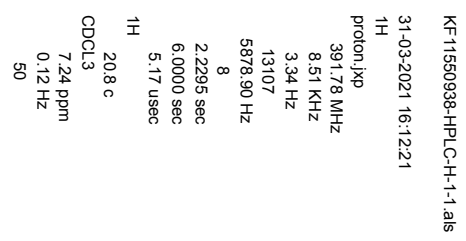



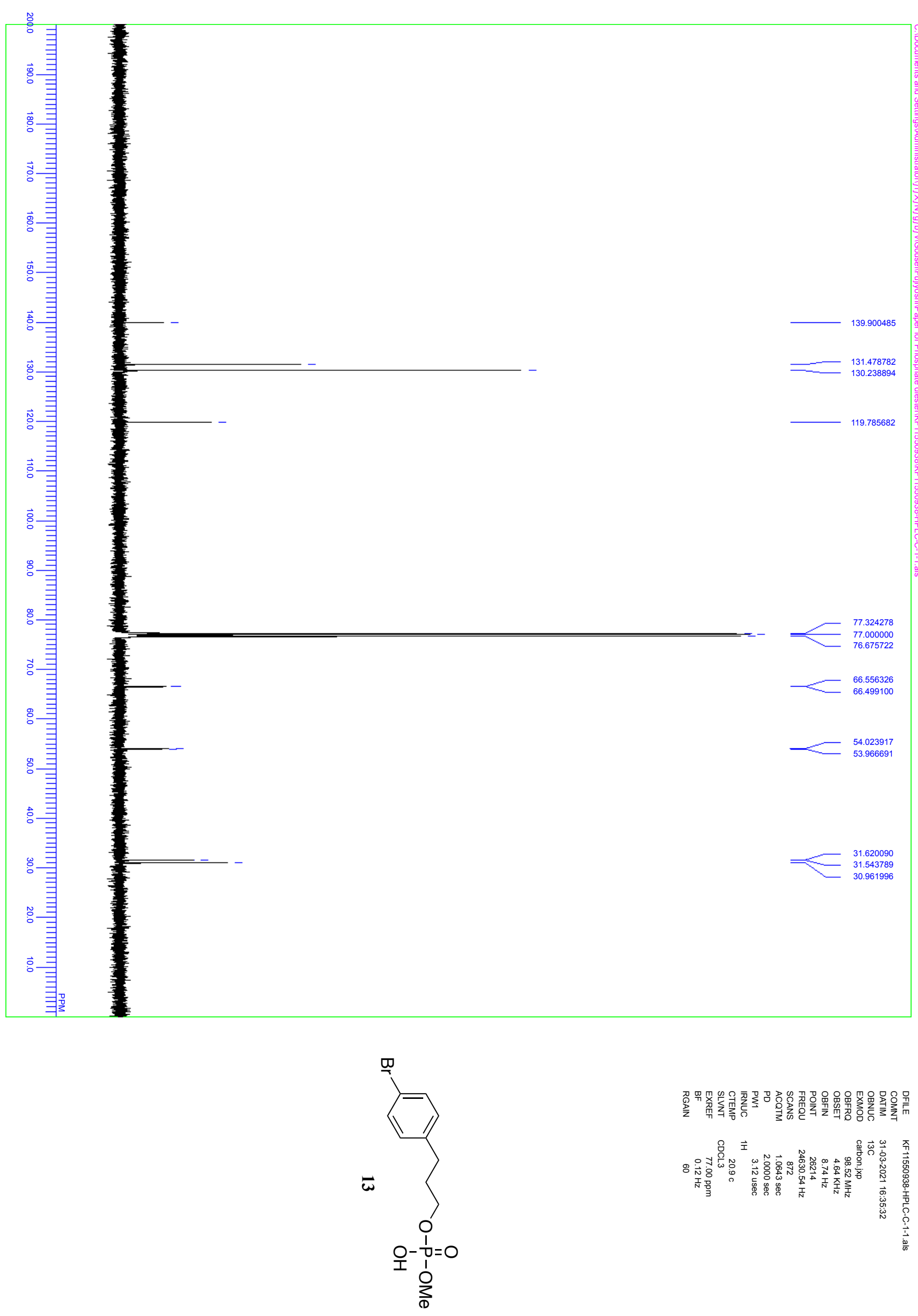

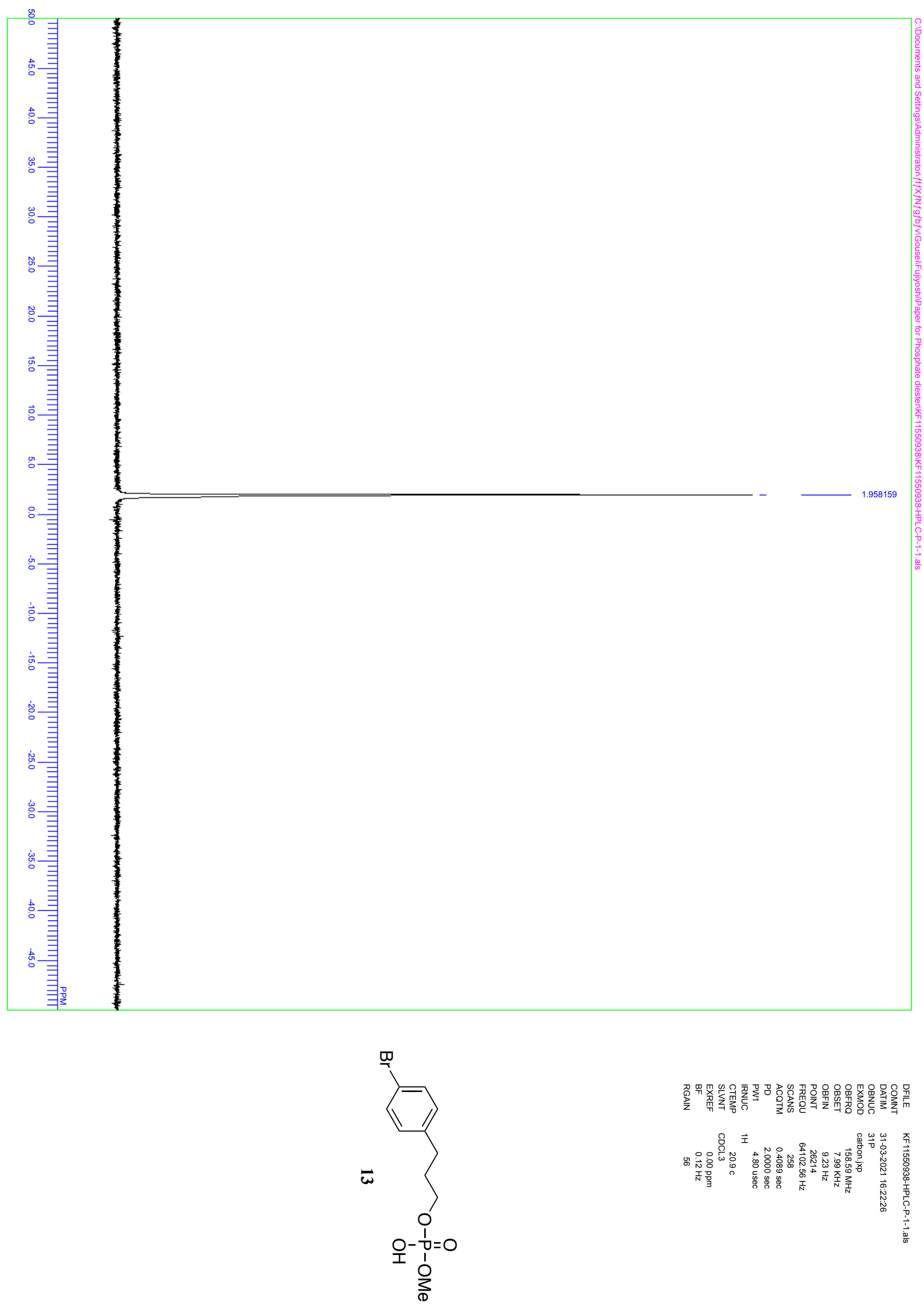

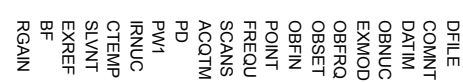

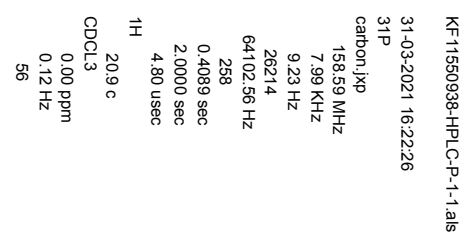



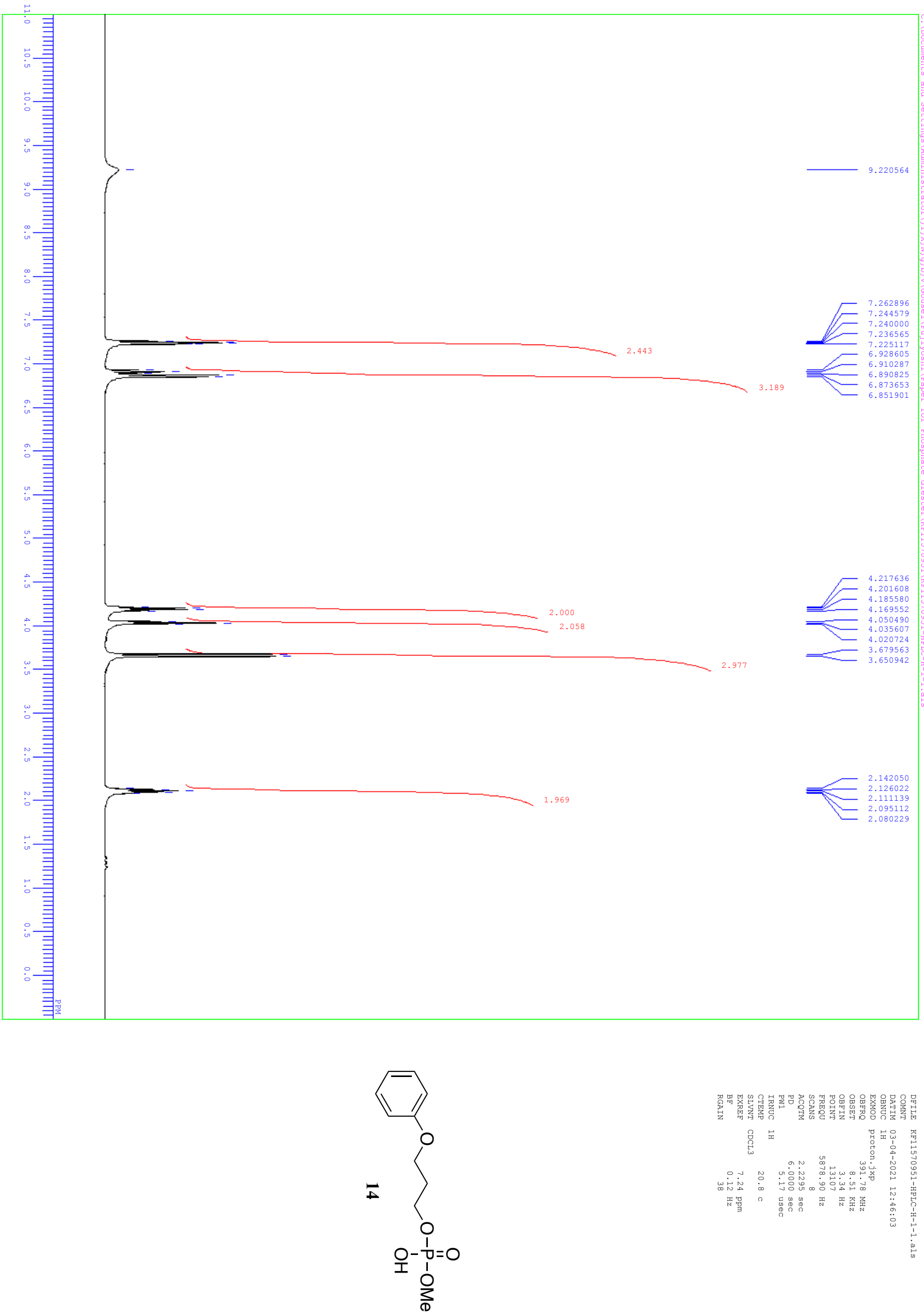

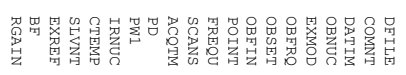

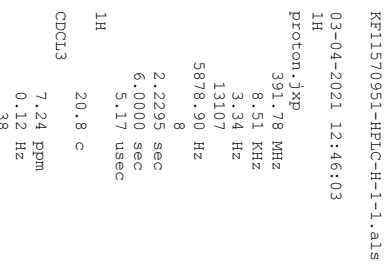



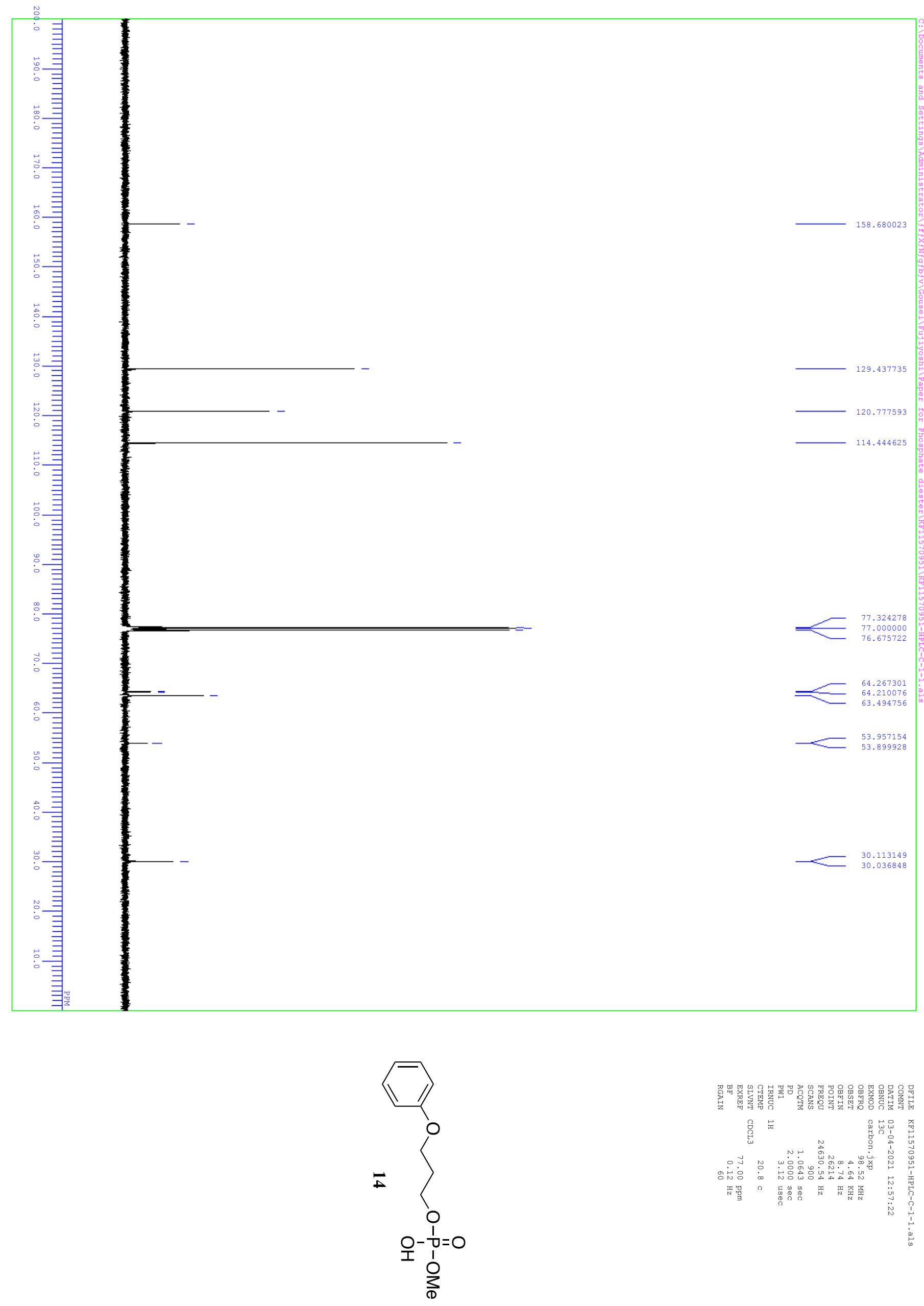

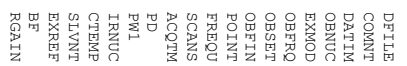

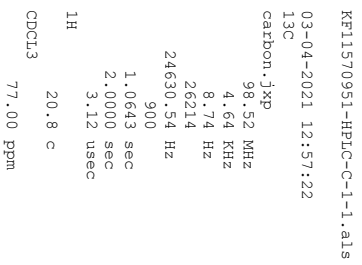




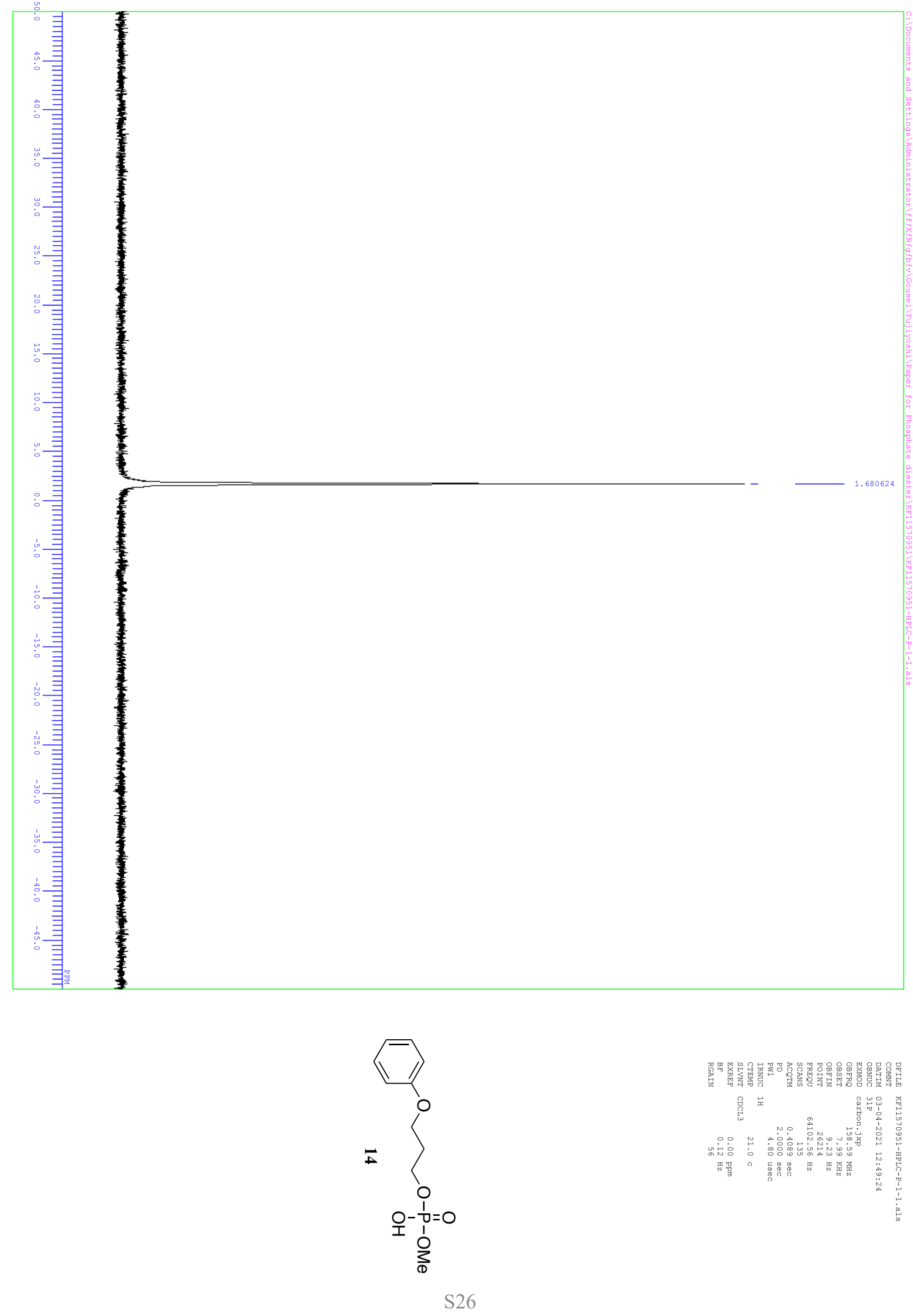



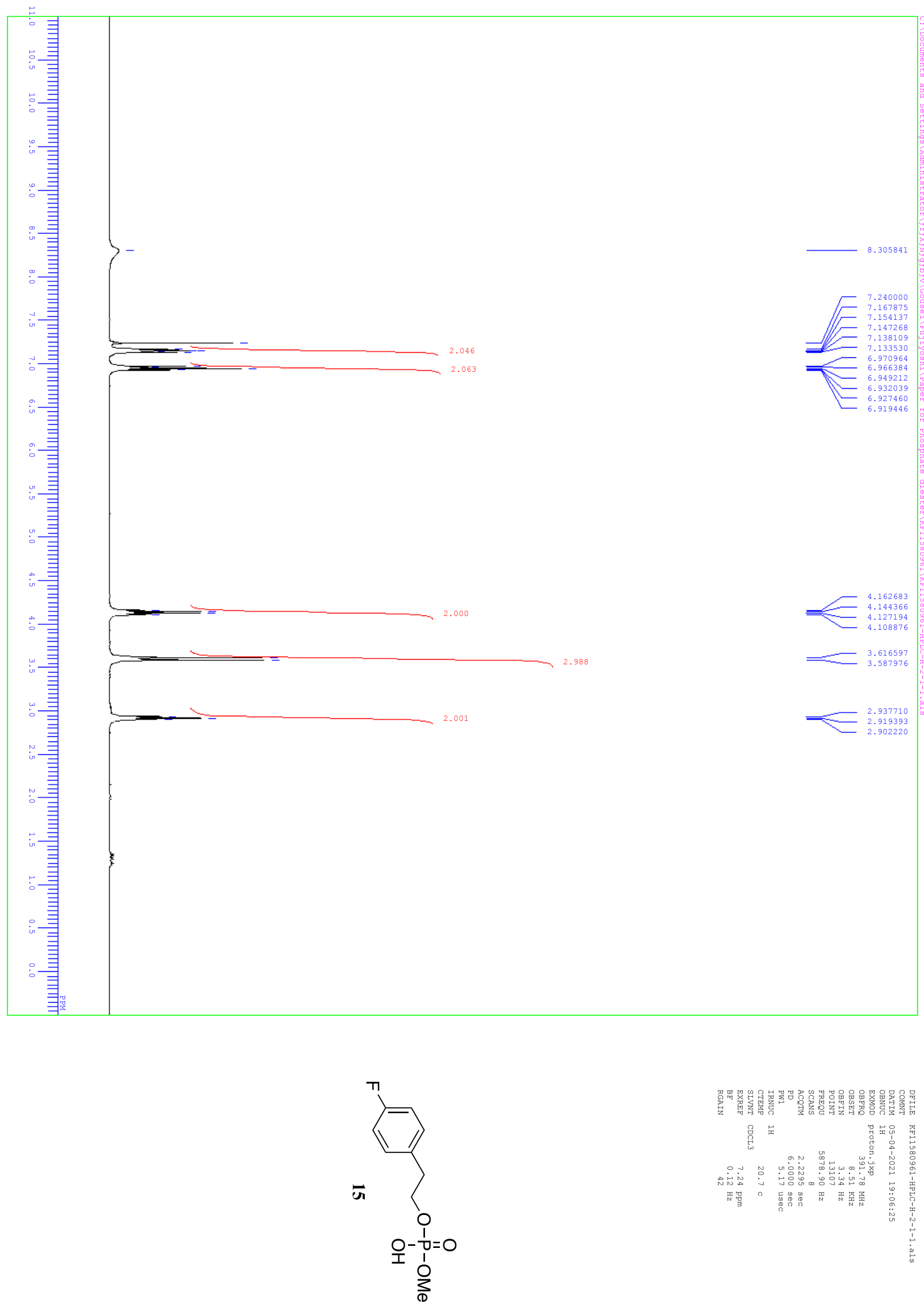

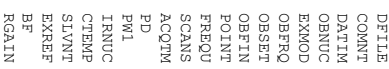
욜

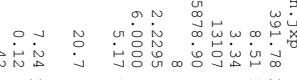

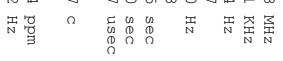



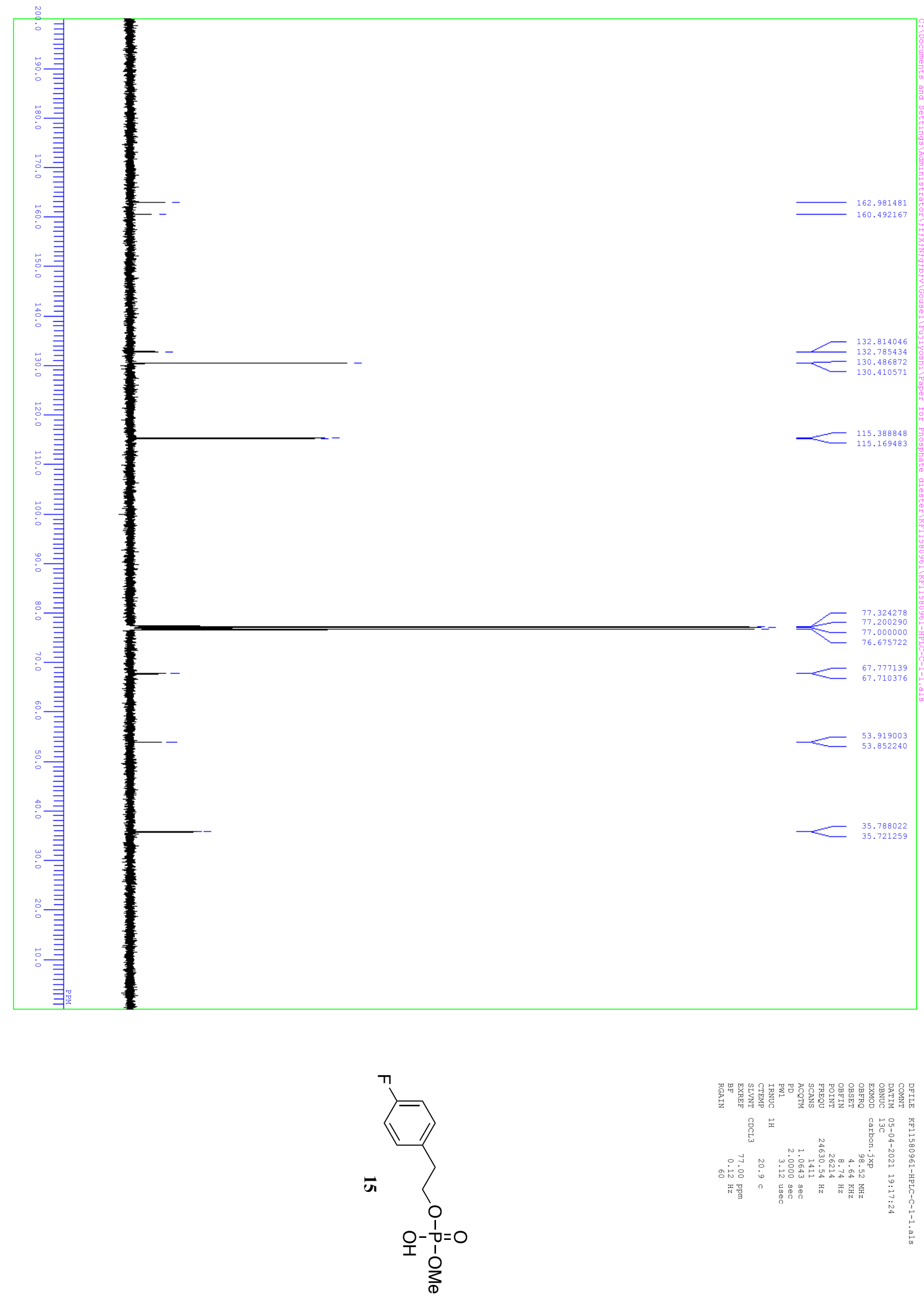

倌"

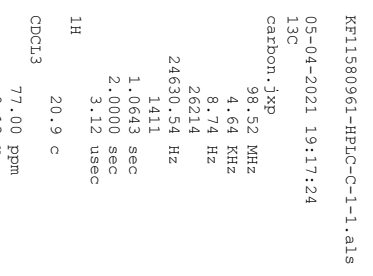




$$
\mid
$$



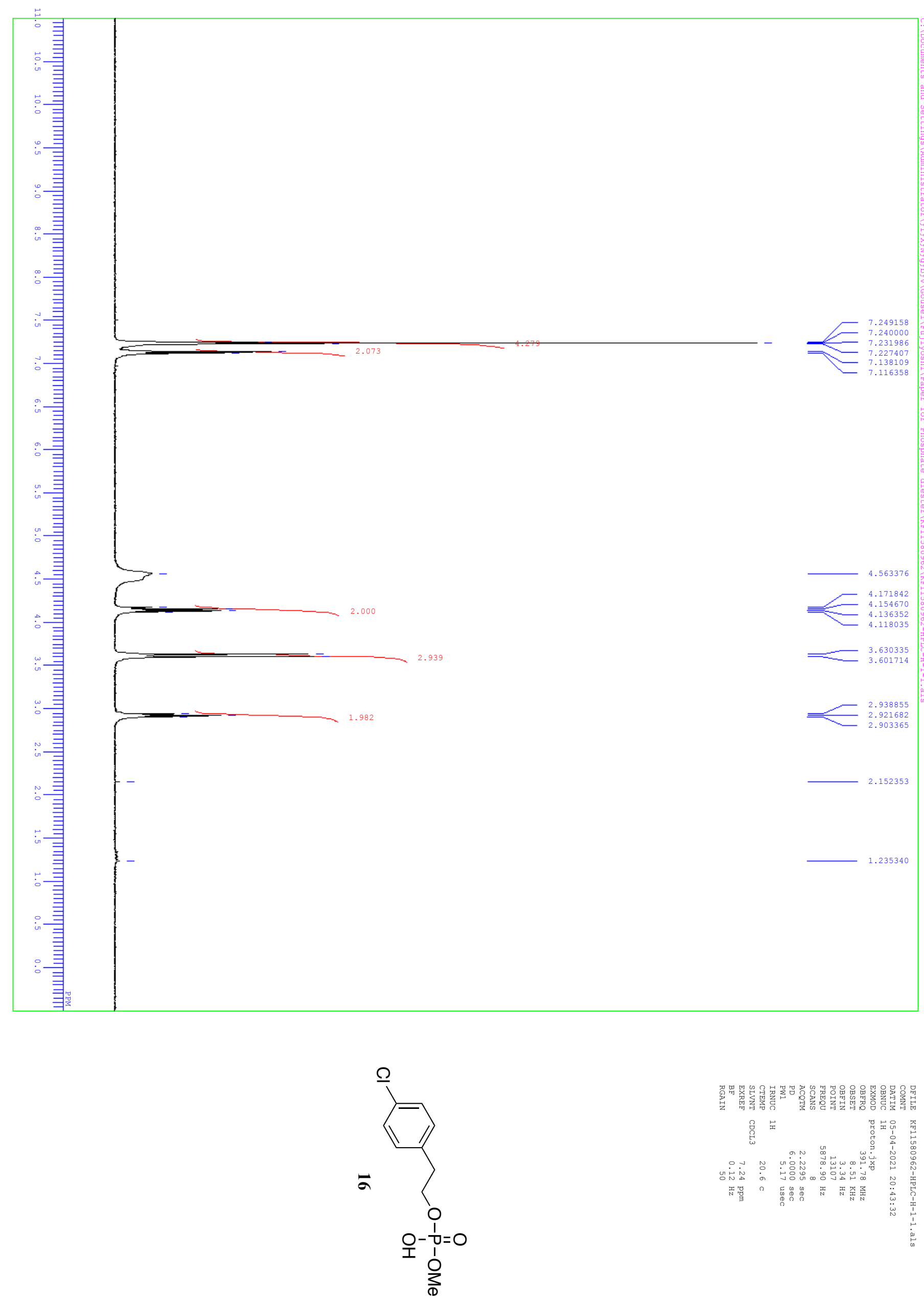

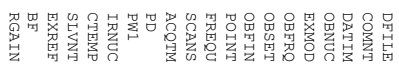

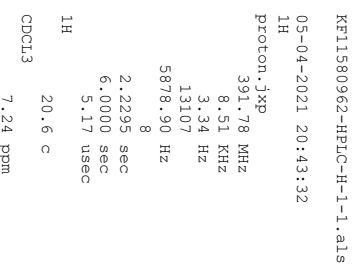



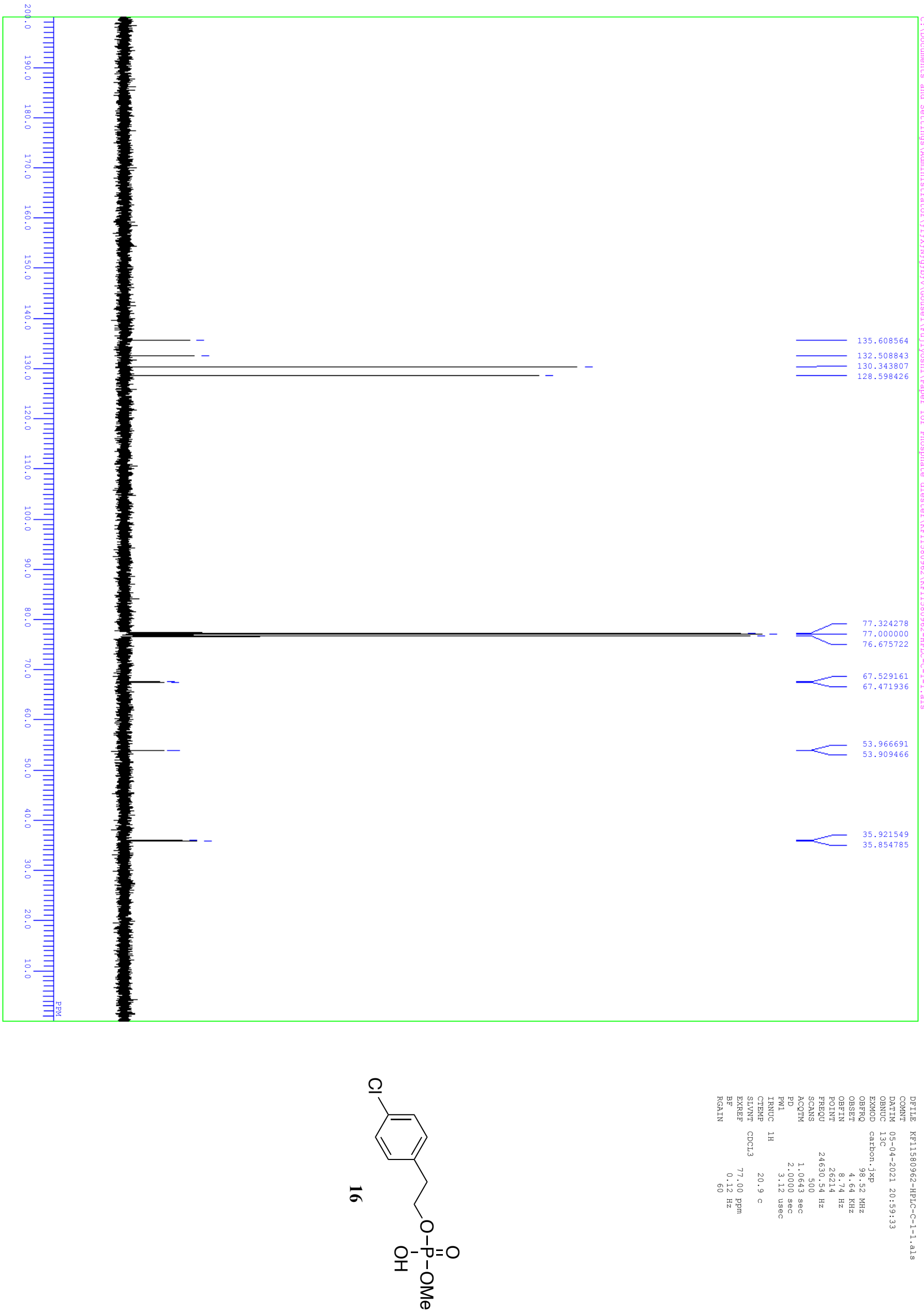

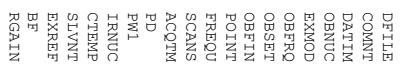

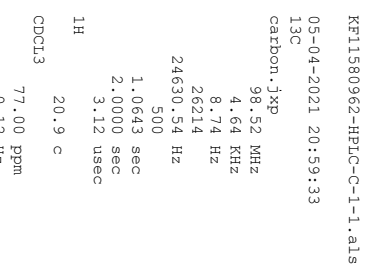




$$
\mid
$$



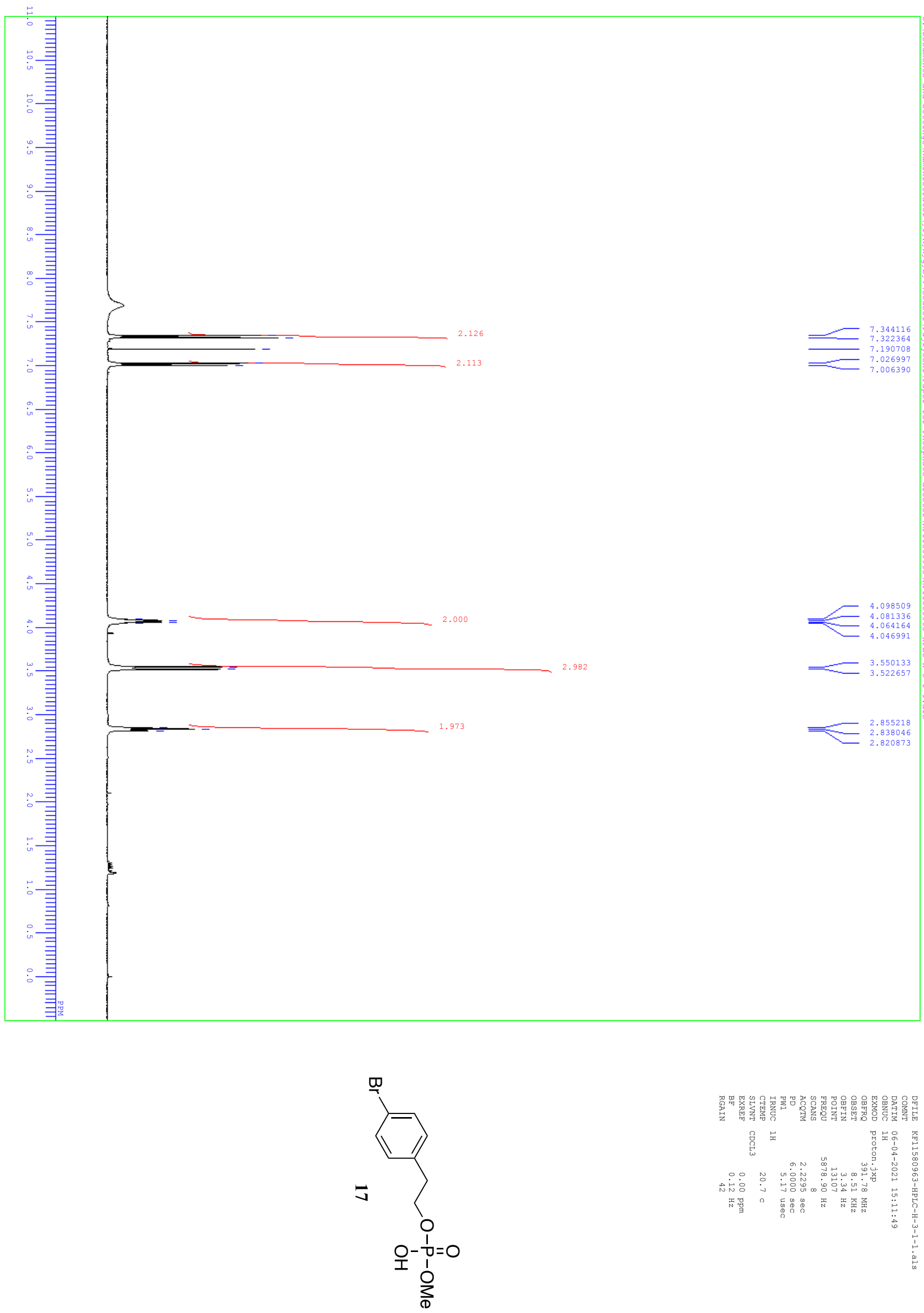

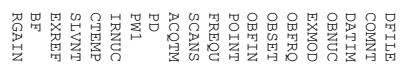
呑岳

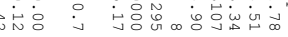

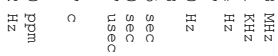



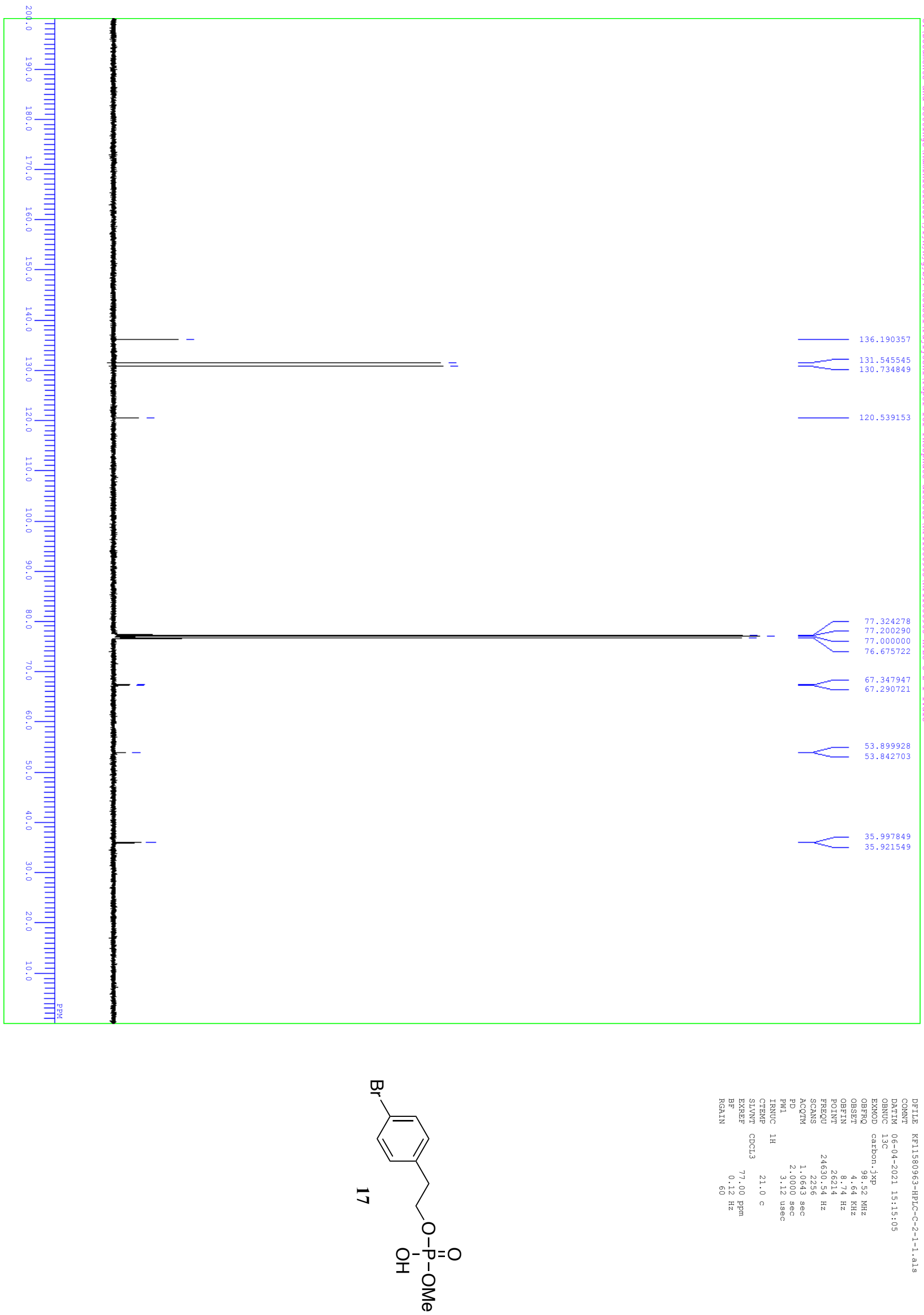

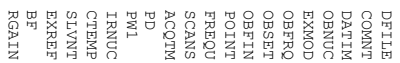

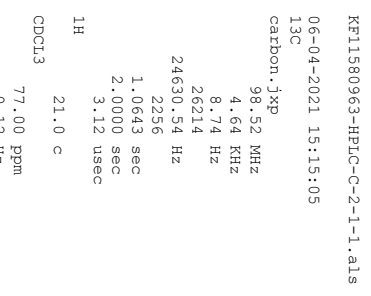




$$
\mid
$$



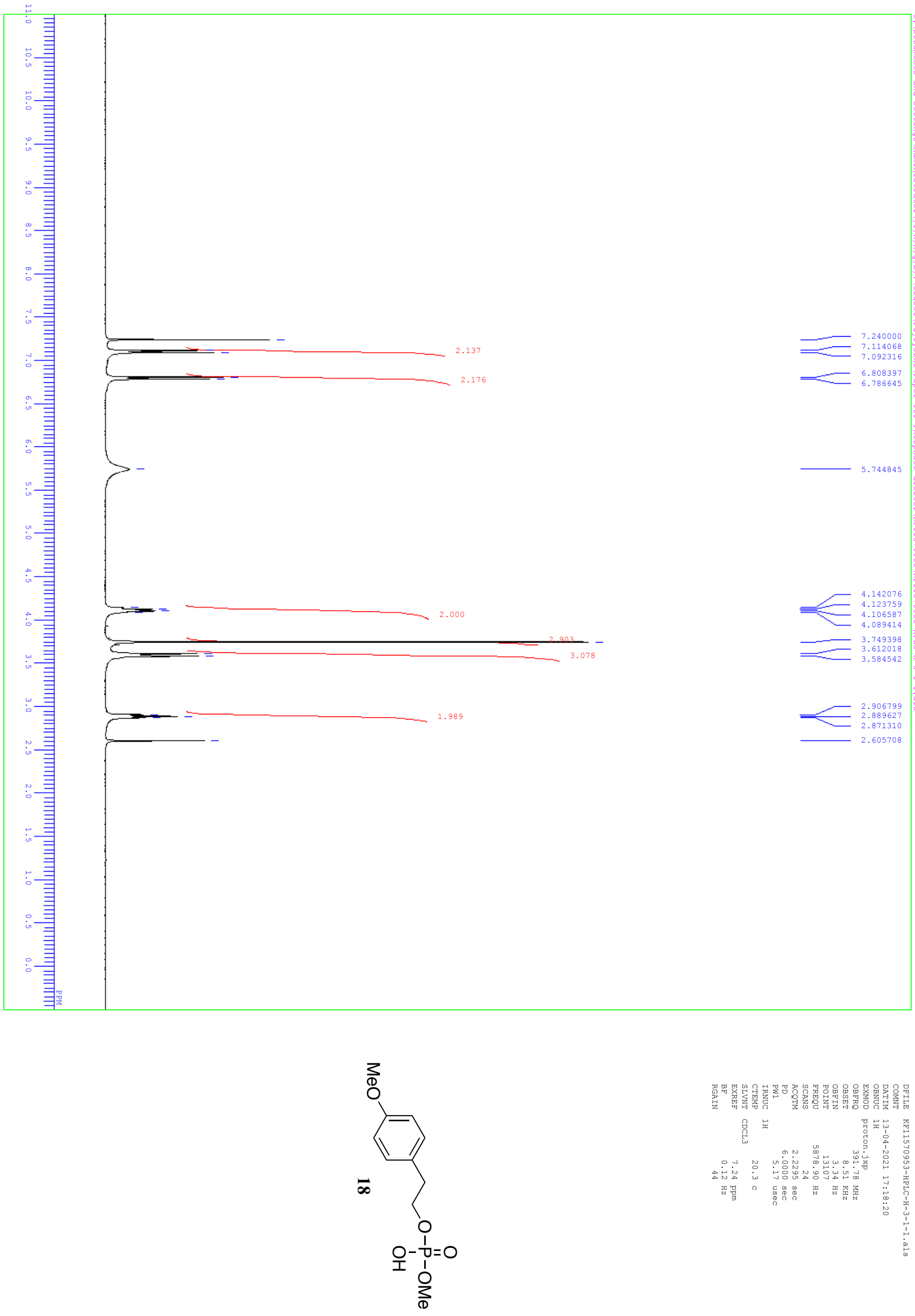

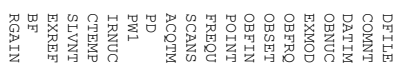

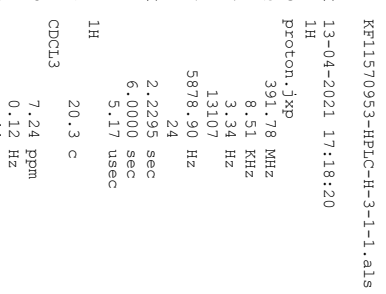




$$
\mid
$$



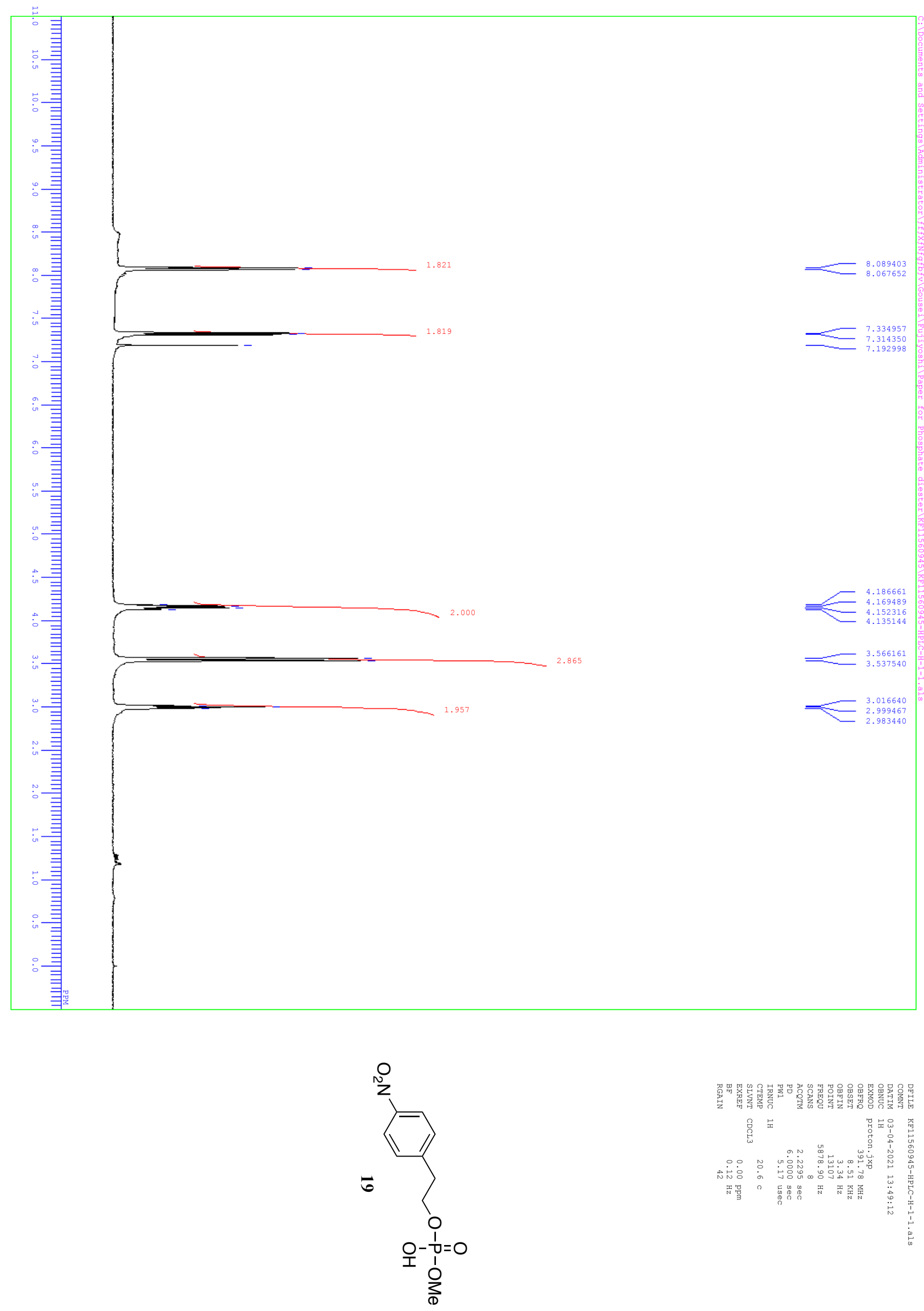

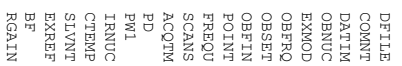
욜

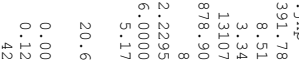

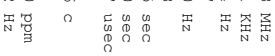



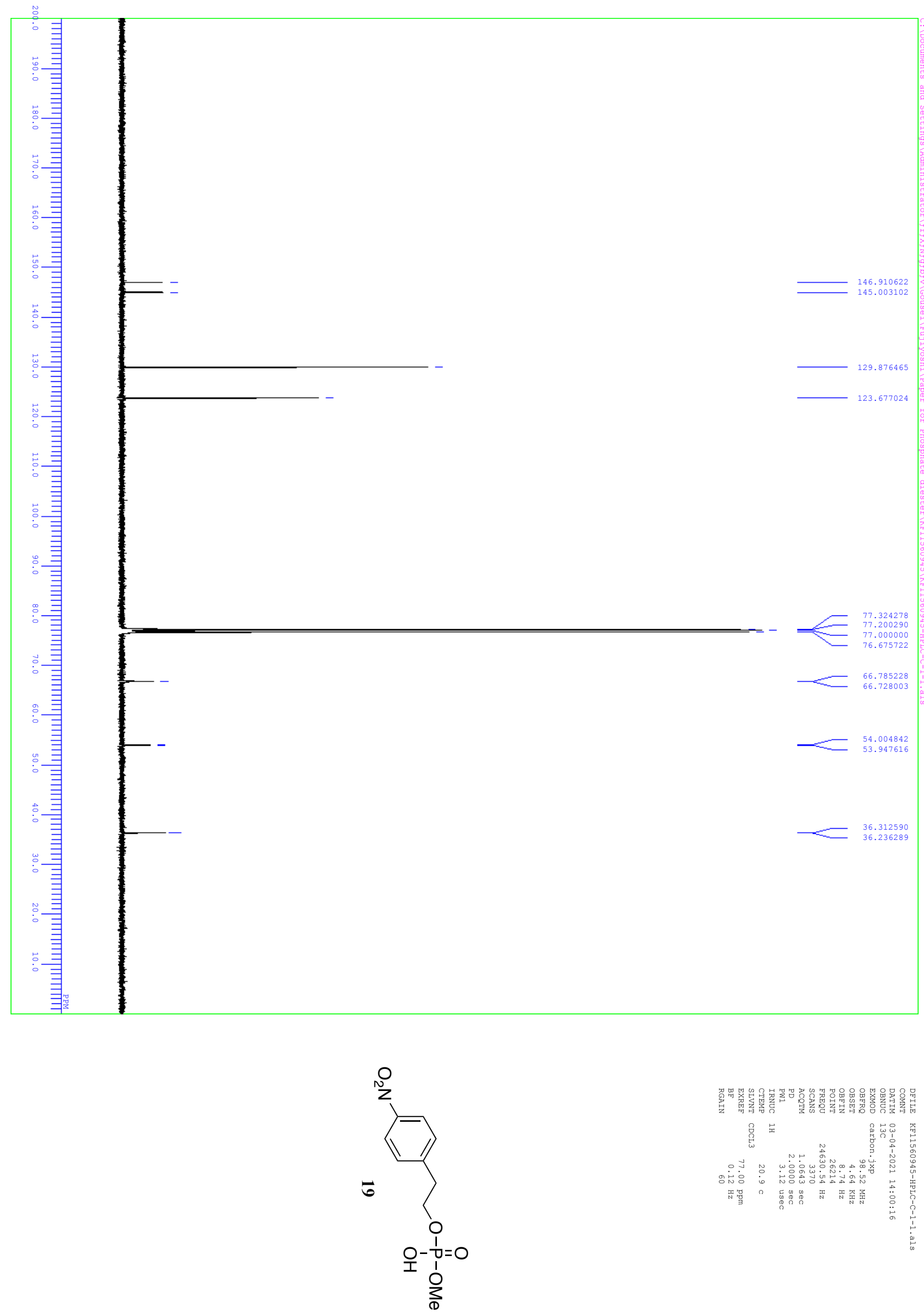

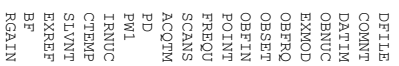
象年 


$$
F
$$



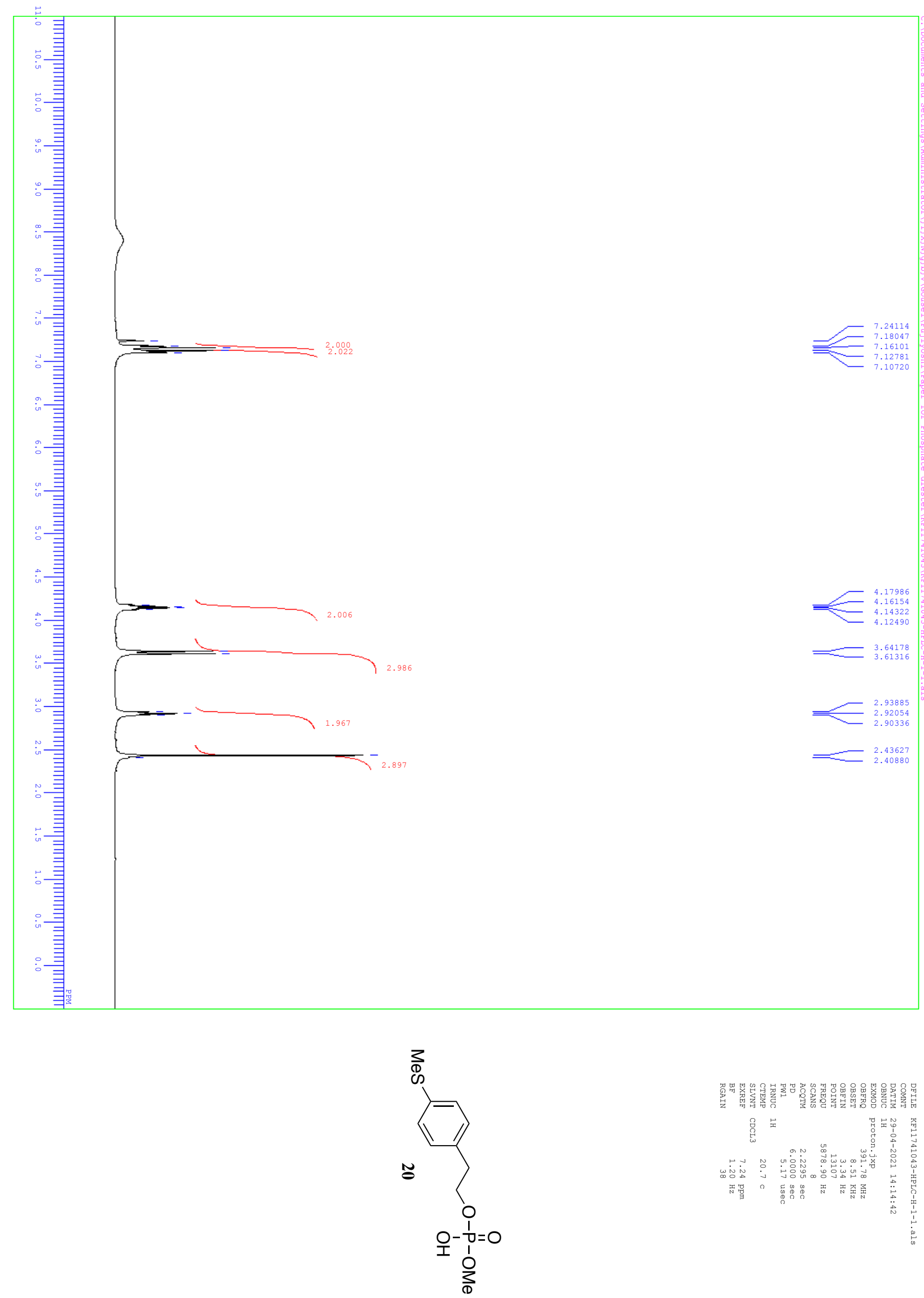

嚁"

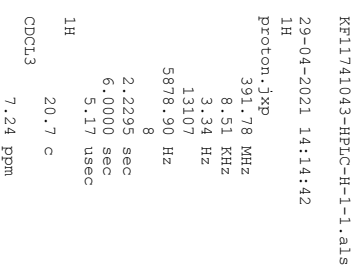



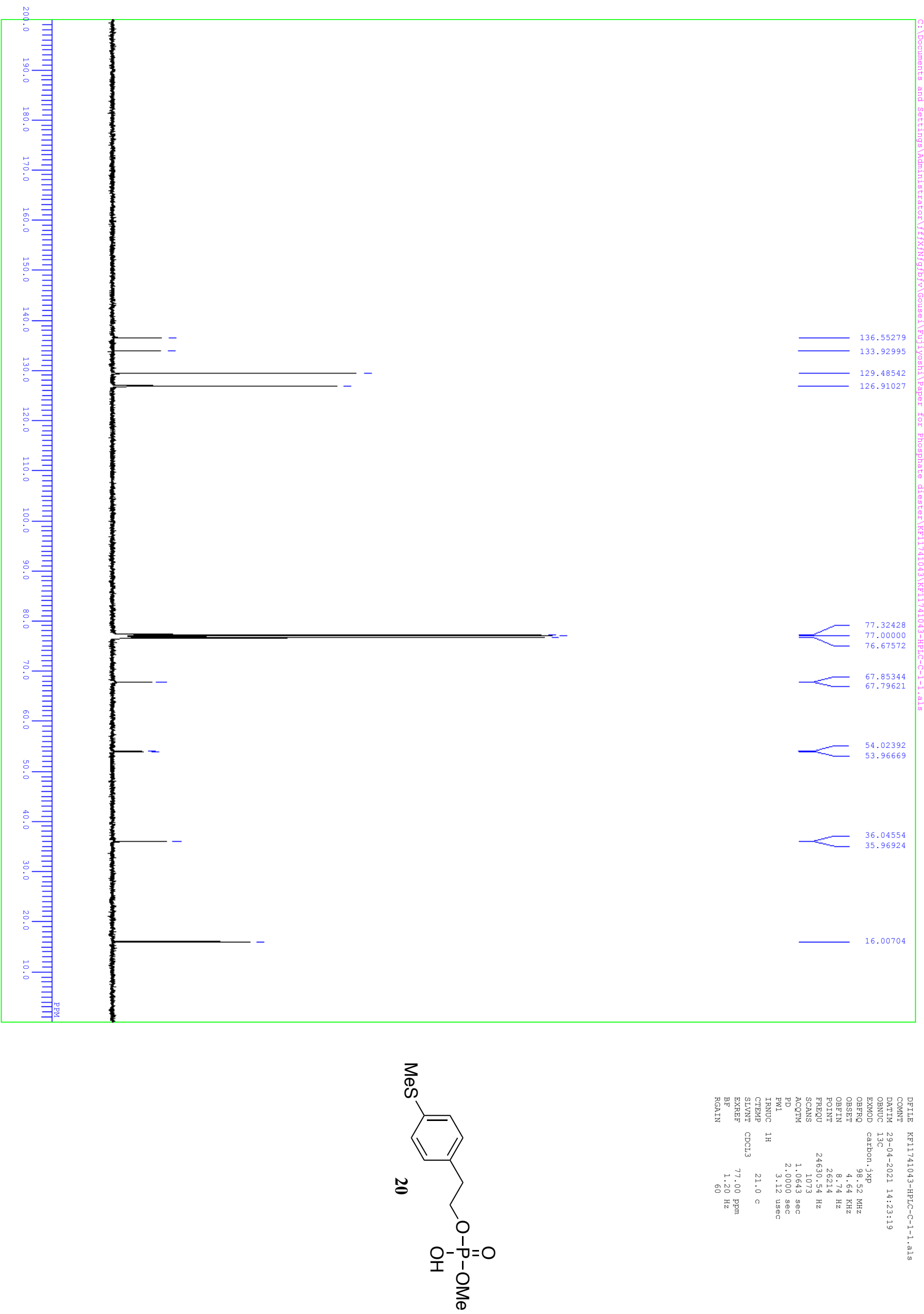

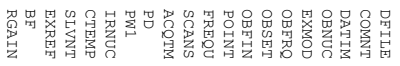
总点

$\begin{array}{rll} & \\ 0\end{array}$ 

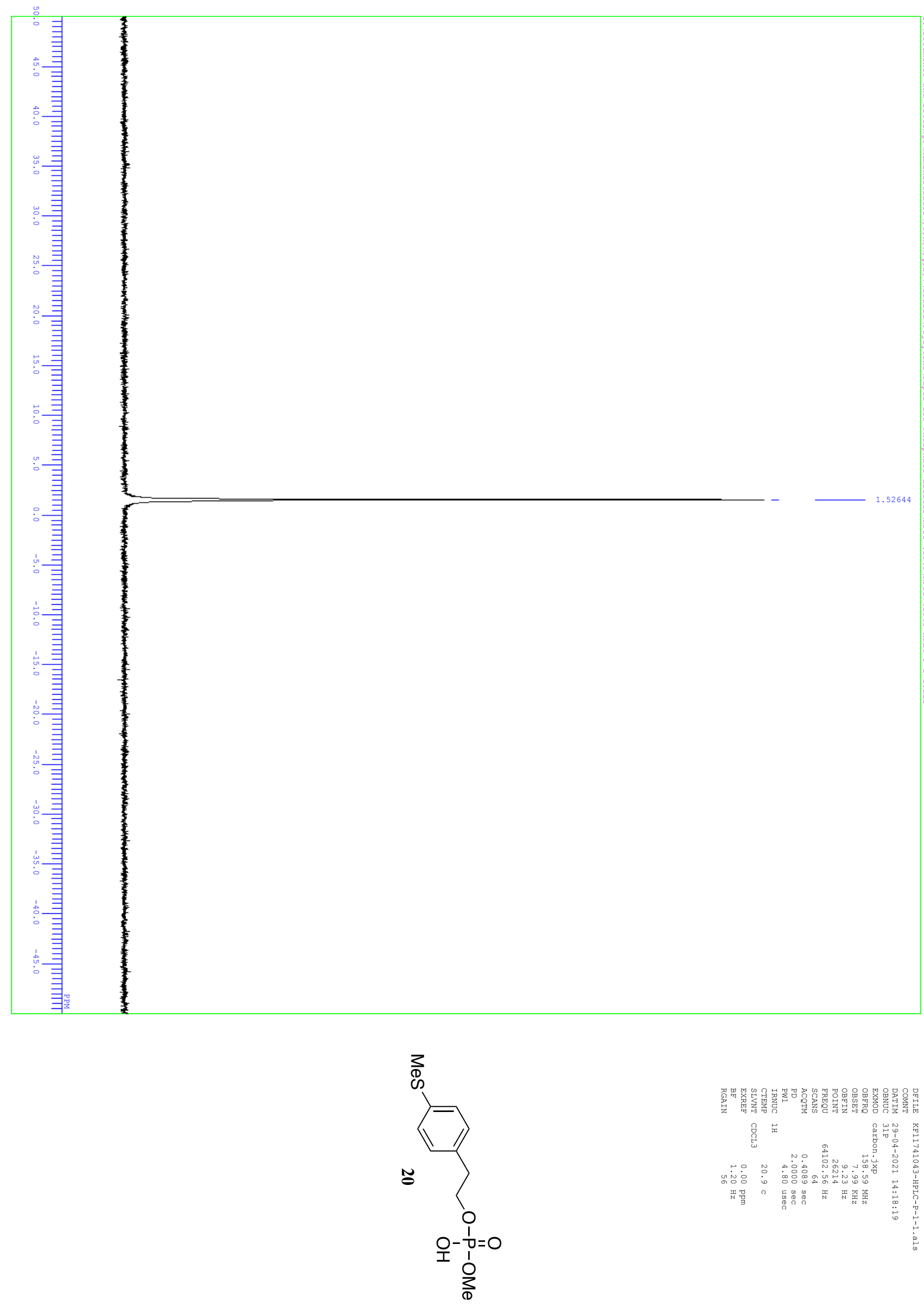

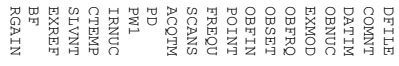

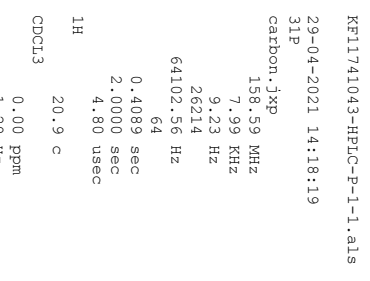




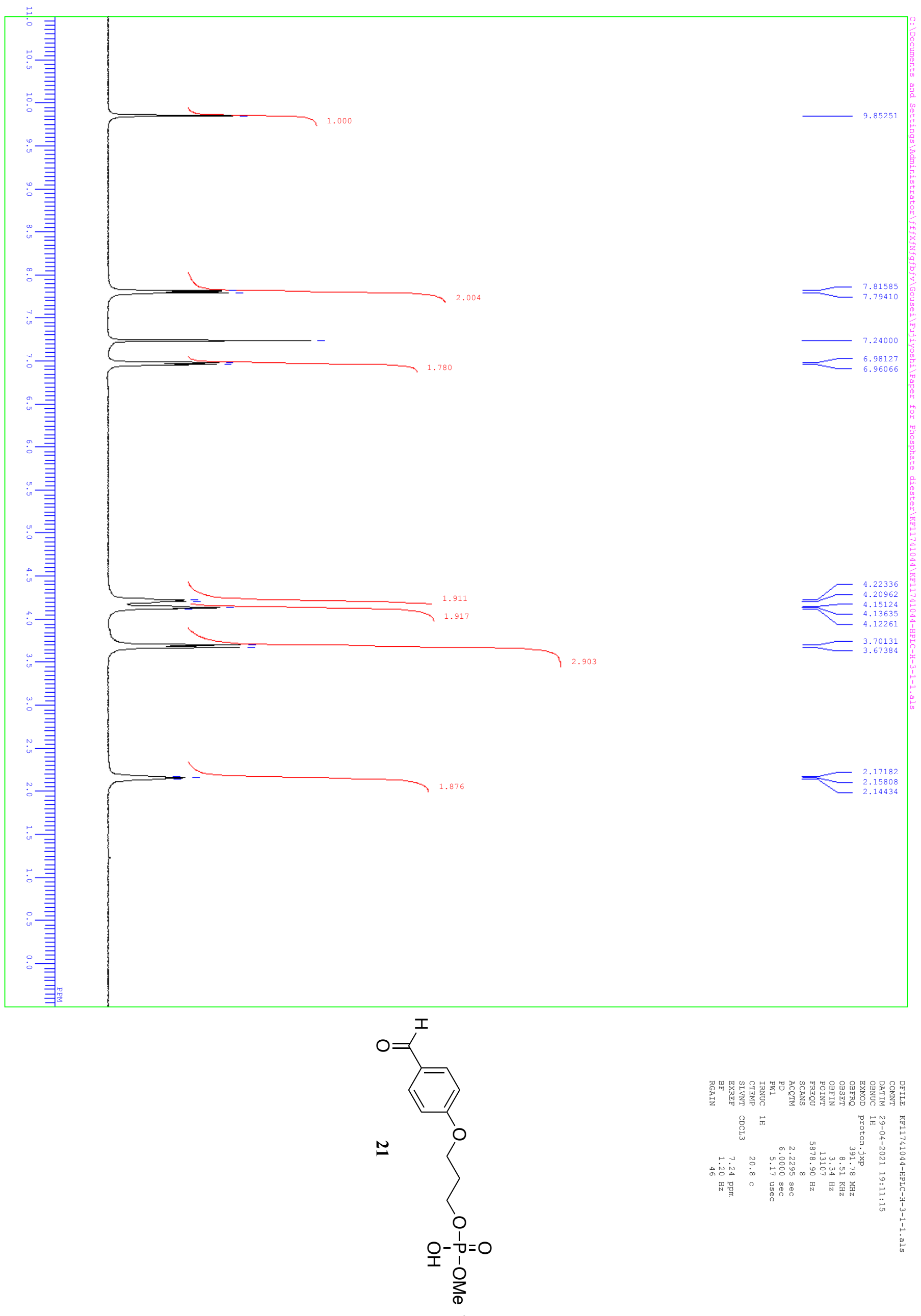




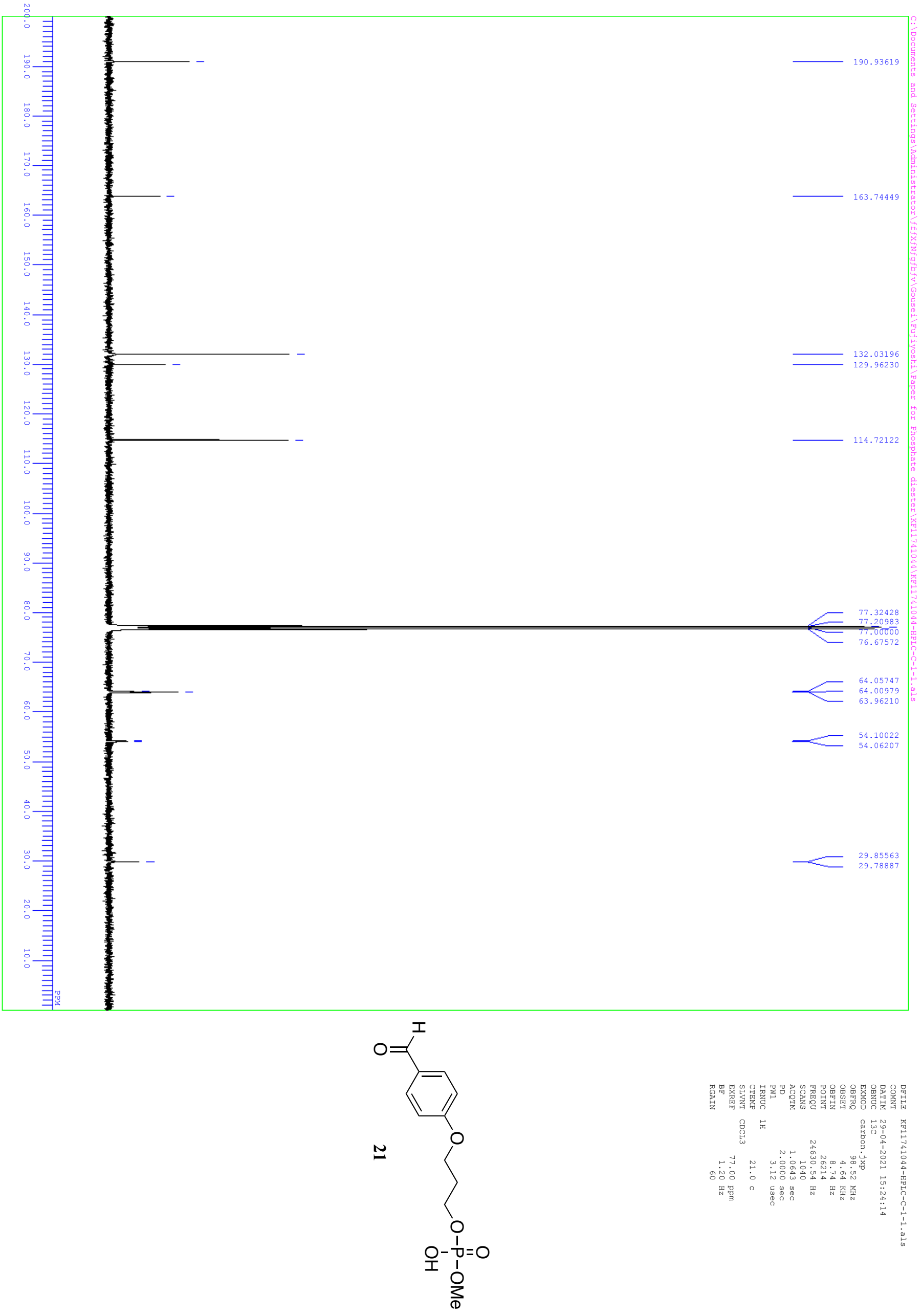




$$
\mid
$$



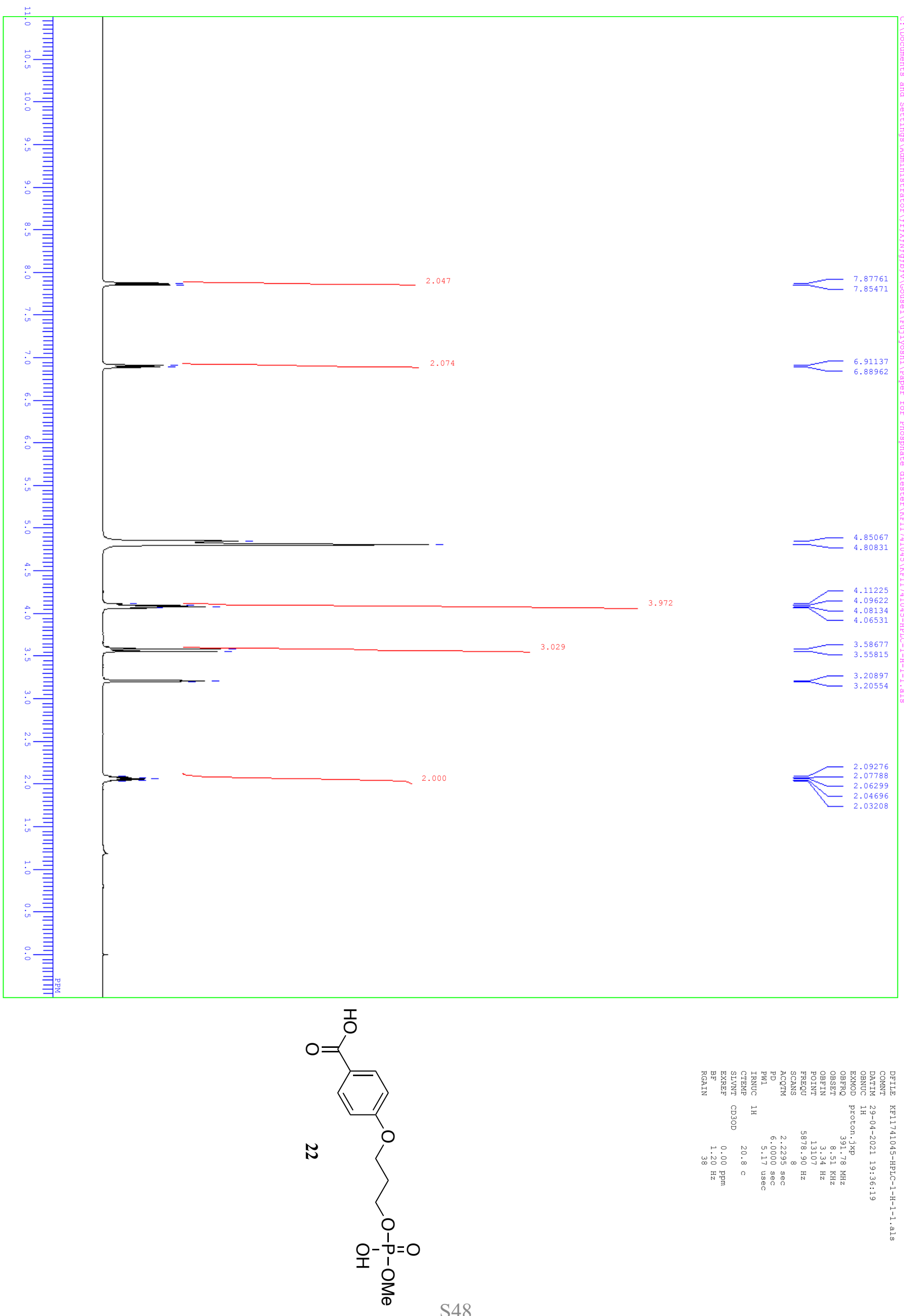

嚁"

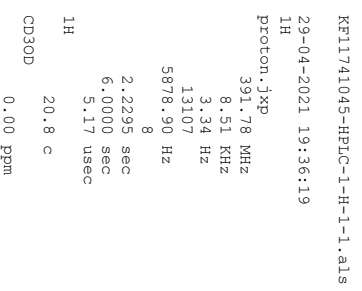



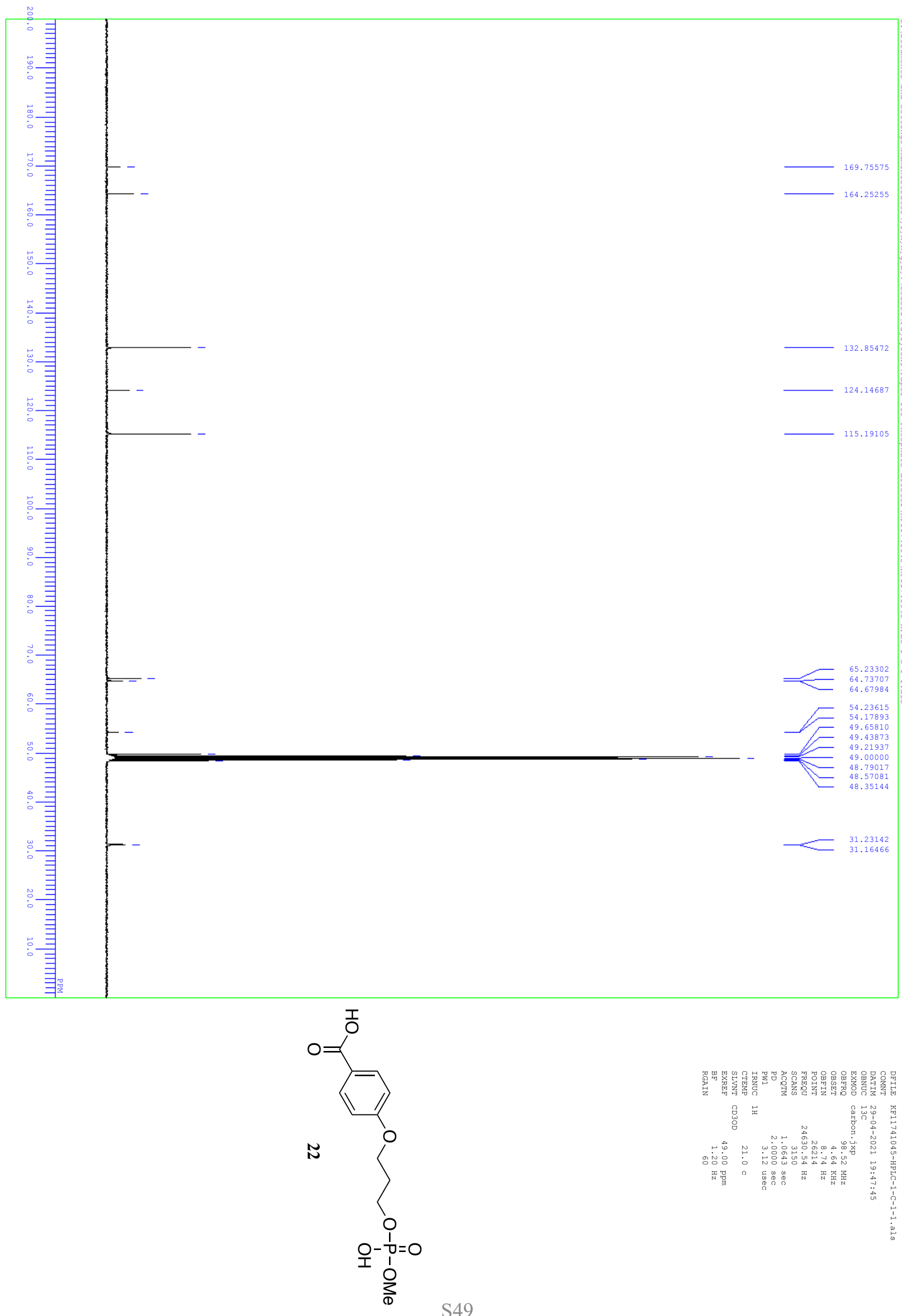

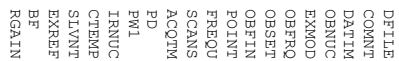

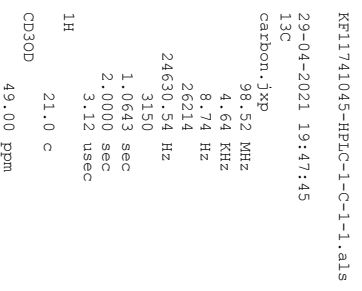




$$
\mid
$$



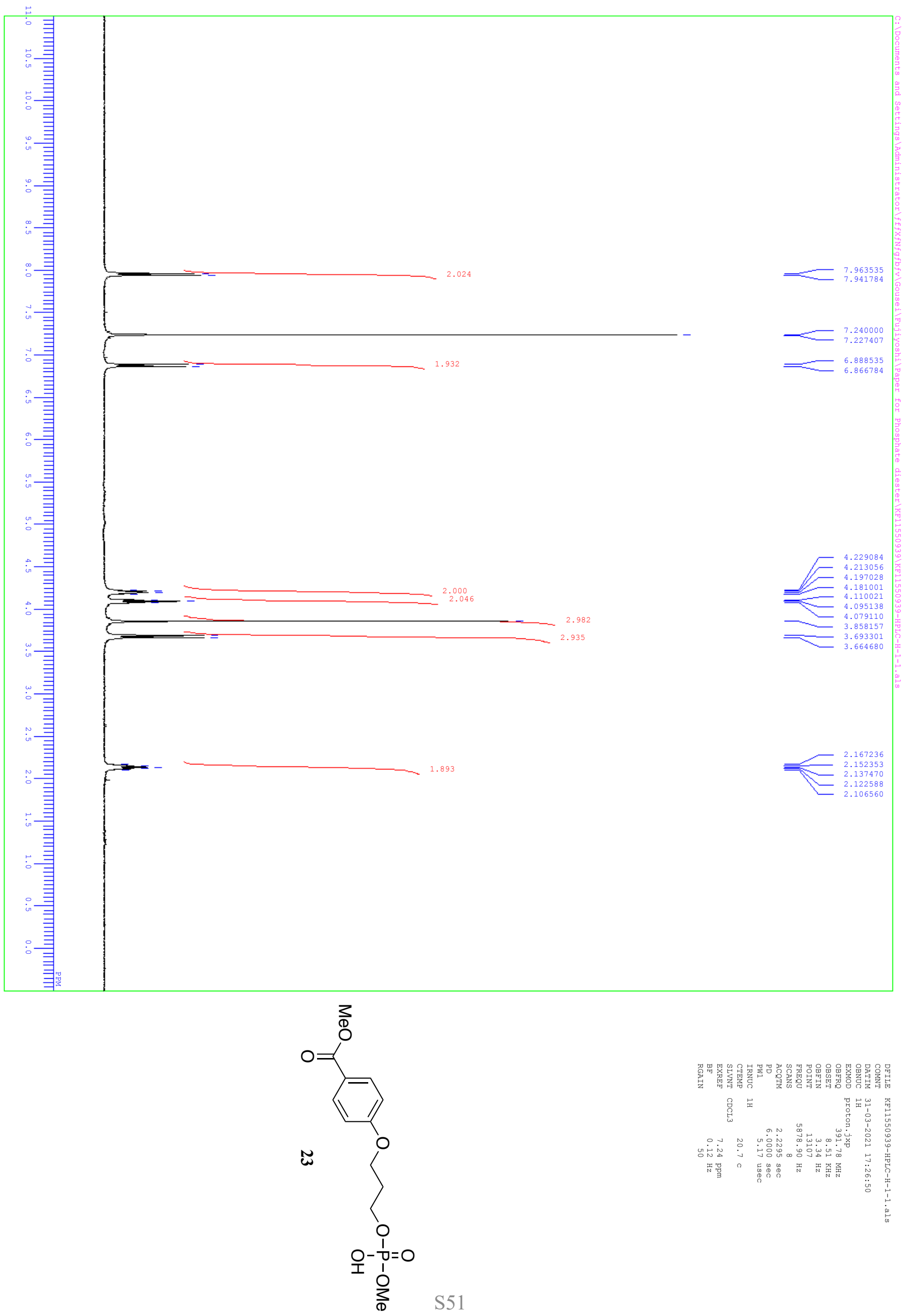

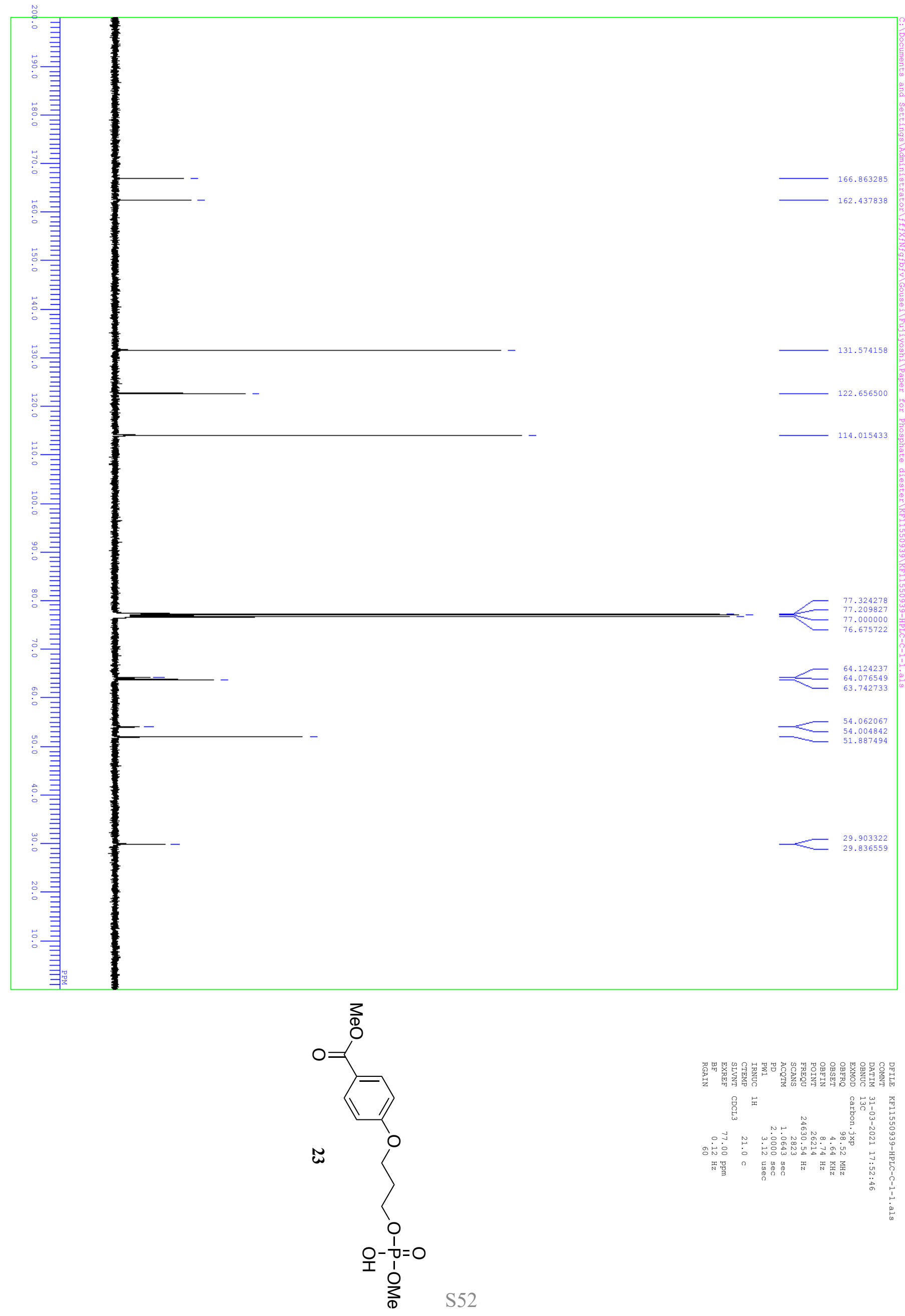

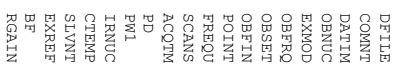

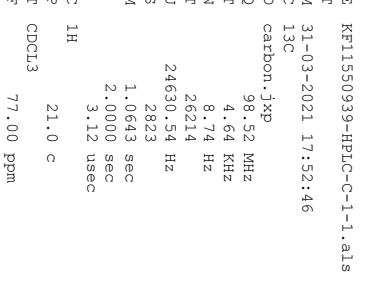




$$
\mid
$$



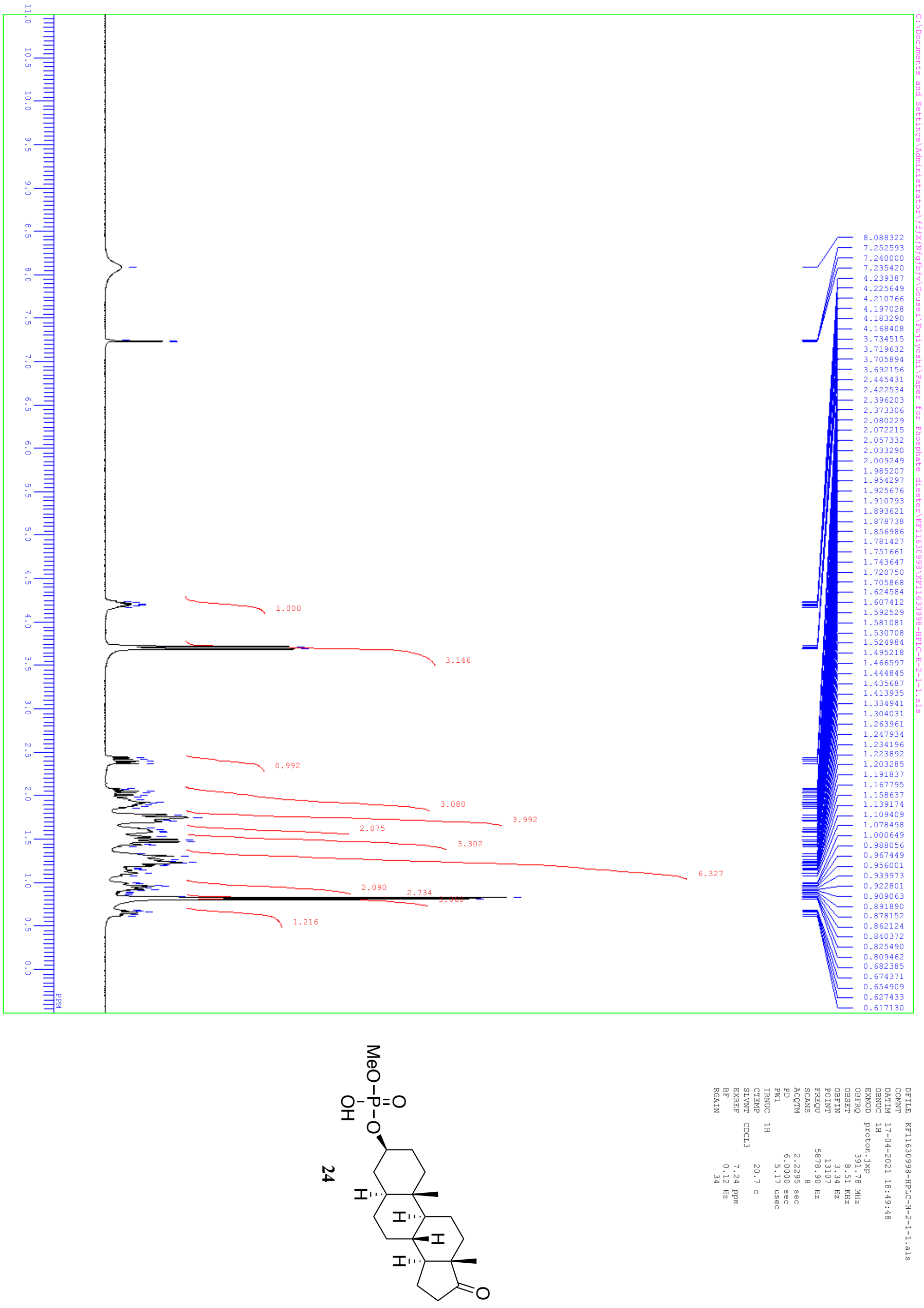

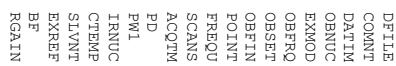

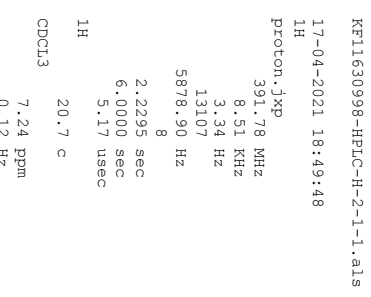



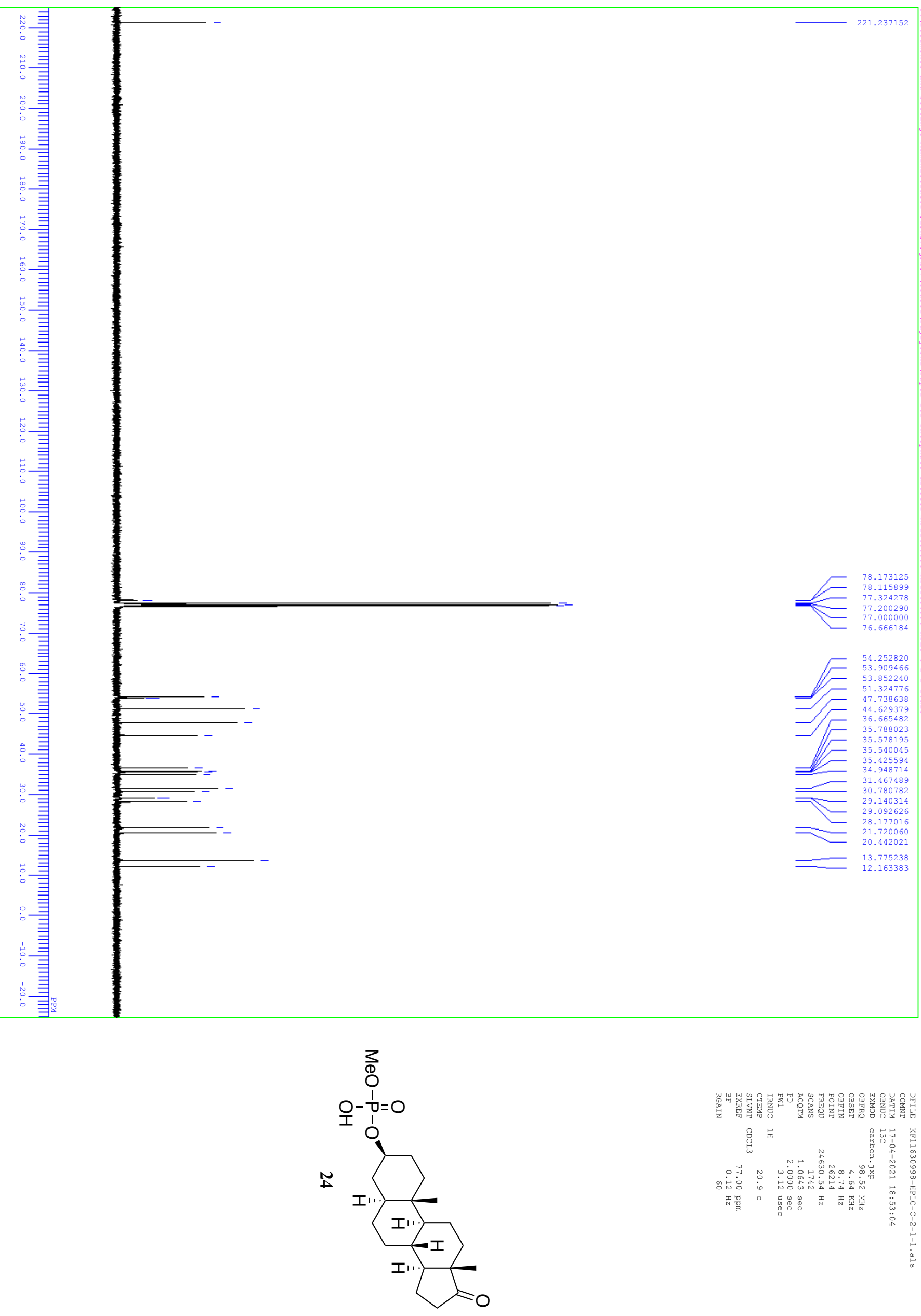

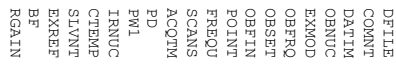

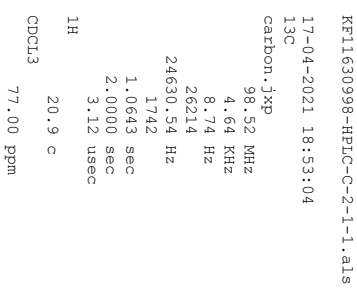



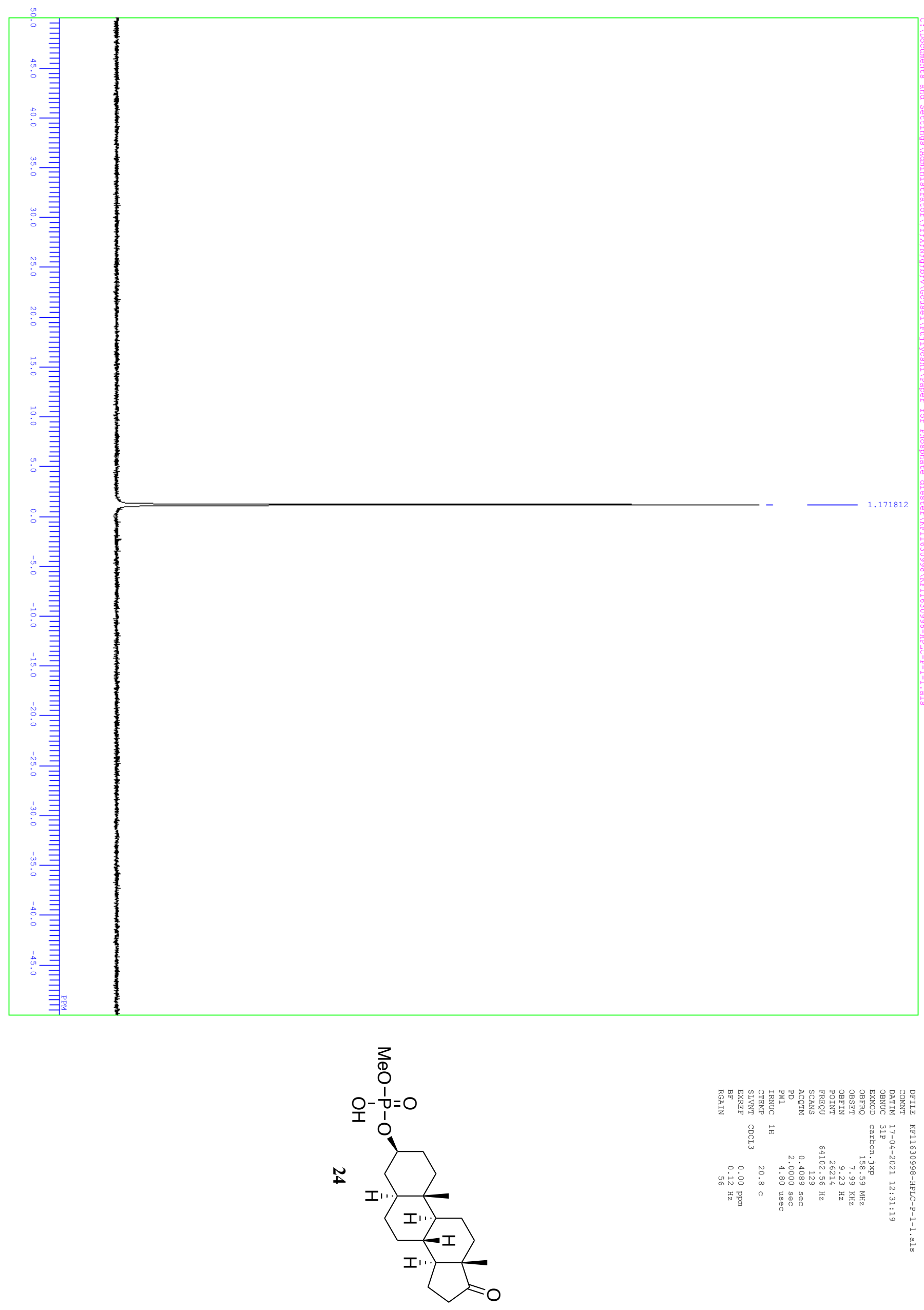

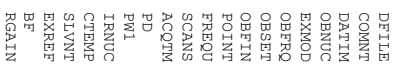
욜

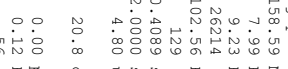

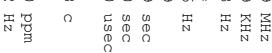



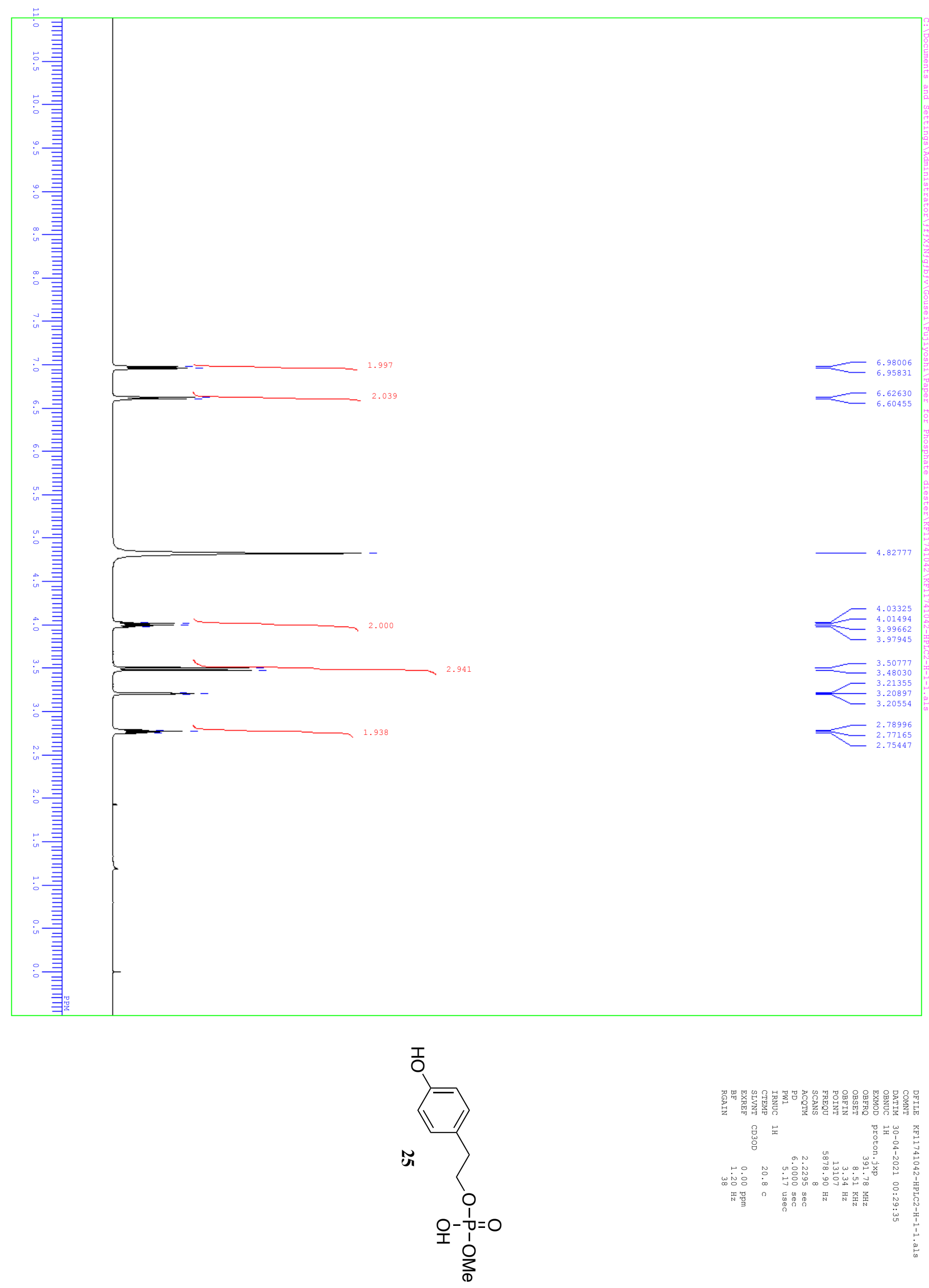

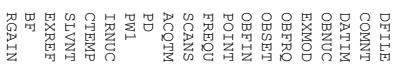
忌

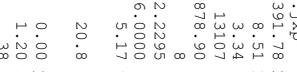

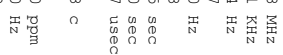



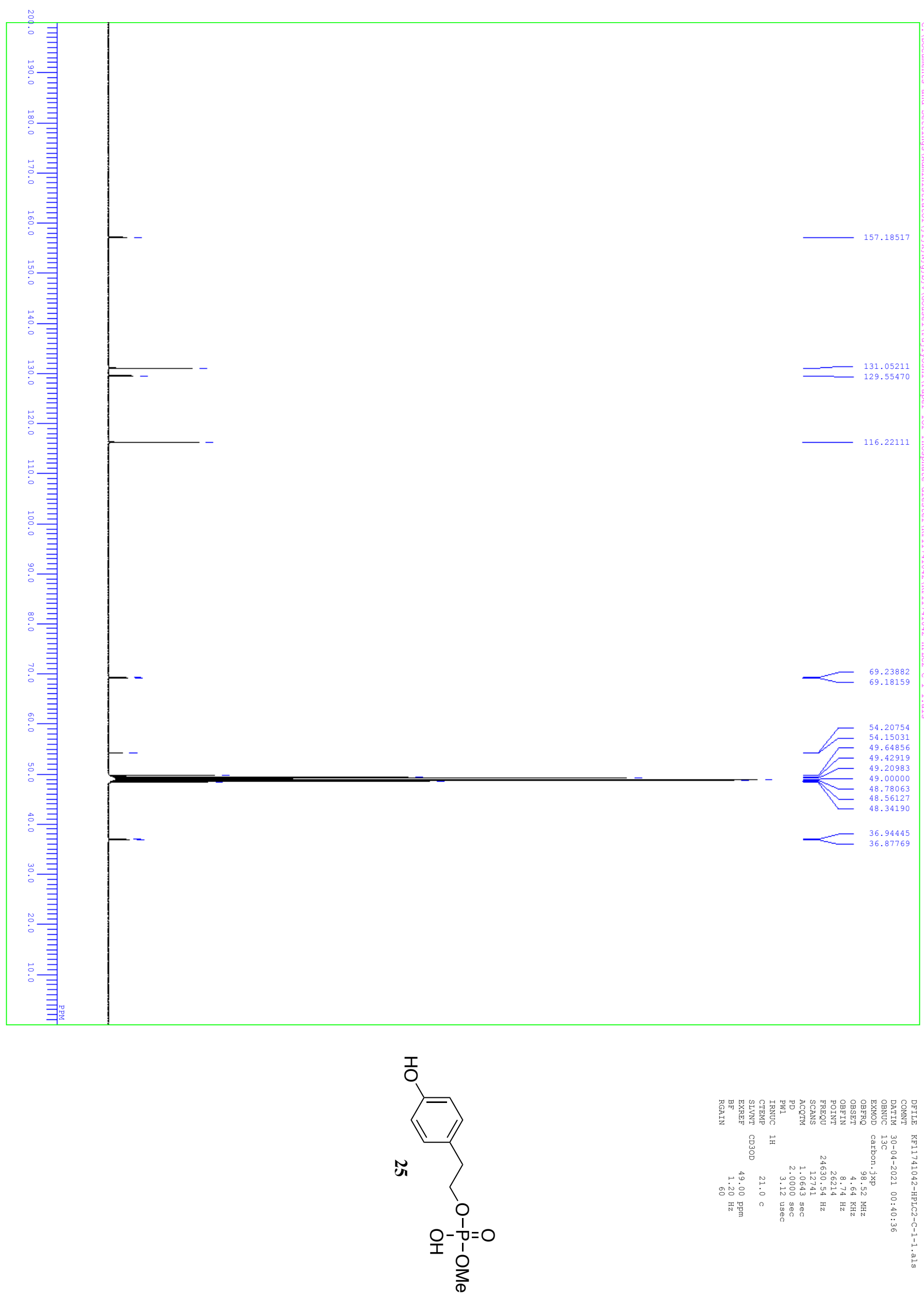

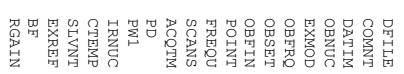

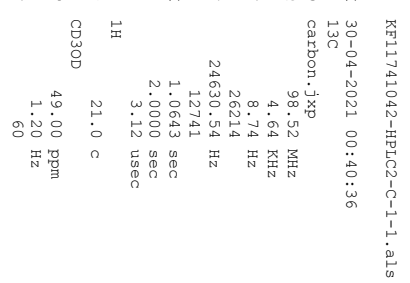



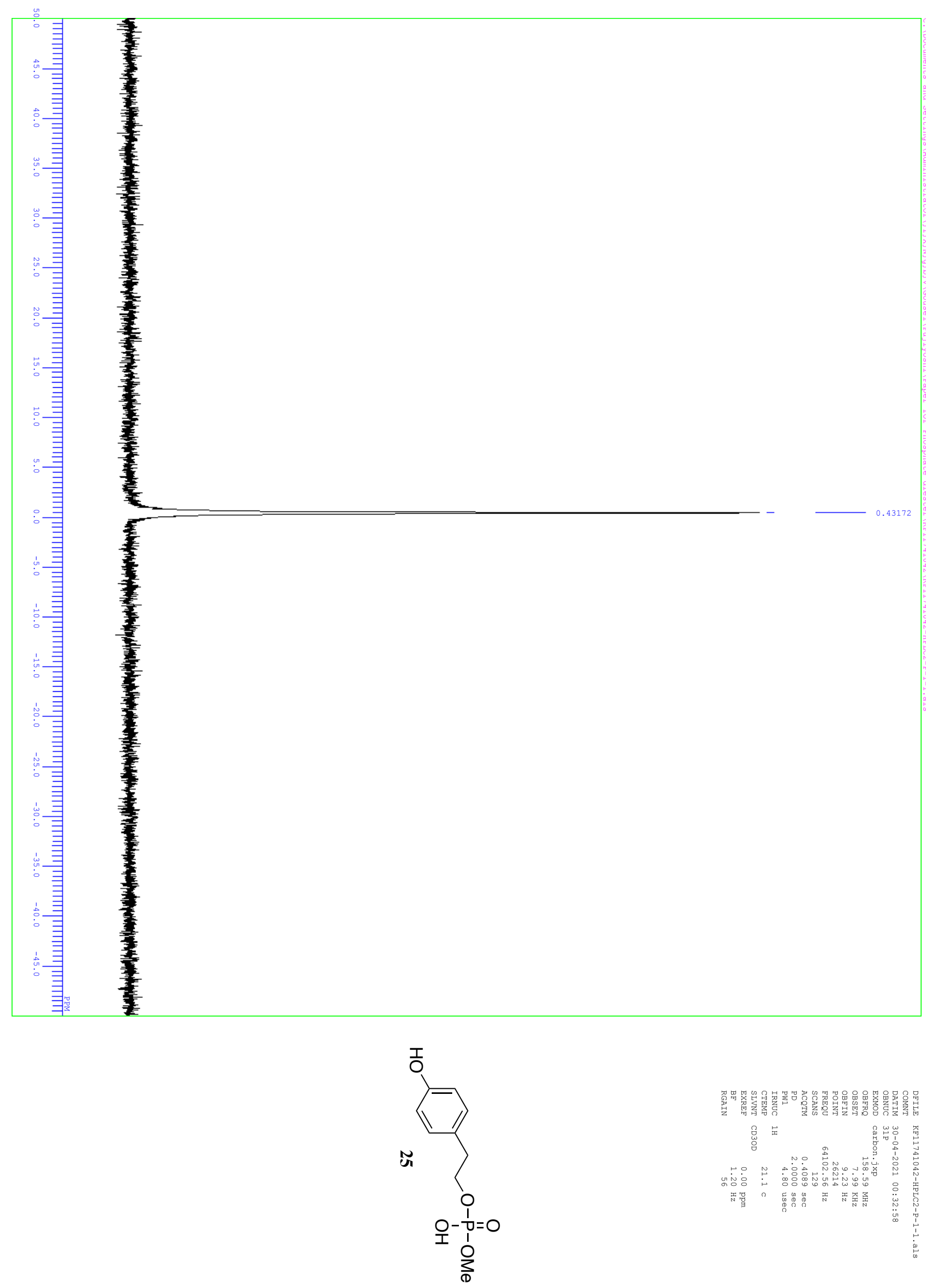

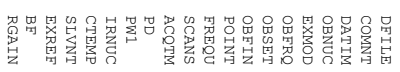

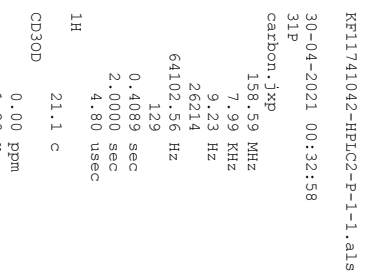



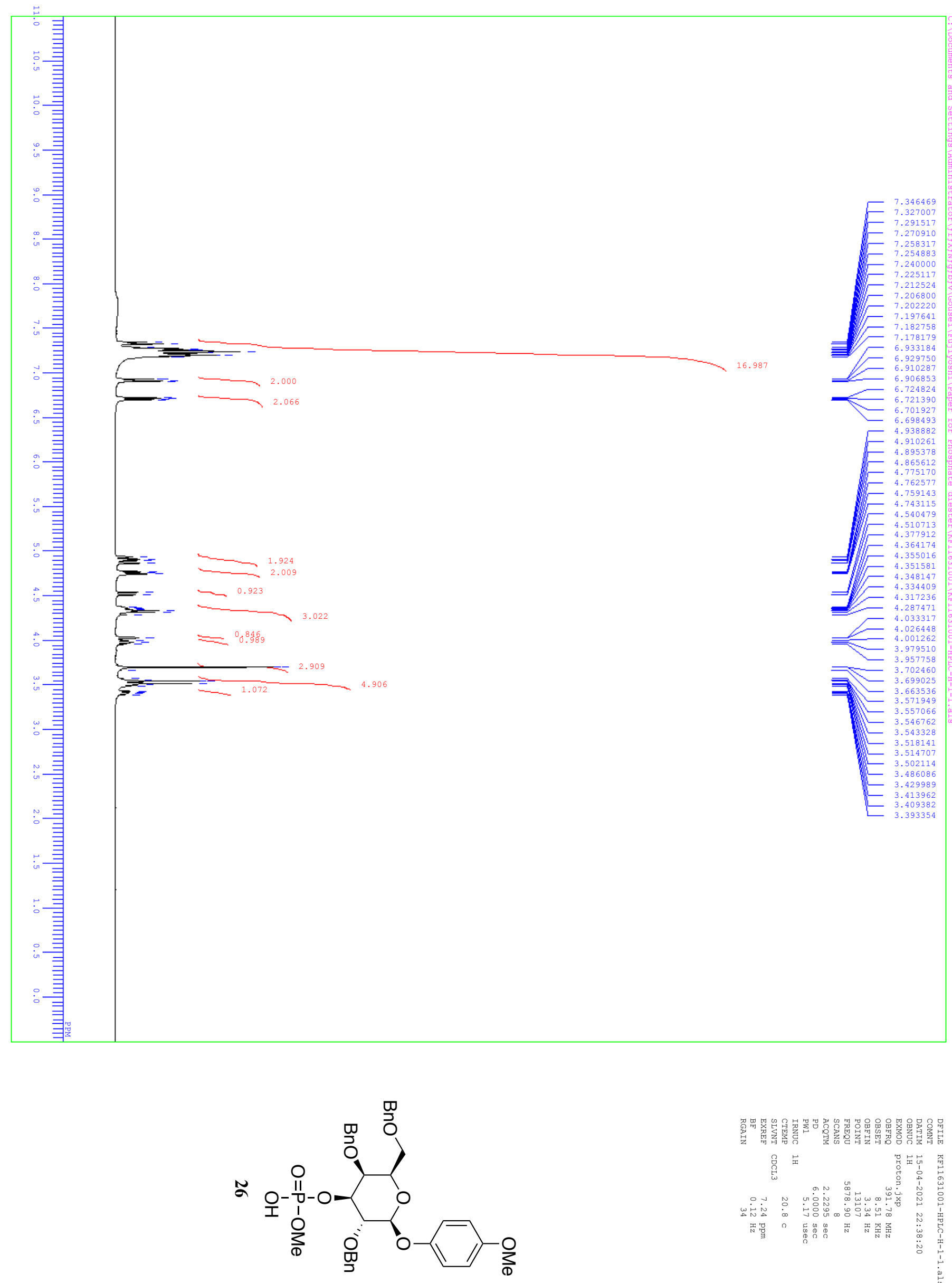

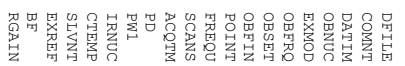
8 

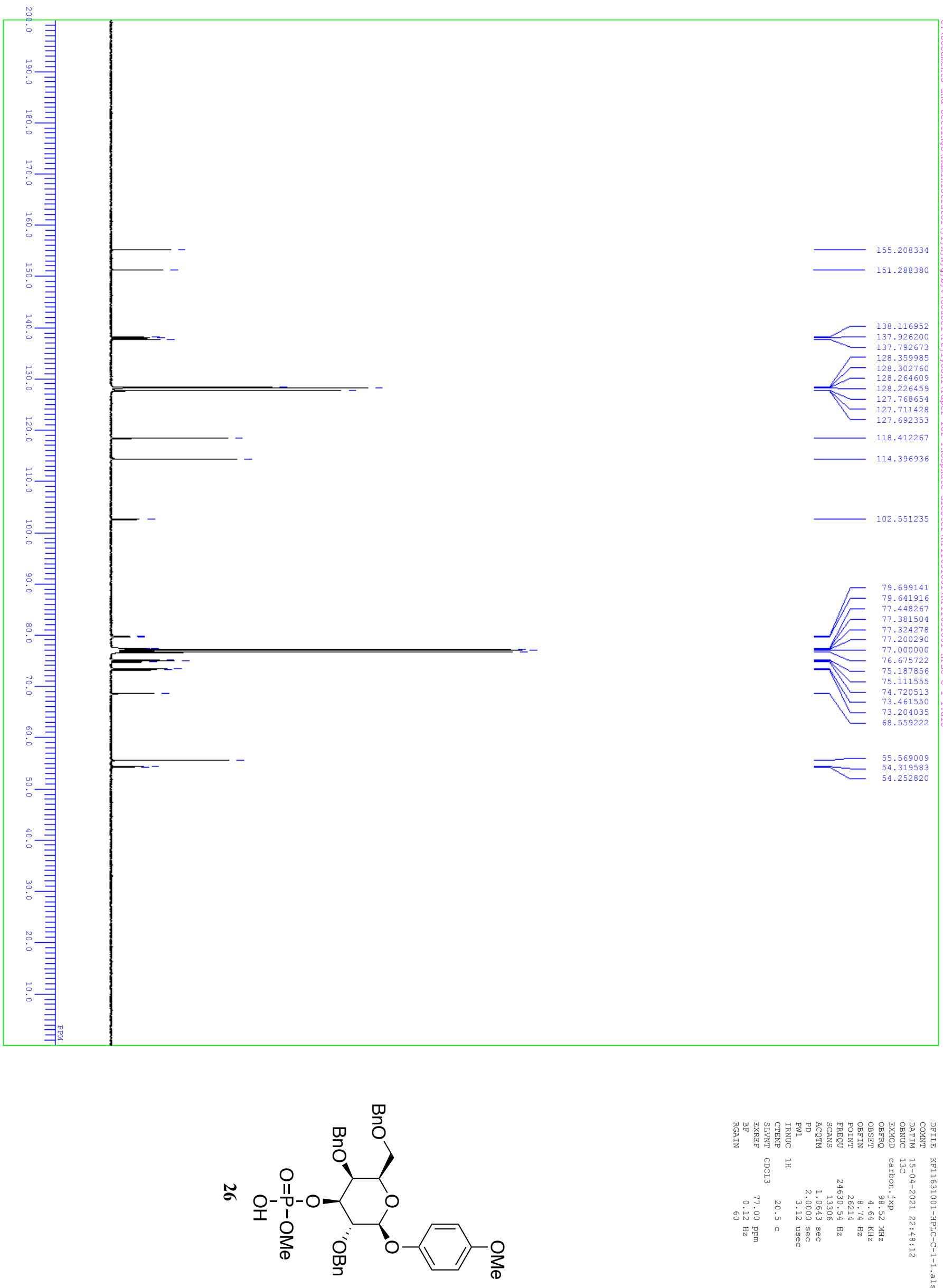

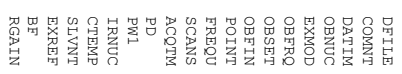

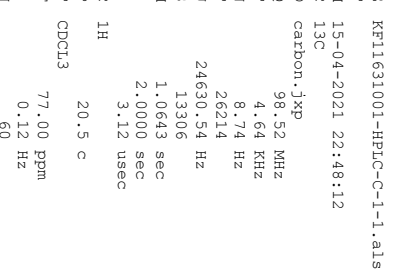



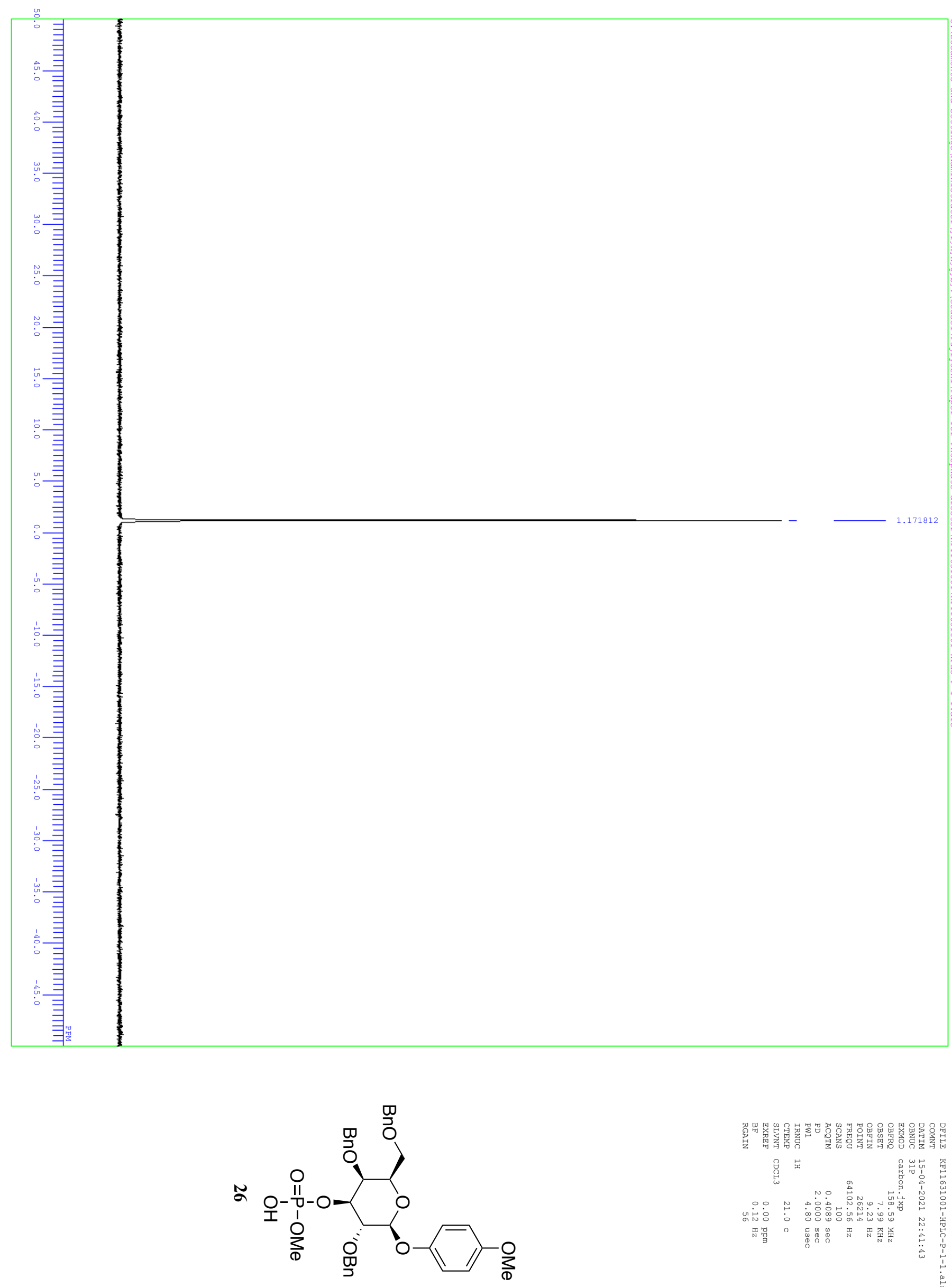

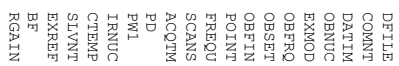

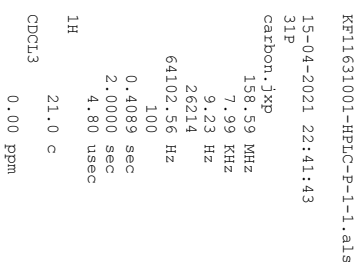



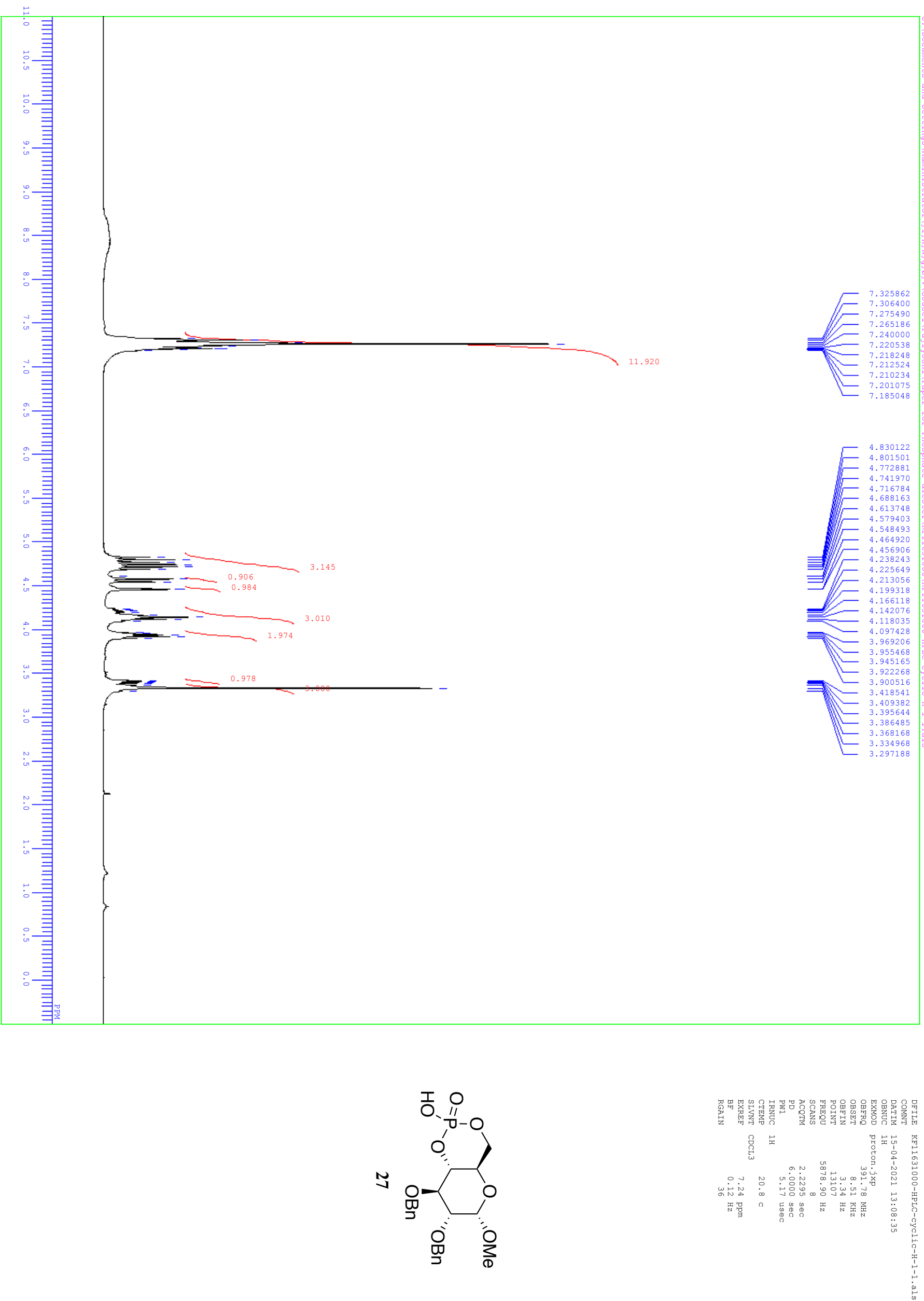

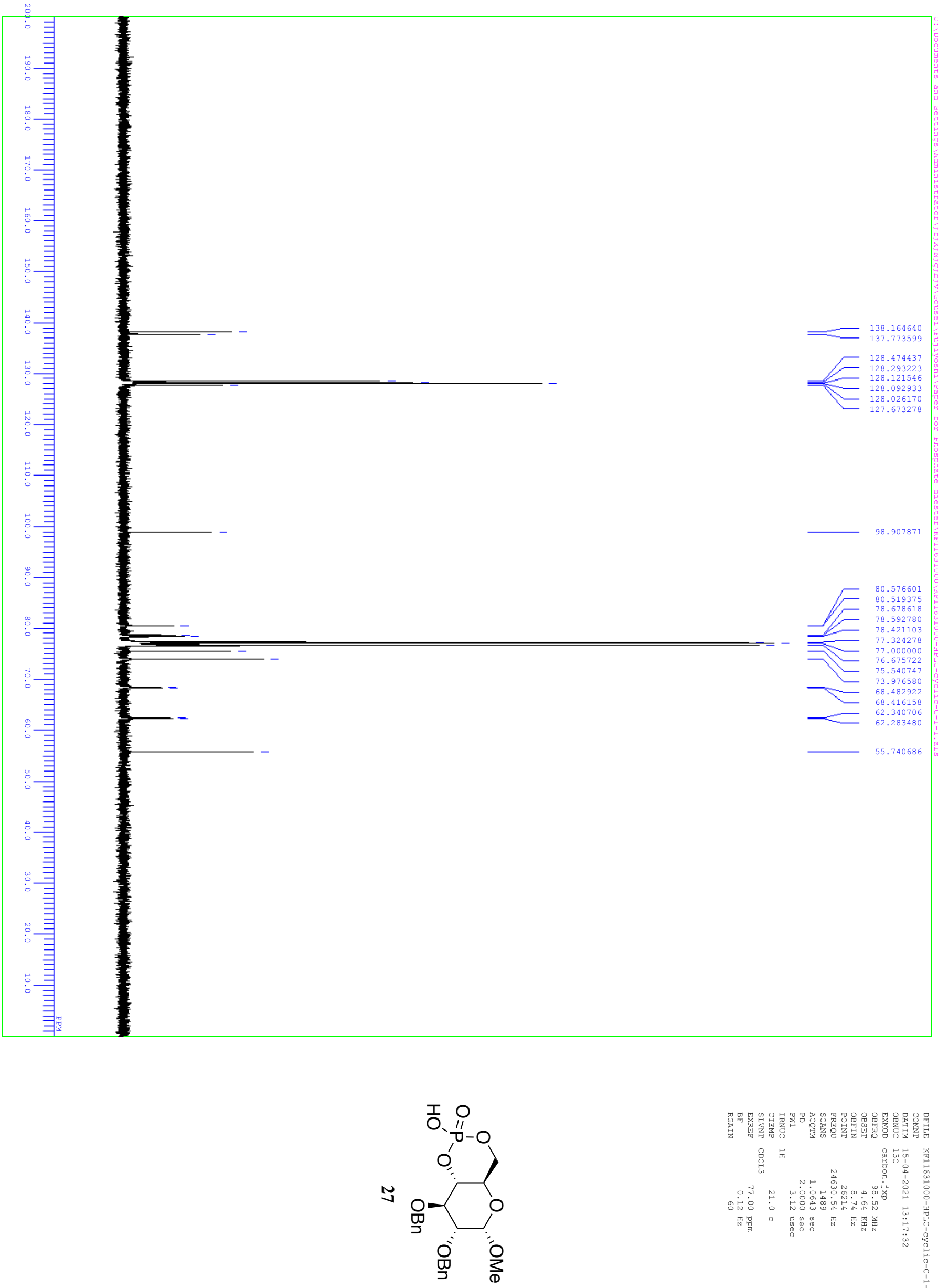

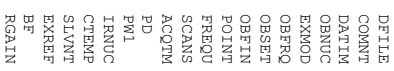

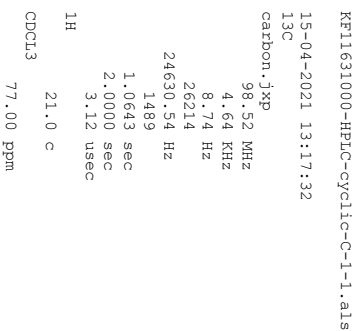



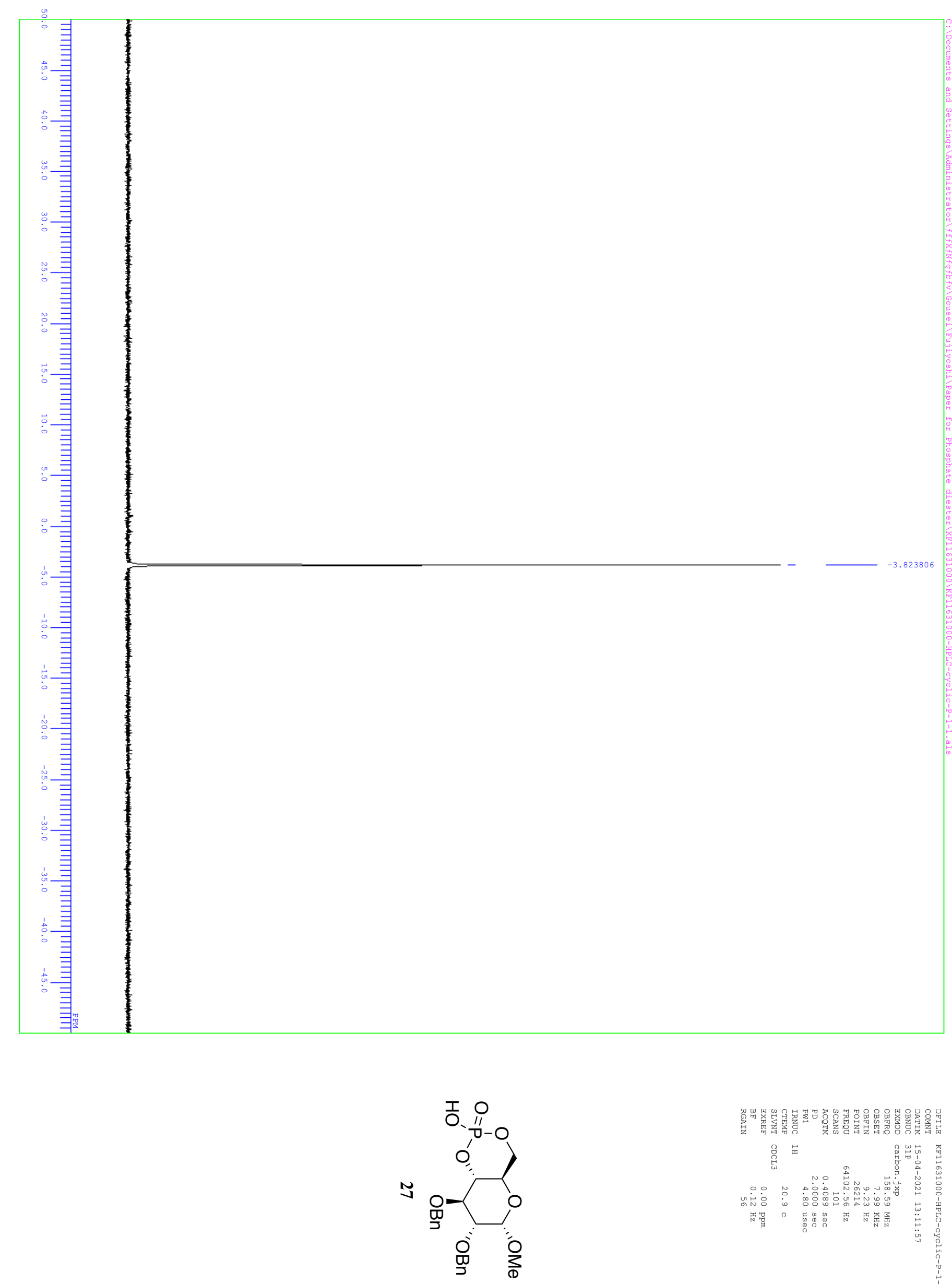

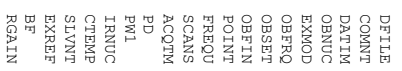
\& 的星高

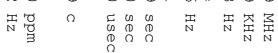



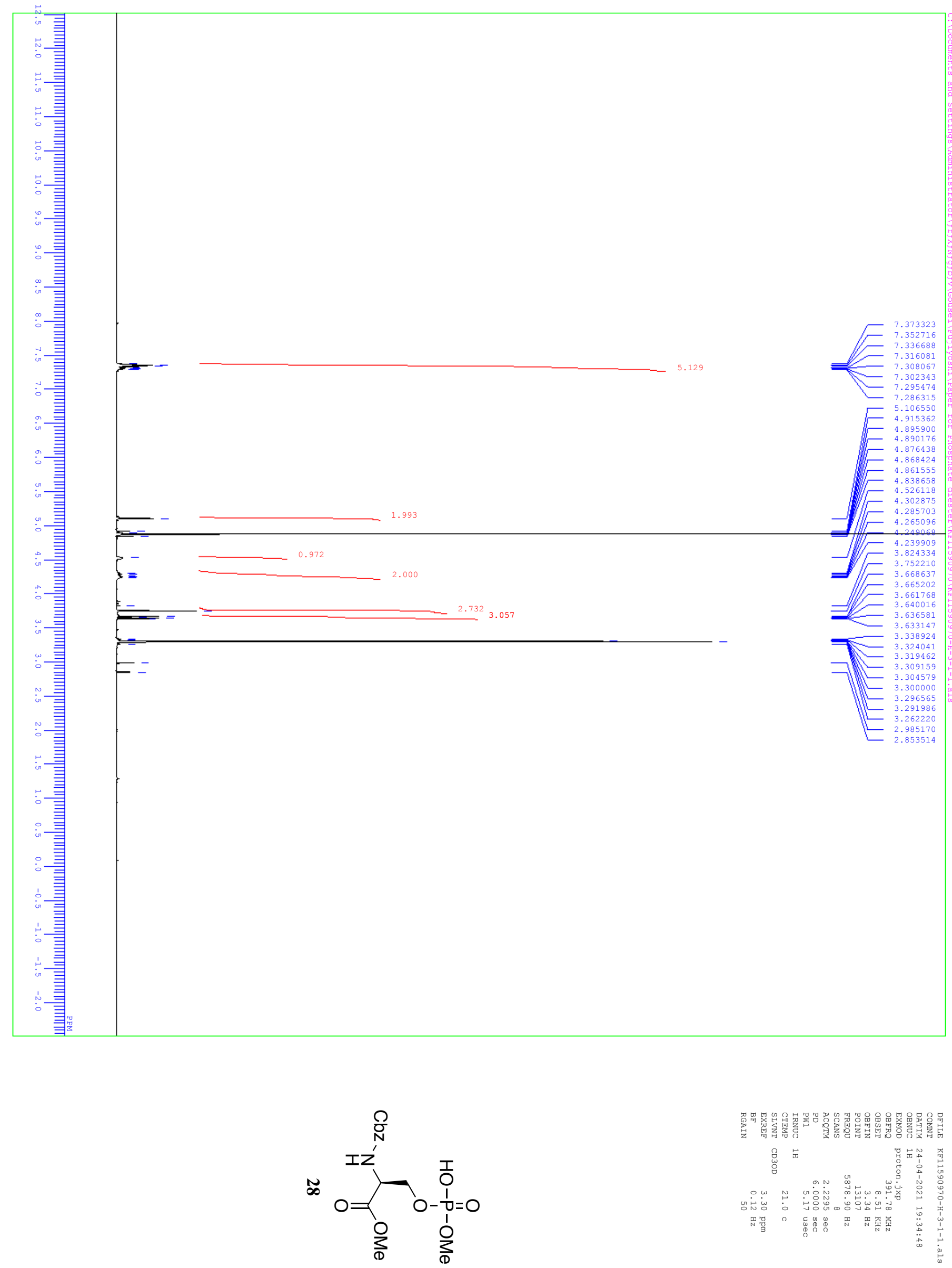

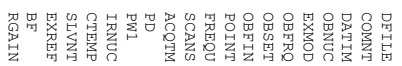

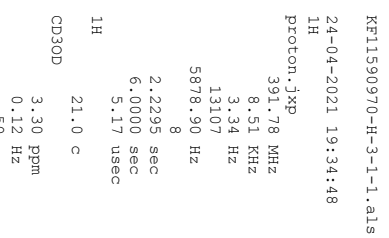



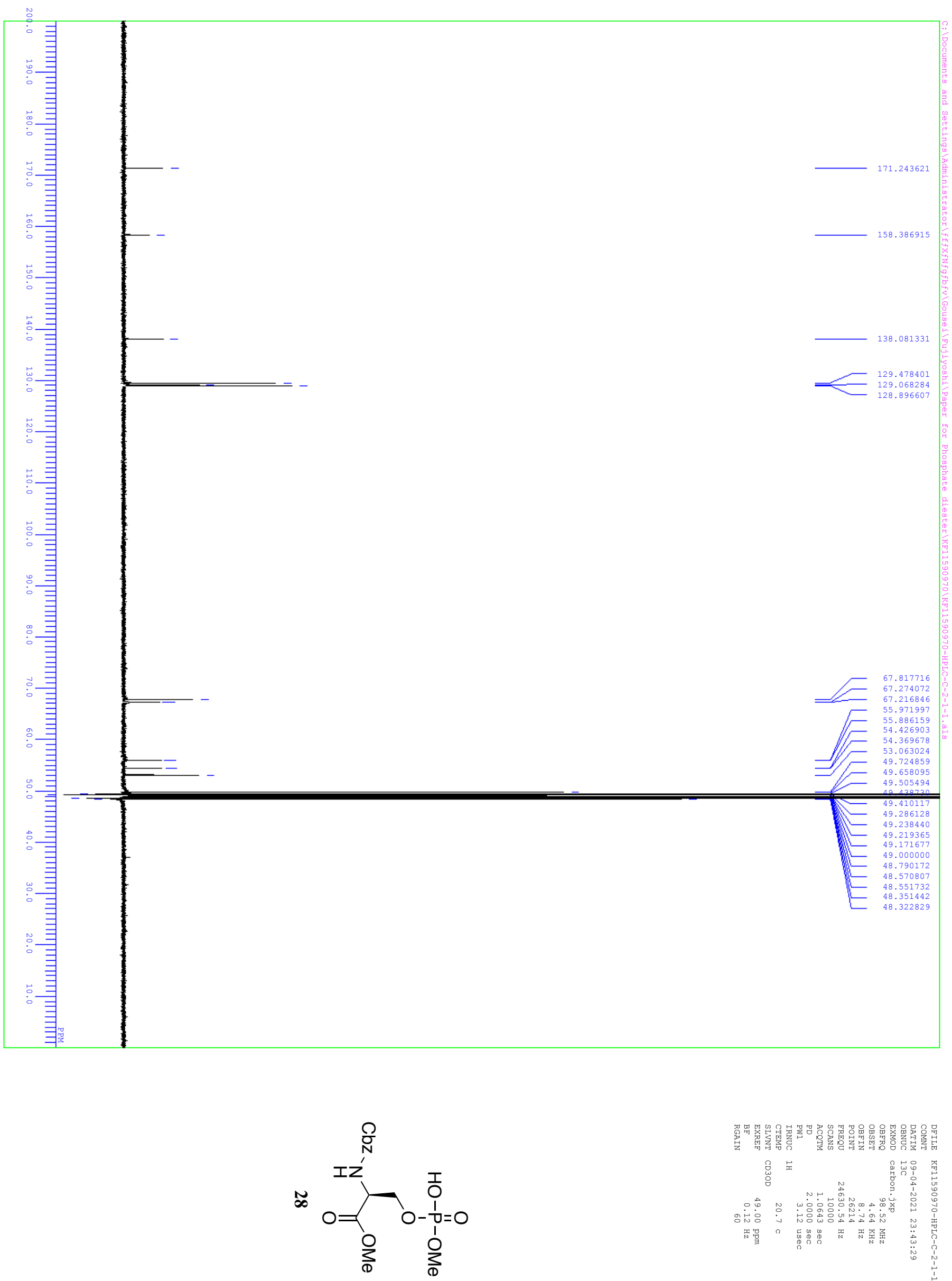

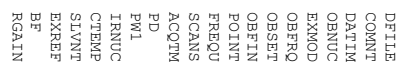

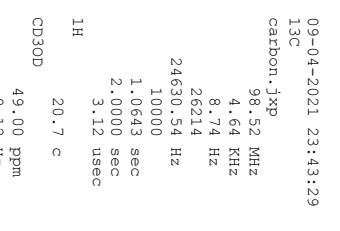



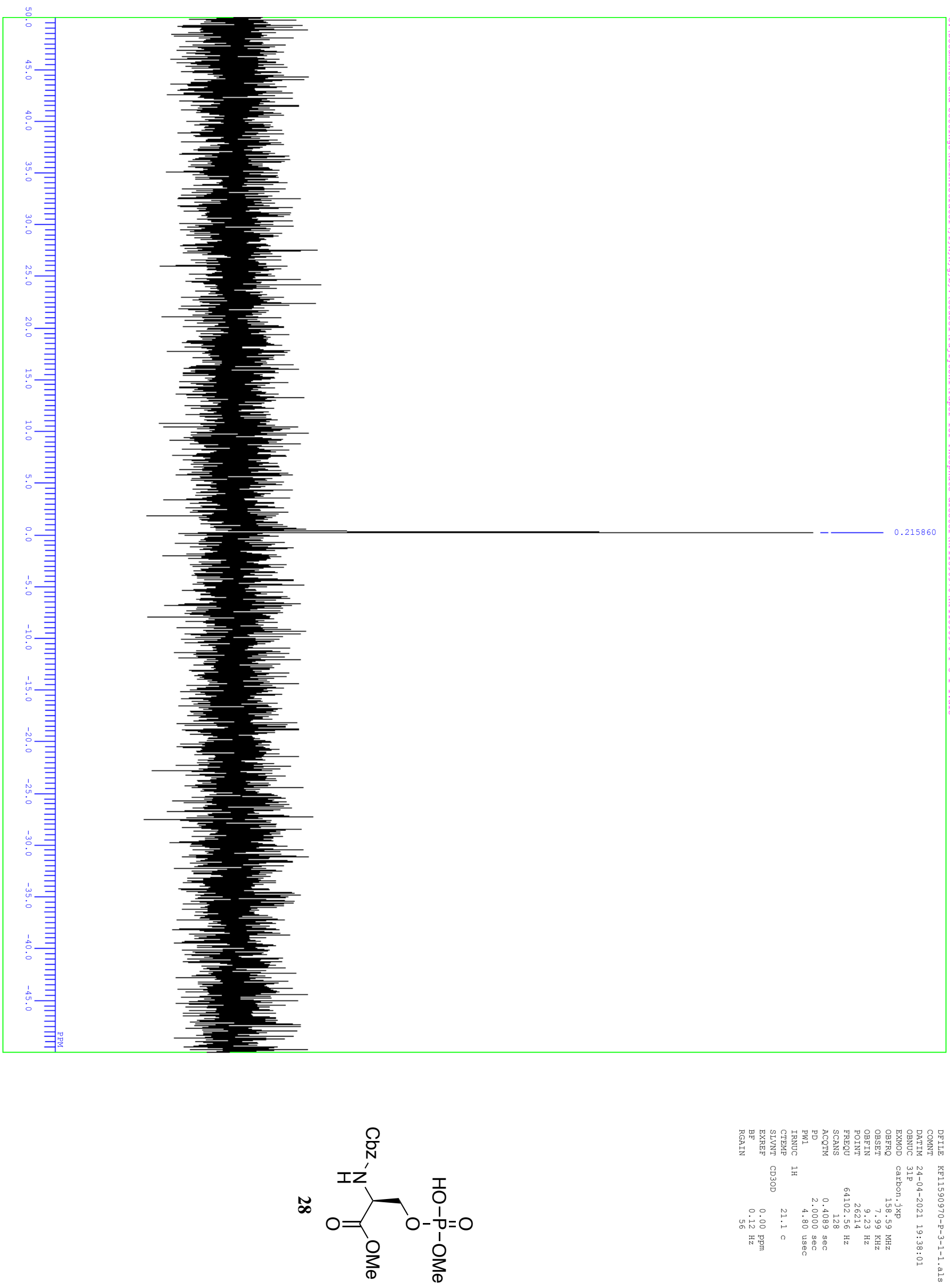

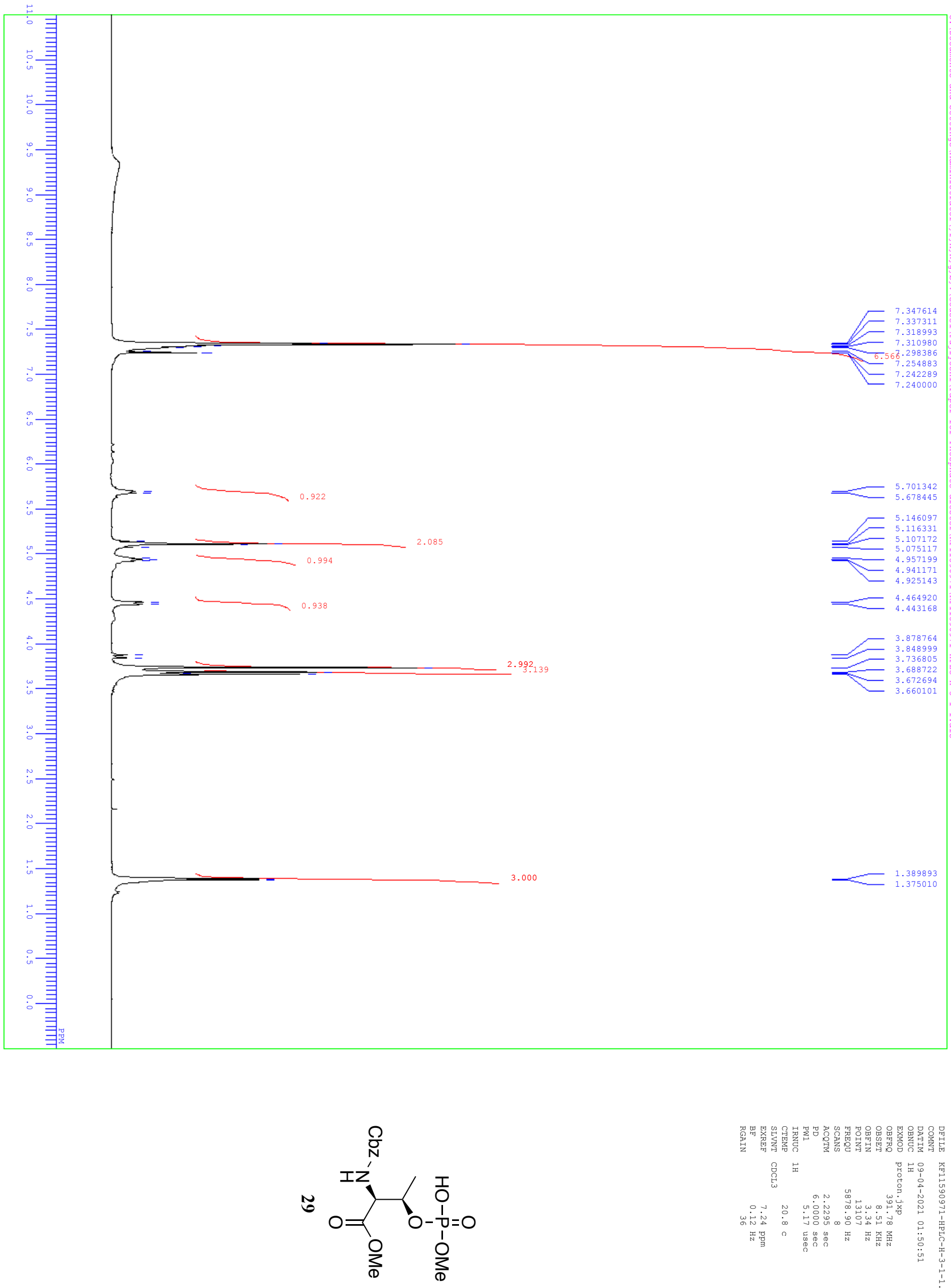

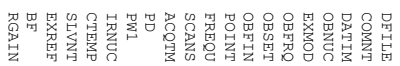

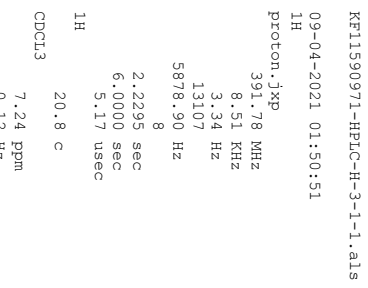



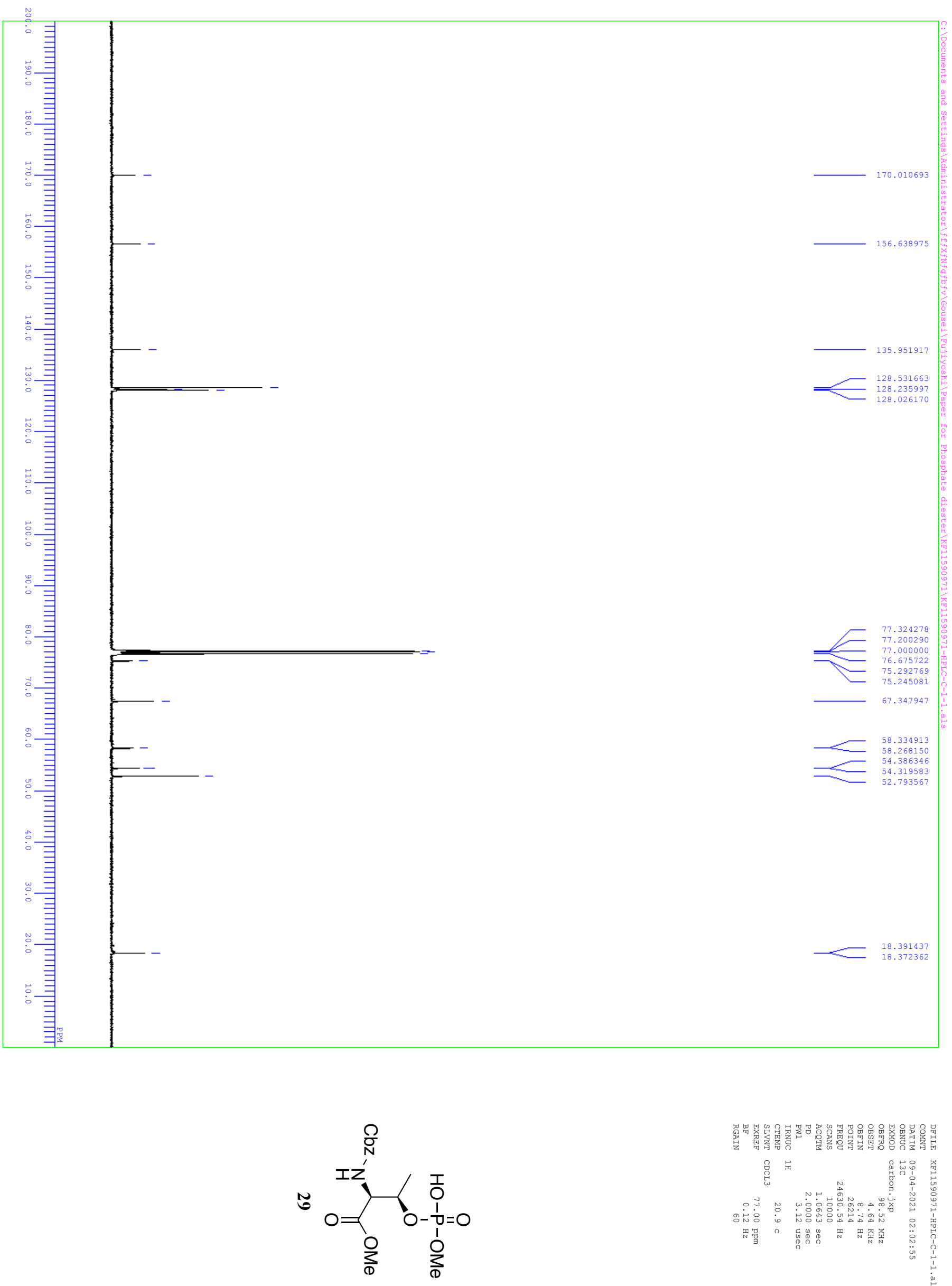

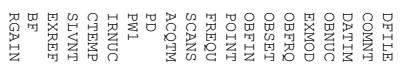

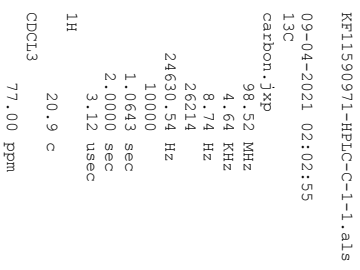



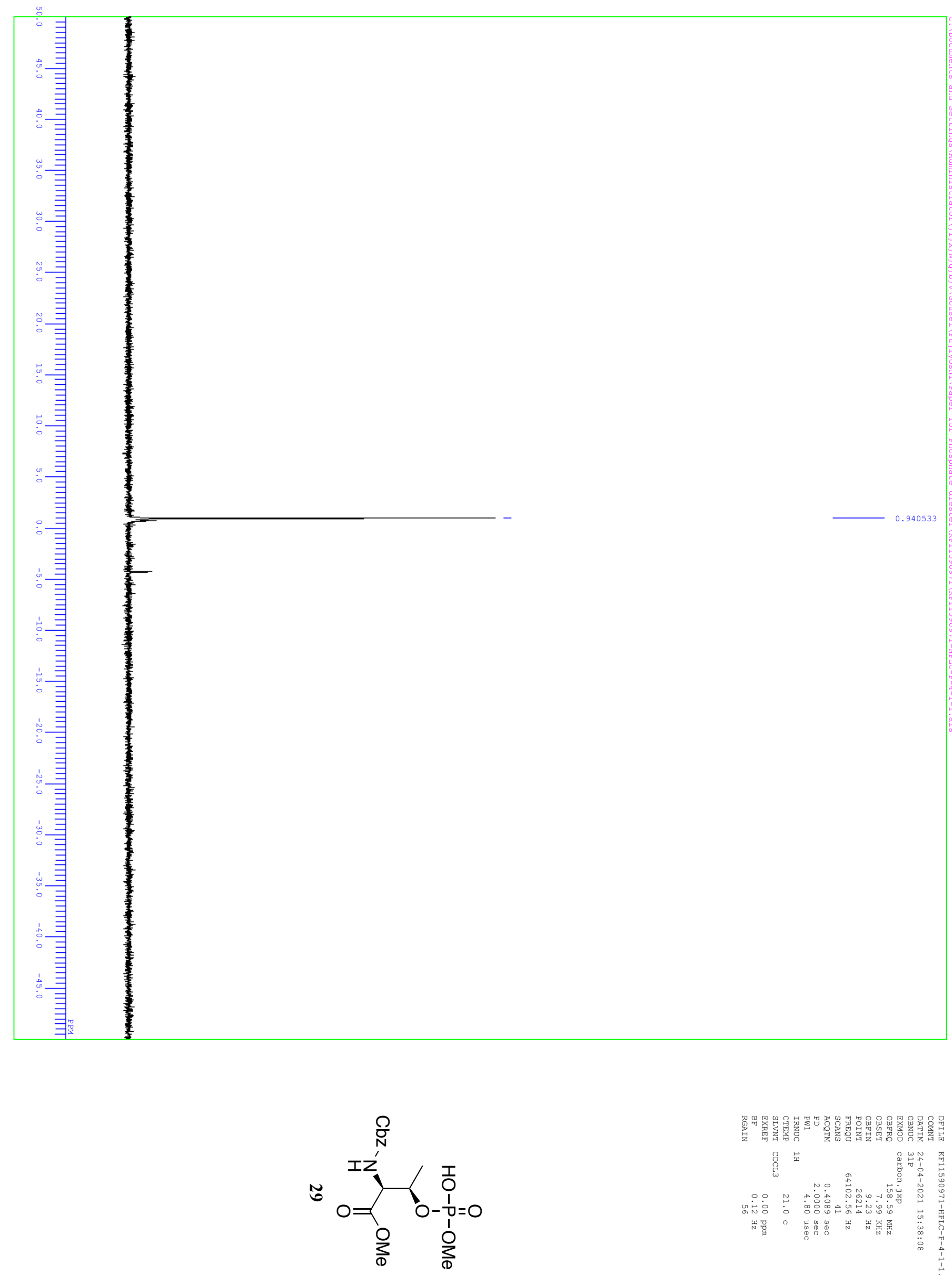

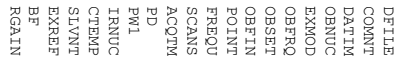

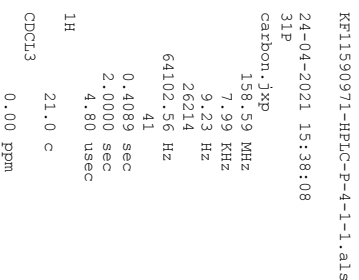



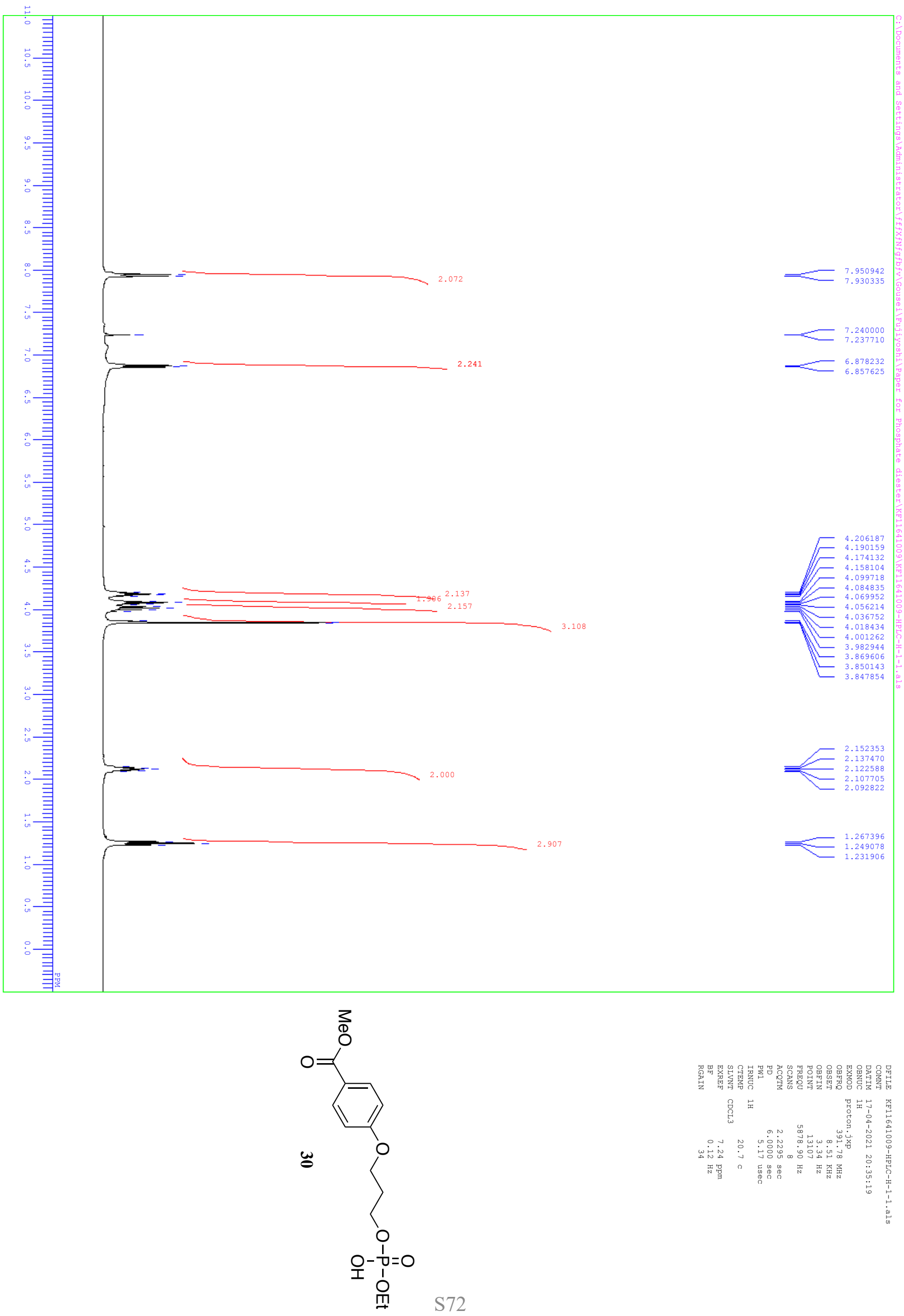

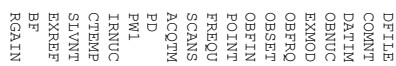
量星

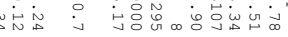

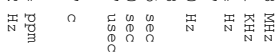



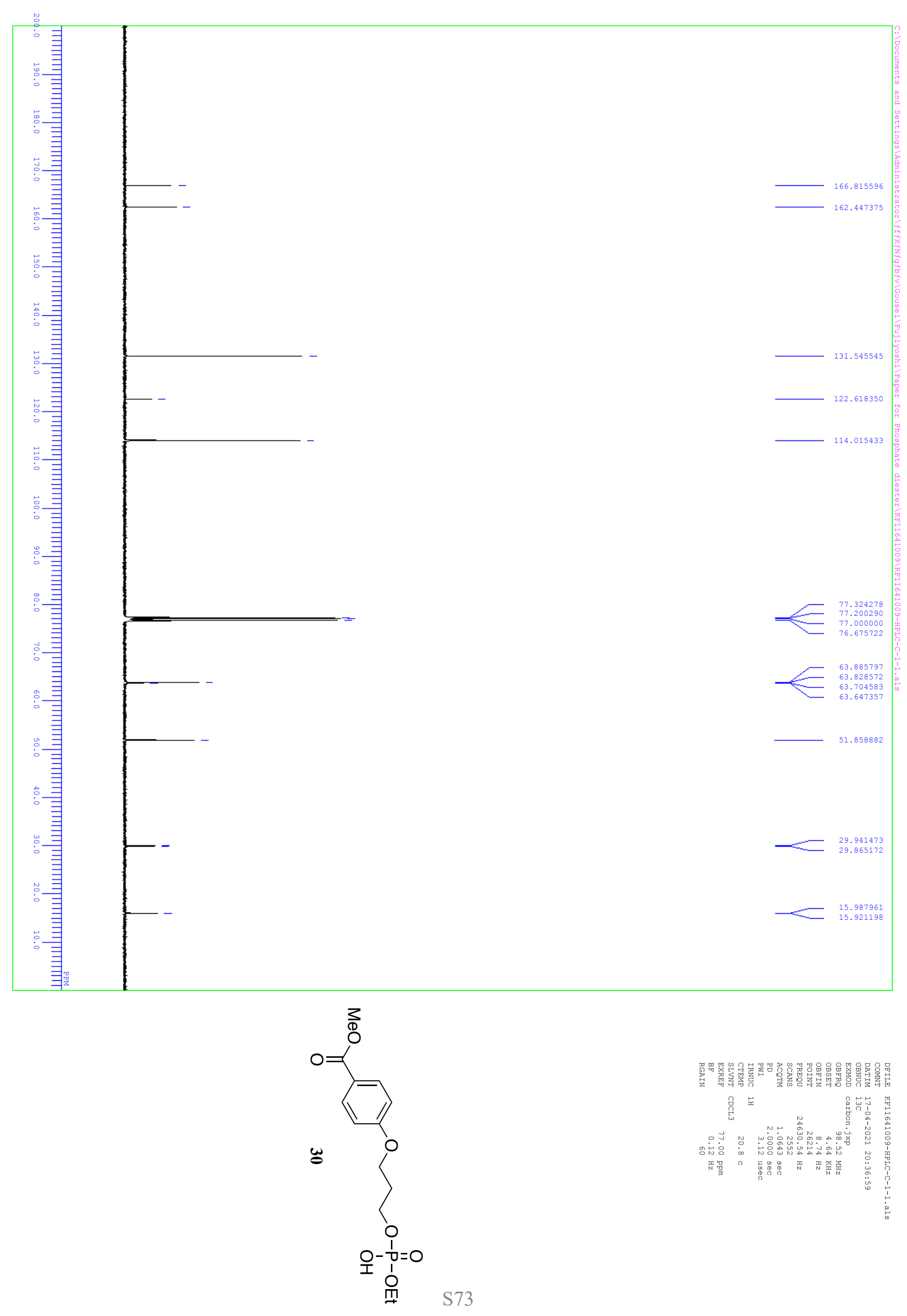


$$
F_{1}
$$



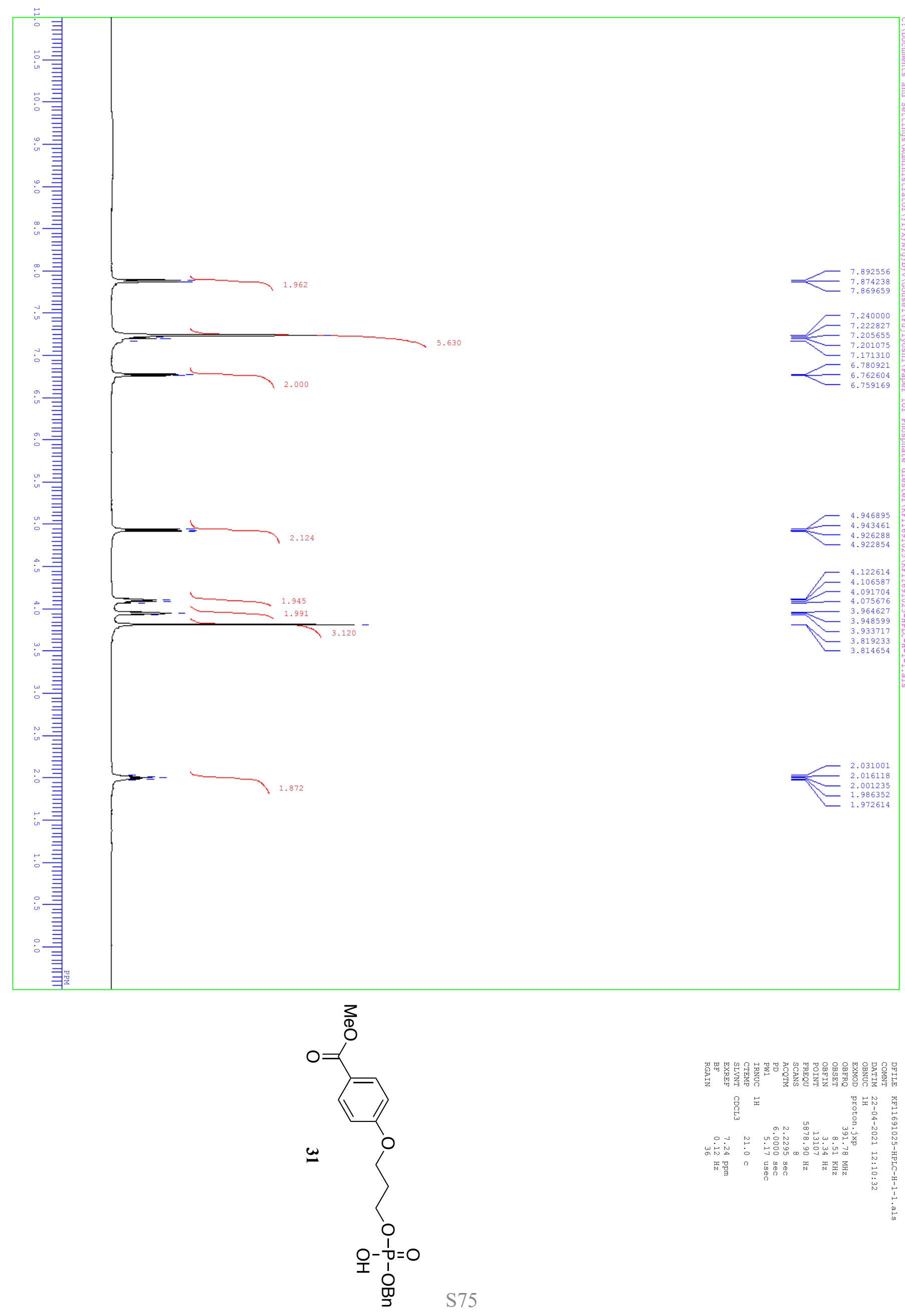

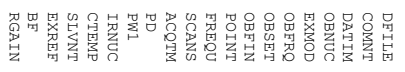

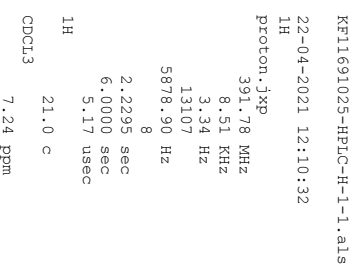



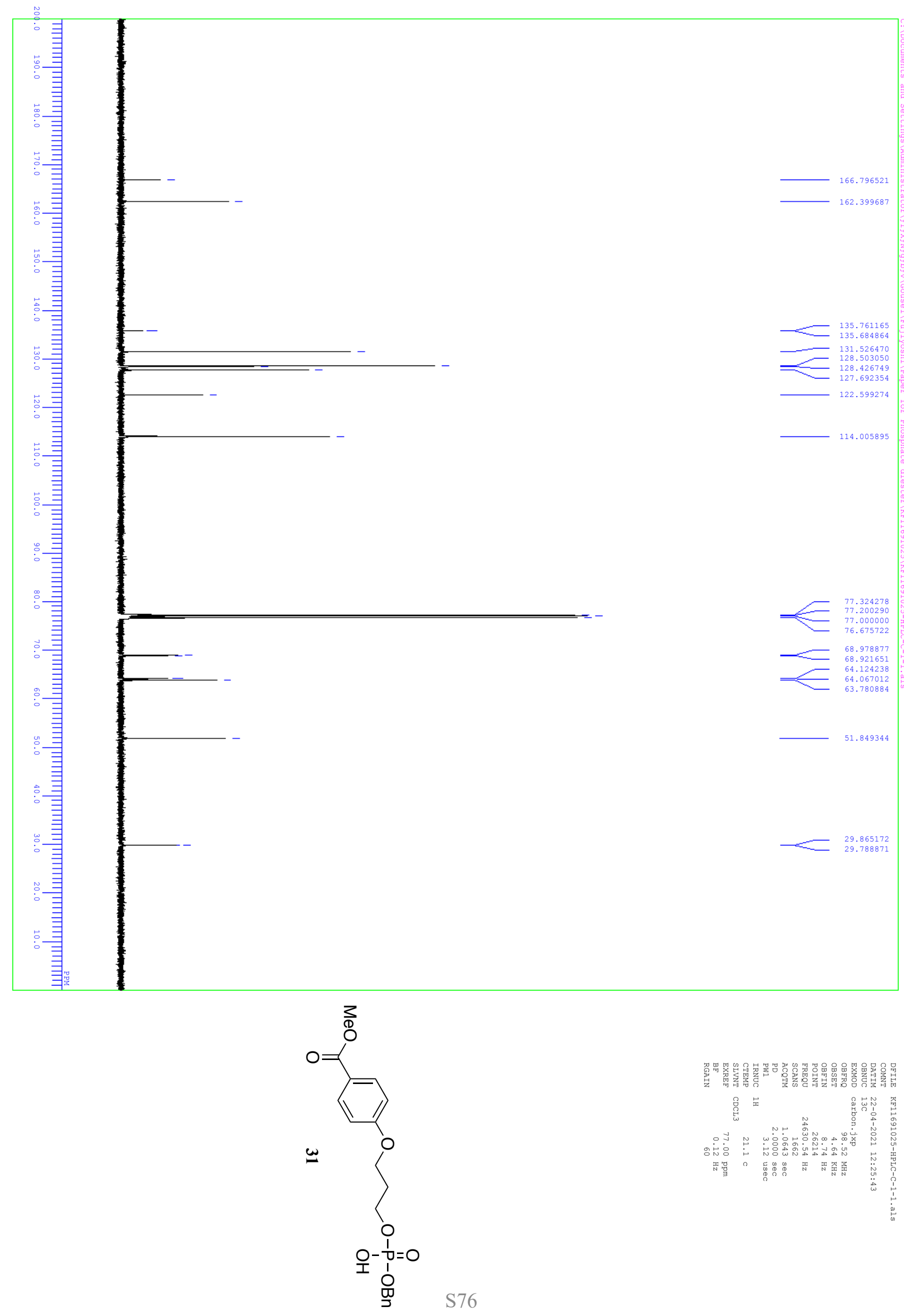

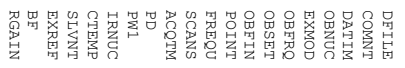

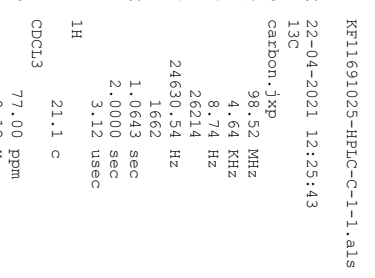


